\title{
Heterogeneity of Pestivirus Species in Asia
}

\author{
Massimo Giangaspero $^{1^{*}}\left(\mathbb{D}\right.$, Shu-Qin Zhang ${ }^{2}$, Claudio Apicella ${ }^{3}$ \\ ${ }^{1}$ Faculty of Veterinary Medicine, University of Teramo, Teramo, Italy \\ ${ }^{2}$ Key Laboratory of Special Animal Epidemic Disease, Ministry of Agriculture, Institute of Special Economic Animal and Plant \\ Sciences, Changchun, China \\ ${ }^{3}$ Directorate General of Veterinary Public Health, Food and Nutrition, Ministry of Health, Rome, Italy \\ Email: *giangasp@gmail.com
}

How to cite this paper: Giangaspero, M., Zhang, S.-Q. and Apicella, C. (2019) Heterogeneity of Pestivirus Species in Asia. Advances in Microbiology, 9, 266-342. https://doi.org/10.4236/aim.2019.93019

Received: January 25, 2019

Accepted: March 25, 2019

Published: March 28, 2019

Copyright (๑) 2019 by author(s) and Scientific Research Publishing Inc. This work is licensed under the Creative Commons Attribution International License (CC BY 4.0).

http://creativecommons.org/licenses/by/4.0/ (c) (i) Open Access

\begin{abstract}
Pestivirus are responsible for cosmopolitan diseases affecting cattle, pigs and other ruminants, presenting a wide range of clinical manifestations, with relevant impact on zootechnic production. Understanding genomic characteristic and virus taxonomy is fundamental in order to sustain control and prophylactic programs. Given the recent various studies reporting a relatively high number of new strains, in particular from Asian countries, in the present study, six hundred-fifty-one genomic sequences have been considered applying the palindromic nucleotide substitutions method for genotyping. Based on the secondary structure analysis of the 5' untranslated region of RNA, sequence characteristics among Asian genomic clusters within the different Pestivirus species suggested geographic segregation and occurrence of micro-evolutive steps in the genus evolutionary history. This aspect was particularly evident in atypical sequences originated from China or Turkey, indicating risk of diffusion by animals and products trade or contamination of biological products as bovine calf serum, with potential diagnostic and control difficulties.
\end{abstract}

\section{Keywords}

Asia, Genotypes, Pestivirus, Secondary Structure

\section{Introduction}

Species of the genus Pestivirus of the family Flaviviridae [1] are responsible for cosmopolitan disease affecting cattle pig and other ruminants, presenting a wide range of clinical manifestations, with relevant impact on zootechnic production. Different reports described atypical variants within the bovine viral diarrhoea virus type 1 (BVDV-1) (Pestivirus A), characterized by genetic traits different 
from most common previously reported strains, in particular from Asian countries [2]-[10]. Similarly, Asian atypical sequences have been reported in other Pestivirus species as BVDV-3 (Pestivirus $\mathrm{H}$ ) and Classical swine fever virus (CSFV) (Pestivirus C) [11] [12] [13]. Taking into account that a certain confusion in the nomenclature of the increasing number of Pestivirus genetic variants was evident and it is still a matter of concern, indicating that the need for harmonization [14] [15] [16], and the utility to have an alternative evaluation method are always interesting in any discipline, the palindromic nucleotide substitutions (PNS) method [17] [18] was proposed for an analytical procedure not limited to primary structure analysis but extended to secondary structure, focusing on strategic genomic sequences corresponding to the internal ribosome entry site (IRES), in the 5' untranslated region (UTR), responsible for translational, transcriptional and replication events in pestiviruses [19]. In order to evaluate more exhaustively the taxonomical segregation of the genus Pestivirus in Asian countries, the 5'-UTR genomic sequences of the BVDV-1, BVDV-2 (Pestivirus B), BVDV-3, Border Disease virus (BDV) (Pestivirus D) and CSFV species were analyzed and compared applying the PNS genotyping method, encompassing all Pestivirus species circulating in domestic animals in Asia and excluding the pestiviruses from chiropters.

\section{Material and Methods}

In order to determine genotypic variations in the BVDV-1, BVDV-2, BVDV-3, BDV and CSFV species, the 5'-UTR genomic region of six hundred-fifty-one Pestivirus strains, have been analyzed for numerical taxonomy. The virus nucleotide sequences, with different geographical origin, primarily of Asian origin, from different host species or contaminants of biological products, were obtained from the DDBL/EMBL/GenBank DNA database or provided by authors (Table 1). Strains from Europe and Americas have been considered as reference of genetic variants and complete comparison of strain sequences of BVDV-2, BVDV-3 and CSFV species. The majority of the tested virus sequences were originated from strains isolated from cattle (Bos taurus) from China (n 131). Other bovine strains originated from various other countries from Middle East, Indian subcontinent, Far East and Austral Asia (Turkey, Iran, Bangladesh, India, Thailand, Japan, South Korea, Australia and New Zealand) (n 69), and 89 were from Europe and Americas. Sixteen strains were isolated from buffalo (Bubalus bubalis) in China. Strains isolated from domestic Bactrian camels (Camelus bactrianus) and yaks (Bos grunniens) have also been reported from China (n 60). Seventeen strains were reported from Zebu (Bos indicus) in India, 116 strains were isolated from domestic and wild suids, pigs (Sus scrofa domesticus) and wild boar (Sus scrofa), 63 were from small ruminants, sheep (Ovis aries), goat (Capra hircus) and black goat (Capra aegagrus hircus). Three strains were from Alpine chamois (Rupicapra rupicapra), Pyrenean chamois (Rupicapra pyrenaica) and Reindeer (Rangifer tarandus), and 3 from Japanese serow (Capricornis crispus). A single 
Table 1. List of Pestivirus strains of BVDV-1 ( $\mathrm{n}=296)$, BVDV-2 $(\mathrm{n}=128)$, BVDV-3 $(\mathrm{n}=62)$, BDV $(\mathrm{n}=37)$, Pestivirus I ( $\mathrm{n}=2)$ and CSFV $(n=126)$ species evaluated according to palindromic secondary structure characteristics at the RNA 5'-UTR (PNS method). Nomenclature of identified genotypes is based on divergence in the genus. Clustering according to primary structure analysis by depositors is indicated under parenthesis. ND: not determined.

\begin{tabular}{|c|c|c|c|c|c|}
\hline Species/Genotype & Strain & Origin & Country & Accession & Reference \\
\hline BVDV-1a & 0192 & Contaminant & Japan & D31799 & [118] \\
\hline BVDV-1a & 12 & Cattle & Japan & D26051 & [117] \\
\hline BVDV-1a & 2L91 & Contaminant & Japan & D31800 & [118] \\
\hline BVDV-1a & A014 & Contaminant & Japan & D31801 & [118] \\
\hline BVDV-1a (A) & $\mathrm{B} 2-175 / \mathrm{AU}$ & Contaminant & Australia & JN967708 & [29] \\
\hline BVDV-1a (A) & BJ1308 & Cattle & China & KF925517 & [154] \\
\hline BVDV-1a & $\mathrm{C} 009 \mathrm{~T}$ & Contaminant & Japan & D31802 & [118] \\
\hline BVDV-1a (A) & E3-177/NZ & Contaminant & New Zealand & JN967722 & [29] \\
\hline BVDV-1a & FU411 & Contaminant & Japan & D31804 & [115] \\
\hline BVDV-1a (A) & H1-181/AU & Contaminant & Australia & JN967730 & [29] \\
\hline BVDV-1a & $\mathrm{H} 503$ & Contaminant & Japan & AB008841 & [112] \\
\hline BVDV-1a & HC725 & Contaminant & Japan & D31805 & [118] \\
\hline BVDV-1a & HE726 & Contaminant & Japan & D31806 & [118] \\
\hline BVDV-1a & HE728 & Contaminant & Japan & D31808 & [118] \\
\hline BVDV-1a & $\mathrm{HH}$ & Contaminant & Japan & D50818 & {$[30]$} \\
\hline BVDV-1a (A) & HN01 & Cattle & China & JX878887 & [5] \\
\hline BVDV-1a & IQ19A & Contaminant & Japan & D31812 & [118] \\
\hline BVDV-1a (A) & isolate 1 & Bactrian camel & China & JX276538 & [31] \\
\hline BVDV-1a (A) & isolate 2 & Bactrian camel & China & JX276539 & {$[31]$} \\
\hline BVDV-1a & $\mathrm{JE}$ & Contaminant & Japan & D26611 & [115] \\
\hline BVDV-1a & KQ25A & Contaminant & Japan & D31809 & [118] \\
\hline BVDV-1a & KQ25B & Contaminant & Japan & D31810 & [118] \\
\hline BVDV-1a $\quad(J)$ & KS86-1cp & Cattle & Japan & AB078952 & [138] \\
\hline BVDV-1a & L1305 & Cattle & New Zealand & AF026781 & Vilcek et al., unpublish \\
\hline BVDV-1a & LQ28A & Contaminant & Japan & D31811 & [118] \\
\hline BVDV-1a & MMR-K & Contaminant & Japan & D26050 & [117] \\
\hline BVDV-1a (A) & Nose & Cattle & Japan & AB019670 & {$[44]$} \\
\hline BVDV-1a (A) & SH1060 & Pig & China & JN248741 & {$[32]$} \\
\hline BVDV-1a & TK-87-2 & Cattle & Japan & AB019669 & [44] \\
\hline BVDV-1a (C) & Trangie Y546 & Cattle & Australia & AF049222 & Gu et al., unpublish \\
\hline BVDV-1a & TY CP/91 & Cattle & Japan & AB042670 & {$[90]$} \\
\hline BVDV-1.b1 (B) & BJ09_04 & Cattle & China & HQ116537 & Zhang et al., unpublish \\
\hline BVDV-1b1 & $\mathrm{CV}-1$ & Contaminant & Japan & D50815 & {$[30]$} \\
\hline BVDV-1b1 & FS720 & Contaminant & Japan & D31803 & [118] \\
\hline BVDV-1b1 & $\mathrm{HeLa}$ & Contaminant & Japan & D50819 & {$[30]$} \\
\hline BVDV-1.b1 (B) & JS-05059 & Cattle & China & KJ578849 & {$[2]$} \\
\hline BVDV-1b1 & MDBK & Contaminant & Japan & D50820 & {$[30]$} \\
\hline BVDV-1b1 & MDCK & Contaminant & Japan & D50821 & {$[30]$} \\
\hline BVDV-1b1 & MOLT-4 & Contaminant & Japan & D50822 & {$[30]$} \\
\hline BVDV-1b1 & Mumps & Contaminant & Japan & D26049 & [117] \\
\hline BVDV-1b1 & U937 & Contaminant & Japan & D50823 & {$[30]$} \\
\hline BVDV-1b1 & Vero & Contaminant & Japan & D50824 & {$[30]$} \\
\hline BVDV-1b1 & $\mathrm{Wi}-38$ & Contaminant & Japan & D50825 & {$[30]$} \\
\hline BVDV-1b1 & WiDr & Contaminant & Japan & D50826 & {$[30]$} \\
\hline BVDV-1b1 & Ind 446 & Cattle & India & AY279087 & [131] \\
\hline BVDV-1b1 & IndS1166 & Cattle & India & AY278460 & [131] \\
\hline BVDV-1b1 & IndS1168 & Cattle & India & AY279086 & [131] \\
\hline
\end{tabular}




\section{Continued}

\begin{tabular}{|c|c|c|c|c|c|}
\hline BVDV-1b1 & IndS1170 & Cattle & India & AY279526 & [131] \\
\hline BVDV-1b1 & IndS1171 & Cattle & India & AY279527 & {$[131]$} \\
\hline BVDV-1b1 & IndS1181 & Cattle & India & AY279528 & [131] \\
\hline BVDV-1b1 & IndS1222 & Cattle & India & AY278459 & [131] \\
\hline BVDV-1b1 & IndS1455 & Cattle & India & AY278461 & [131] \\
\hline BVDV-1b2 & IndMDV18697/12 & Zebu & India & KM201317 & [13] \\
\hline BVDV-1.b2 (B) & HB-0134 & Cattle & China & KJ578829 & [2] \\
\hline BVDV-1.b2 (B) & HB-0258 & Cattle & China & KJ578827 & [2] \\
\hline BVDV-1.b2 (B) & HB-0288 & Cattle & China & KJ578826 & [2] \\
\hline BVDV-1.b2 (B) & HB-060085 & Cattle & China & KJ578825 & [2] \\
\hline BVDV-1.b2 (B) & HB-0899 & Cattle & China & KJ578833 & [2] \\
\hline BVDV-1.b2 (B) & HB-090219 & Cattle & China & KJ578828 & [2] \\
\hline BVDV-1b2 (B) & isolate 12 & Bactrian camel & China & JX276549 & [31] \\
\hline BVDV-1b2 & KA-91 & Cattle & Japan & AB019684 & [44] \\
\hline BVDV-1.b2 (B) & LN311-15 & Cattle & China & KJ578799 & [2] \\
\hline BVDV-1.b2 (B) & LN311-17 & Cattle & China & KJ578798 & [2] \\
\hline BVDV-1.b2 (B) & LN311-25 & Cattle & China & KJ578797 & [2] \\
\hline BVDV-1.b2 (B) & LN311-27 & Cattle & China & KJ578796 & [2] \\
\hline BVDV-1.b2 (B) & LN311-34 & Cattle & China & KJ578795 & [2] \\
\hline BVDV-1.b2 (B) & LN313-15 & Cattle & China & KJ578800 & {$[2]$} \\
\hline BVDV-1.b2 (B) & LN314-19 & Cattle & China & KJ578801 & [2] \\
\hline BVDV-1.b2 (B) & QHHY-21 & Yak & China & KJ578902 & [2] \\
\hline BVDV-1.b2 (B) & QHMY-N5 & Yak & China & KJ578900 & [2] \\
\hline BVDV-1.b2 (B) & QHMY-N6 & Yak & China & KJ578901 & [2] \\
\hline BVDV-1.b2 (B) & QHQL-126 & Yak & China & KJ578880 & [2] \\
\hline BVDV-1.b2 (B) & QHQL-219 & Yak & China & KJ578890 & [2] \\
\hline BVDV-1.b2 (B) & QHQL-271 & Yak & China & KJ578891 & [2] \\
\hline BVDV-1.b2 (B) & QHQL-292 & Yak & China & KJ578883 & [2] \\
\hline BVDV-1.b2 (B) & QHQL-295 & Yak & China & KJ578886 & [2] \\
\hline BVDV-1.b2 (B) & QHQL-297 & Yak & China & KJ578892 & [2] \\
\hline BVDV-1.b2 (B) & QHQL-299 & Yak & China & KJ578893 & [2] \\
\hline BVDV-1.b2 (B) & QHQL-311 & Yak & China & KJ578873 & [2] \\
\hline BVDV-1.b2 (B) & QHQL-313 & Yak & China & KJ578874 & [2] \\
\hline BVDV-1.b2 (B) & QHQL-321 & Yak & China & KJ578877 & [2] \\
\hline BVDV-1.b2 (B) & QHQL-328 & Yak & China & KJ578875 & [2] \\
\hline BVDV-1.b2 (B) & QHQL-336 & Yak & China & KJ578887 & [2] \\
\hline BVDV-1.b2 (B) & QHQL-337 & Yak & China & KJ578876 & [2] \\
\hline BVDV-1.b2 (B) & QHQL-340 & Yak & China & KJ578878 & [2] \\
\hline BVDV-1.b2 (B) & QHQL-345 & Yak & China & KJ578879 & [2] \\
\hline BVDV-1.b2 (B) & QHQL-385 & Yak & China & KJ578888 & [2] \\
\hline BVDV-1.b2 (B) & QHQL-405 & Yak & China & KJ578889 & [2] \\
\hline BVDV-1.b2 (B) & QHTJ-17 & Yak & China & KJ578896 & {$[2]$} \\
\hline BVDV-1.b2 (B) & QHTJ-291 & Yak & China & KJ578894 & [2] \\
\hline BVDV-1.b2 (B) & QHTJ-L332 & Yak & China & KJ578895 & [2] \\
\hline BVDV-1.b2 (B) & QHTJ-N303868 & Yak & China & KJ578897 & [2] \\
\hline BVDV-1.b2 (B) & QHTJ-N304810 & Yak & China & KJ578898 & [2] \\
\hline BVDV-1.b2 (B) & XZ-117 & Yak & China & KJ578904 & [2] \\
\hline BVDV-1.b2 (B) & $\mathrm{XZ}-70$ & Yak & China & KJ578911 & [2] \\
\hline BVDV-1.3 (D) & 10JJ-SKR & Cattle & South Korea & KC757383 & [123] \\
\hline BVDV-1.3 (D) & B1-1/AU & Contaminant & Australia & JN967745 & [29] \\
\hline BVDV-1.3 (D) & BJ1023 & Cattle & China & KF925509 & [154] \\
\hline
\end{tabular}




\section{Continued}

\begin{tabular}{|c|c|c|c|c|c|}
\hline BVDV-1.3 (D) & BJ1120 & Cattle & China & KF925510 & [154] \\
\hline BVDV-1.3 (D) & BJ1201 & Cattle & China & KF925513 & [154] \\
\hline BVDV-1.3 & $\mathrm{BRU}^{*} 0615$ & Contaminant & Japan & AB008837 & [112] \\
\hline BVDV-1.3 (D) & cell-con-1 & Cattle & China & KC695816 & Gao et al., unpublish \\
\hline BVDV-1.3 (D) & IR-Isfahan-10 & Cattle & Iran & LC053999 & Shapouri et al., unpublish \\
\hline BVDV-1.3 (D) & OK1(CA)NCP03 & Cattle & Japan & AB359927 & [28] \\
\hline BVDV-1.3 & Kamoshika-3 & Serow & Japan & AB259693 & [63] \\
\hline BVDV-1.3 & Kamoshika-22 & Serow & Japan & AB259694 & [63] \\
\hline BVDV-1.3 & Kamoshika-29 & Serow & Japan & AB259695 & [63] \\
\hline BVDV-1.4 & $438 / 02$ & Cattle & Spain & AY159540 & [70] \\
\hline BVDV-1.5 & $23-15$ & Cattle & UK & AF298059 & [165] \\
\hline BVDV-1.6 (N) & $06 z 71$ & Cattle & South Korea & DQ973181 & Yang and Kweon, unpublish \\
\hline BVDV-1.6 (N) & $06 z 127$ & Cattle & South Korea & DQ973182 & Yang and Kweon, unpublish \\
\hline BVDV-1.6 (N) & KB01 & Cattle & South Korea & GQ495676 & Oem et al., unpublish \\
\hline BVDV-1.6 (M) & S133 & Cattle & China & KJ690689 & {$[8]$} \\
\hline BVDV-1.6 (N) & Shitara/02/06 & Cattle & Japan & LC089876 & [91] \\
\hline BVDV-1.6 (N) & so $\mathrm{CP} / 75$ & Cattle & Japan & AB042661 & [90] \\
\hline BVDV-1.6 (M) & TY05 & Cattle & China & GU120242 & [7] \\
\hline BVDV-1.7 (O) & AQGN96BI5 & Cattle & Japan & AB300691 & [168] \\
\hline BVDV-1.7 (M) & BJ09_24 & Cattle & China & HQ116550 & [8] \\
\hline BVDV-1.7 (O) & IS25CP/01 & Cattle & Japan & AB359931 & [28] \\
\hline BVDV-1.7 (O) & IS26/01ncp & Cattle & Japan & AB359932 & {$[28]$} \\
\hline BVDV-1.7 (M) & isolate 9 & Bactrian camel & China & JX276546 & [31] \\
\hline BVDV-1.7 (O) & JS10116 & Pig & China & JN248734 & [32] \\
\hline BVDV-1.7 (M) & S121 & Cattle & China & KF006960 & [8] \\
\hline BVDV-1.7 (M) & S43 & Cattle & China & KF006959 & [8] \\
\hline BVDV-1.8 & A1-114/AU & Contaminant & Australia & JN967700 & [29] \\
\hline BVDV-1.8 (C) & AQMZ02AI21/2 & Cattle & Australia & AB300687 & [168] \\
\hline BVDV-1.8 (C) & Bega & Cattle & Australia & AF049221 & Mackintosh et al., unpublish \\
\hline BVDV-1.8 (C) & Bega-like & Cattle & Australia & KF896608 & Gao et al., unpublish \\
\hline BVDV-1.8 & CRFK & Contaminant & Japan & D50814 & {$[30]$} \\
\hline BVDV-1.8 (C) & isolate 3 & Bactrian camel & China & JX276540 & [31] \\
\hline BVDV-1.8 (C) & isolate 4 & Bactrian camel & China & JX276541 & [31] \\
\hline BVDV-1.8 (C) & Letuyi & Cattle & China & EU159701 & [9] \\
\hline BVDV-1.8 (C) & Manasi & Cattle & China & EU159702 & [9] \\
\hline BVDV-1.8 (C) & S183 & Contaminant & China & KF006968 & [8] \\
\hline BVDV-1.8 (C) & Shitara/01/05 & Cattle & Japan & AB359926 & [28] \\
\hline BVDV-1.8 (C) & Shihezi 148 & Cattle & China & EU159700 & [9] \\
\hline BVDV-1.9 & KM & Cattle & Slovakia & AF298068 & [165] \\
\hline BVDV-1.10 (Q) & $11 \mathrm{~N} 36$ & Cattle & China & JX437156 & {$[4]$} \\
\hline BVDV-1.10 (Q) & BJ1022 & Cattle & China & KF925508 & [154] \\
\hline BVDV-1-10 (Q) & BJ1123 & Cattle & China & KF925506 & [154] \\
\hline BVDV-1.10 (Q) & BJ1301 & Cattle & China & KF925503 & [154] \\
\hline BVDV-1.10 (Q) & Camel6 & Bactrian camel & China & KC695810 & [3] \\
\hline BVDV-1.10 (Q) & FJ1003 & Pig & China & JN248728 & [32] \\
\hline BVDV-1.10 (Q) & JL1001 & Pig & China & JN248733 & [32] \\
\hline BVDV-1.10 (M) & isolate 6 & Bactrian camel & China & JX276543 & [31] \\
\hline BVDV-1.10 (M) & isolate 7 & Bactrian camel & China & JX276544 & [31] \\
\hline BVDV-1.10 (Q) & S53 & Cattle & China & KF006962 & {$[8]$} \\
\hline BVDV-1.10 (Q) & S83 & Cattle & China & KF006963 & [8] \\
\hline BVDV-1.10 (Q) & SD0803 & Pig & China & JN400273 & [107] \\
\hline BVDV-1.10 (Q) & ZJ1003 & Pig & China & JN248744 & [32] \\
\hline
\end{tabular}




\section{Continued}

\begin{tabular}{|c|c|c|c|c|c|}
\hline BVDV-1.10 (Q) & ZJ1005 & Pig & China & JN248745 & {$[32]$} \\
\hline BVDV-1.11 (E) & S182 & Cattle & China & KF006965 & {$[8]$} \\
\hline BVDV-1.12.2 & $11207 / 98$ & Cattle & Germany & AJ304390 & {$[154]$} \\
\hline BVDV-1.13 & KS86-1ncp & Cattle & Japan & $\mathrm{AB} 042713$ & [90] \\
\hline BVDV-1.14 (R) & TR70 & Cattle & Turkey & MG670547 & {$[15]$} \\
\hline BVDV-1.14 (R) & TR75 & Cattle & Turkey & MG670549 & {$[15]$} \\
\hline BVDV-1.15.1 & BJ10_10 & Cattle & China & HQ879791 & Zhang et al., unpublish \\
\hline BVDV-1.15.1 (M) & BJ1020 & Cattle & China & KF925511 & {$[6]$} \\
\hline BVDV-1.15.1 & BJ11_01 & Cattle & China & JN542505 & Zhang et al., unpublish \\
\hline BVDV-1.15.1 (M) & BJ1125 & Cattle & China & KF925519 & {$[6]$} \\
\hline BVDV-1.15.1 (M) & BJ1202 & Cattle & China & KF925514 & {$[154]$} \\
\hline BVDV-1.15.1 (M) & BJ1302 & Cattle & China & KF925520 & {$[154]$} \\
\hline BVDV-1.15.1 (M) & BJ1303 & Cattle & China & KF925518 & {$[154]$} \\
\hline BVDV-1.15.1 (M) & BJ1305 & Cattle & China & KF925505 & [154] \\
\hline BVDV-1.15.1 (M) & DG07 & Cattle & China & GU120250 & [7] \\
\hline BVDV-1.15.1 (M) & GXBH-EB20 & Buffalo & China & KJ578811 & [2] \\
\hline BVDV-1.15.1 (M) & GXBH-EB33 & Buffalo & China & KJ578812 & [2] \\
\hline BVDV-1.15.1 (M) & GXBS-LB3 & Buffalo & China & KJ578818 & [2] \\
\hline BVDV-1.15.1 (M) & GXHZ-JB24 & Buffalo & China & KJ578810 & [2] \\
\hline BVDV-1.15.1 (M) & GXLZ-BB13 & Buffalo & China & KJ578817 & [2] \\
\hline BVDV-1.15.1 (M) & GXLZ-BB5 & Buffalo & China & KJ578815 & [2] \\
\hline BVDV-1.15.1 (M) & GXLZ-BB6 & Buffalo & China & KJ578816 & [2] \\
\hline BVDV-1.15.1 (M) & GXYL-KB22 & Buffalo & China & KJ578820 & [2] \\
\hline BVDV-1.15.1 (M) & GXYL-KB53 & Buffalo & China & KJ578821 & [2] \\
\hline BVDV-1.15.1 (M) & HB-0244 & Cattle & China & KJ578837 & [2] \\
\hline BVDV-1.15.1 (M) & HB- 0273 & Cattle & China & KJ578831 & [2] \\
\hline BVDV-1.15.1 (M) & HB-0275 & Cattle & China & KJ578834 & [2] \\
\hline BVDV-1.15.1 (M) & HB-050030 & Cattle & China & KJ578835 & [2] \\
\hline BVDV-1.15.1 (M) & HB-050057 & Cattle & China & KJ578841 & [2] \\
\hline BVDV-1.15.1 (M) & HB-090913 & Cattle & China & KJ578824 & [2] \\
\hline BVDV-1.15.1 (M) & HZ05 & Cattle & China & GU120240 & [7] \\
\hline BVDV-1.15.1 (M) & HZ0601 & Cattle & China & GU120244 & [7] \\
\hline BVDV-1.15.1 (M) & HZ0602 & Cattle & China & GU120245 & [7] \\
\hline BVDV-1.15.1(M) & isolate 11 & Bactrian camel & China & JX276548 & [31] \\
\hline BVDV-1.15.1 (M) & JS-01159 & Cattle & China & KJ578843 & [2] \\
\hline BVDV-1.15.1 (M) & JS-03105 & Cattle & China & KJ578845 & [2] \\
\hline BVDV-1.15.1 (M) & JS-04198 & Cattle & China & KJ578844 & {$[2]$} \\
\hline BVDV-1.15.1 (M) & JS-3094 & Cattle & China & KJ578842 & {$[2]$} \\
\hline BVDV-1.15.1 (M) & LN-1 & Cattle & China & KT896495 & Zhang, unpublish \\
\hline BVDV-1.15.1 (M) & LN317-6 & Cattle & China & KJ578795 & {$[2]$} \\
\hline BVDV-1.15.1 (M) & LZ05 & Cattle & China & GU120241 & [7] \\
\hline BVDV-1.15.1 (M) & NMG311-2 & Cattle & China & KJ578867 & [2] \\
\hline BVDV-1.15.1 (M) & NMG311-3 & Cattle & China & KJ578865 & {$[2]$} \\
\hline BVDV-1.15.1 (M) & NMG312-26 & Cattle & China & KJ578864 & [2] \\
\hline BVDV-1.15.1 (M) & NMG312-32 & Cattle & China & KJ578863 & {$[2]$} \\
\hline BVDV-1.15.1 (M) & NMG313-28 & Cattle & China & KJ578870 & {$[2]$} \\
\hline BVDV-1.15.1 (M) & NMG313-35 & Cattle & China & KJ578869 & {$[2]$} \\
\hline BVDV-1.15.1 (M) & NMG313-55 & Cattle & China & KJ578868 & [2] \\
\hline BVDV-1.15.1 (M) & NMG314-22 & Cattle & China & KJ578858 & {$[2]$} \\
\hline BVDV-1.15.1 (M) & NMG314-51 & Cattle & China & KJ578860 & {$[2]$} \\
\hline BVDV-1.15.1 (M) & NMG314-65 & Cattle & China & KJ578872 & {$[2]$} \\
\hline
\end{tabular}




\section{Continued}

\begin{tabular}{|c|c|c|c|c|c|}
\hline BVDV-1.15.1 (M) & NMG315-1 & Cattle & China & KJ578861 & [2] \\
\hline BVDV-1.15.1 (M) & NX0801 & Cattle & China & GU120252 & [7] \\
\hline BVDV-1.15.1 (M) & NX0802 & Cattle & China & GU120253 & [7] \\
\hline BVDV-1.15.1 (M) & NX0803 & Cattle & China & GU120254 & [7] \\
\hline BVDV-1.15.1 (M) & QHQL-216 & Yak & China & KJ578881 & [2] \\
\hline BVDV-1.15.1 (M) & QHQL-225 & Yak & China & KJ578882 & {$[2]$} \\
\hline BVDV-1.15.1 (M) & QHQL-268 & Yak & China & KJ578885 & [2] \\
\hline BVDV-1.15.1 (M) & S101 & Cattle & China & KJ690686 & {$[8]$} \\
\hline BVDV-1.15.1 (M) & S102 & Cattle & China & KJ690687 & [8] \\
\hline BVDV-1.15.1 (M) & S12 & Cattle & China & KJ690679 & [8] \\
\hline BVDV-1.15.1 (M) & S122 & Cattle & China & KJ690688 & {$[8]$} \\
\hline BVDV-1.15.1 (M) & S123 & Cattle & China & KF006958 & [8] \\
\hline BVDV-1.15.1 (M) & S13 & Cattle & China & KJ690680 & {$[8]$} \\
\hline BVDV-1.15.1 (M) & S21-China & Cattle & China & KF006961 & [8] \\
\hline BVDV-1.15.1 (M) & S22 & Cattle & China & KF006955 & [8] \\
\hline BVDV-1.15.1 (M) & S41 & Cattle & China & KJ690681 & [8] \\
\hline BVDV-1.15.1 (M) & S52 & Cattle & China & KJ690682 & [8] \\
\hline BVDV-1.15.1 (M) & S62 & Cattle & China & KJ690683 & [8] \\
\hline BVDV-1.15.1 (M) & S71 & Cattle & China & KF006956 & [8] \\
\hline BVDV-1.15.1 (M) & S73 & Cattle & China & KJ690684 & [8] \\
\hline BVDV-1.15.1 (M) & S81 & Cattle & China & KJ690685 & [8] \\
\hline BVDV-1.15.1 (M) & SD-15 & Cattle & China & KR866116 & [10] \\
\hline BVDV-1.15.1 (M) & SH1051 & Pig & China & JN248740 & {$[32]$} \\
\hline BVDV-1.15.1 (M) & TJ0801 & Cattle & China & GU120255 & [7] \\
\hline BVDV-1.15.1 (M) & W4-1 & Pig & China & Not deposited & Zhang, unpublish \\
\hline BVDV-1.15.1 (M) & XA08 & Cattle & China & GU120257 & [7] \\
\hline BVDV-1.15.1 (M) & $X Z-141$ & Yak & China & KJ578907 & [2] \\
\hline BVDV-1.15.1 (M) & ZM-95 & Pig & China & AF526381 & [49] \\
\hline BVDV-1.15.1 (M) & ZD05 & Cattle & China & GU120243 & [7] \\
\hline BVDV-1.15.2 (M) & HB-0105 & Cattle & China & KJ578830 & [2] \\
\hline BVDV-1.15.2 (M) & HB-050062 & Cattle & China & KJ578838 & [2] \\
\hline BVDV-1.15.2 (M) & HB-060111 & Cattle & China & KJ578822 & [2] \\
\hline BVDV-1.15.2 (M) & HB-080141 & Cattle & China & KJ578823 & [2] \\
\hline BVDV-1.15.2 (M) & HB-080146 & Cattle & China & KJ578839 & [2] \\
\hline BVDV-1.15.2 (M) & HB- 080147 & Cattle & China & KJ578840 & [2] \\
\hline BVDV-1.15.2 (M) & HB-090268 & Cattle & China & KJ578832 & [2] \\
\hline BVDV-1.15.2(M) & isolate 10 & Bactrian camel & China & JX276547 & [31] \\
\hline BVDV-1.15.2 (M) & JS-03140 & Cattle & China & KJ578846 & [2] \\
\hline BVDV-1.15.2 (M) & LN309-9 & Cattle & China & KJ578804 & [2] \\
\hline BVDV-1.15.2 (M) & LN314-21 & Cattle & China & KJ578802 & [2] \\
\hline BVDV-1.15.2 (M) & NMG313-1 & Cattle & China & KJ578871 & [2] \\
\hline BVDV-1.15.2 (M) & NMG314-60 & Cattle & China & KJ578859 & [2] \\
\hline BVDV-1.15.2 (M) & QHHY-22 & Yak & China & KJ578903 & [2] \\
\hline BVDV-1.15.2 (M) & QHTJ-303887 & Yak & China & KJ578899 & [2] \\
\hline BVDV-1.15.2 (M) & S72 & Cattle & China & KF006957 & [8] \\
\hline BVDV-1.15.2 (M) & $X Z-103$ & Yak & China & KJ578916 & [2] \\
\hline BVDV-1.15.2 (M) & XZ-109 & Yak & China & KJ578917 & [2] \\
\hline BVDV-1.15.2 (M) & $X Z-133$ & Yak & China & KJ578906 & [2] \\
\hline BVDV-1.15.2 (M) & $X Z-176$ & Yak & China & KJ578905 & [2] \\
\hline BVDV-1.15.2 (M) & $\mathrm{XZ}-24$ & Yak & China & KJ578918 & [2] \\
\hline
\end{tabular}




\section{Continued}

\begin{tabular}{|c|c|c|c|c|c|}
\hline BVDV-1.15.2 (M) & $\mathrm{XZ}-25$ & Yak & China & KJ578908 & [2] \\
\hline BVDV-1.15.2 (M) & $X Z-48$ & Yak & China & KJ578909 & [2] \\
\hline BVDV-1.15.2 (M) & $\mathrm{XZ}-69$ & Yak & China & KJ578910 & [2] \\
\hline BVDV-1.15.2 (M) & $\mathrm{XZ}-71$ & Yak & China & KJ578912 & [2] \\
\hline BVDV-1.15.2 (M) & $\mathrm{XZ}-84$ & Yak & China & KJ578913 & [2] \\
\hline BVDV-1.15.2 (M) & $\mathrm{XZ}-86$ & Yak & China & KJ578914 & {$[2]$} \\
\hline BVDV-1.15.2 (M) & $\mathrm{XZ}-92$ & Yak & China & KJ578915 & {$[2]$} \\
\hline BVDV-1.16 (L) & TR16 & Cattle & Turkey & MG670548 & {$[15]$} \\
\hline BVDV-1.16 (L) & TR72 & Cattle & Turkey & MG670546 & {$[15]$} \\
\hline BVDV-1.16 (L) & TR-2007-A-2368MS & Cattle & Turkey & EU716148 & {$[33]$} \\
\hline BVDV-1.16 (L) & TR-2007-Gu-175454-4695 & Cattle & Turkey & EU716150 & [33] \\
\hline BVDV-1.17 (F) & $\mathrm{J}$ & Cattle & Austria & AF298067 & {$[165]$} \\
\hline BVDV-1.18 (P) & BJ0701 & Cattle & China & GU120247 & [7] \\
\hline BVDV-1.18 (P) & BJ0702 & Cattle & China & GU120248 & [7] \\
\hline BVDV-1.18 (P) & BJ0703 & Cattle & China & GU120249 & [7] \\
\hline BVDV-1.18 (M) & isolate 5 & Bactrian camel & China & JX276542 & {$[31]$} \\
\hline BVDV-1.18 (M) & isolate 8 & Bactrian camel & China & JX276545 & {$[31]$} \\
\hline BVDV-1.18 (P) & TJ06 & Cattle & China & GU120246 & [7] \\
\hline BVDV-1.19 (G) & A & Cattle & Austria & AF298064 & [165] \\
\hline BVDV-1.20.1 (X) & CH-01-08 & Cattle & Switzerland & EU180024 & [54] \\
\hline BVDV-1.21.2 (U) & GXBH-EB34 & Buffalo & China & KJ578813 & {$[2]$} \\
\hline BVDV-1.21.2 (U) & GXBS-LB8 & Buffalo & China & KJ578819 & [2] \\
\hline BVDV-1.21.2 (U) & GXCZ-FB7 & Buffalo & China & KJ578806 & [2] \\
\hline BVDV-1.21.2 (U) & GXCZ-FB22 & Buffalo & China & KJ578807 & {$[2]$} \\
\hline BVDV-1.21.2 (U) & GXCZ-FB25 & Buffalo & China & KJ578808 & {$[2]$} \\
\hline BVDV-1.21.2 (U) & GXHZ-JB11 & Buffalo & China & KJ578809 & {$[2]$} \\
\hline BVDV-1.21.2 (U) & GXLZ-BB4 & Buffalo & China & KJ578814 & [2] \\
\hline BVDV-1.21.2 (U) & HB-090166 & Cattle & China & KJ578836 & {$[2]$} \\
\hline BVDV-1.21.2 (U) & JS-00108 & Cattle & China & KJ578848 & [2] \\
\hline BVDV-1.21.2 (U) & JS-0197 & Cattle & China & KJ578853 & [2] \\
\hline BVDV-1.21.2 (U) & JS-02007 & Cattle & China & KJ578854 & [2] \\
\hline BVDV-1.21.2 (U) & JS-03148 & Cattle & China & KJ578850 & [2] \\
\hline BVDV-1.21.2 (U) & JS-03198 & Cattle & China & KJ578851 & {$[2]$} \\
\hline BVDV-1.21.2 (U) & JS-04119 & Cattle & China & KJ578855 & [2] \\
\hline BVDV-1.21.2 (U) & JS-04138 & Cattle & China & KJ578852 & [2] \\
\hline BVDV-1.21.2 (U) & JS-05002 & Cattle & China & KJ578857 & {$[2]$} \\
\hline BVDV-1.21.2 (U) & JS-99054 & Cattle & China & KJ578847 & {$[2]$} \\
\hline BVDV-1.21.2 (U) & JS-X02126 & Cattle & China & KJ578856 & [2] \\
\hline BVDV-1.21.2 (U) & LN309-5 & Cattle & China & KJ578803 & {$[2]$} \\
\hline BVDV-1.21.2 (U) & M31182 & Yak & China & JQ799141 & Sun et al., unpublish \\
\hline BVDV-1.21.2 (U) & NMG311-20 & Cattle & China & KJ578866 & {$[2]$} \\
\hline BVDV-1.21.2 (U) & NMG315-5 & Cattle & China & KJ578862 & [2] \\
\hline BVDV-1.21.2 (U) & QHQL-252 & Yak & China & KJ578884 & {$[2]$} \\
\hline BVDV-1.22 (K) & Rebe & Cattle & Switzerland & AF299317 & {$[152]$} \\
\hline BVDV-1.23 (P) & S153 & Cattle & China & KF006964 & {$[165]$} \\
\hline BVDV-2a1.1 & 167237 & Sheep & UK & U65055 & {$[164]$} \\
\hline BVDV-2a1.1 & 168149 & Sheep & UK & U65056 & {$[164]$} \\
\hline BVDV-2a1.1 & 173157 & Sheep & UK & U65058 & {$[164]$} \\
\hline BVDV-2a1.1 & 175375 & Sheep & UK & U65059 & {$[164]$} \\
\hline BVDV-2a1.1 & BSE921 & Cattle & Belgium & ALIGN_000012 & {$[104]$} \\
\hline BVDV-2a1.1 & CPA & Contaminant & Japan & D50812 & [30] \\
\hline
\end{tabular}




\section{Continued}

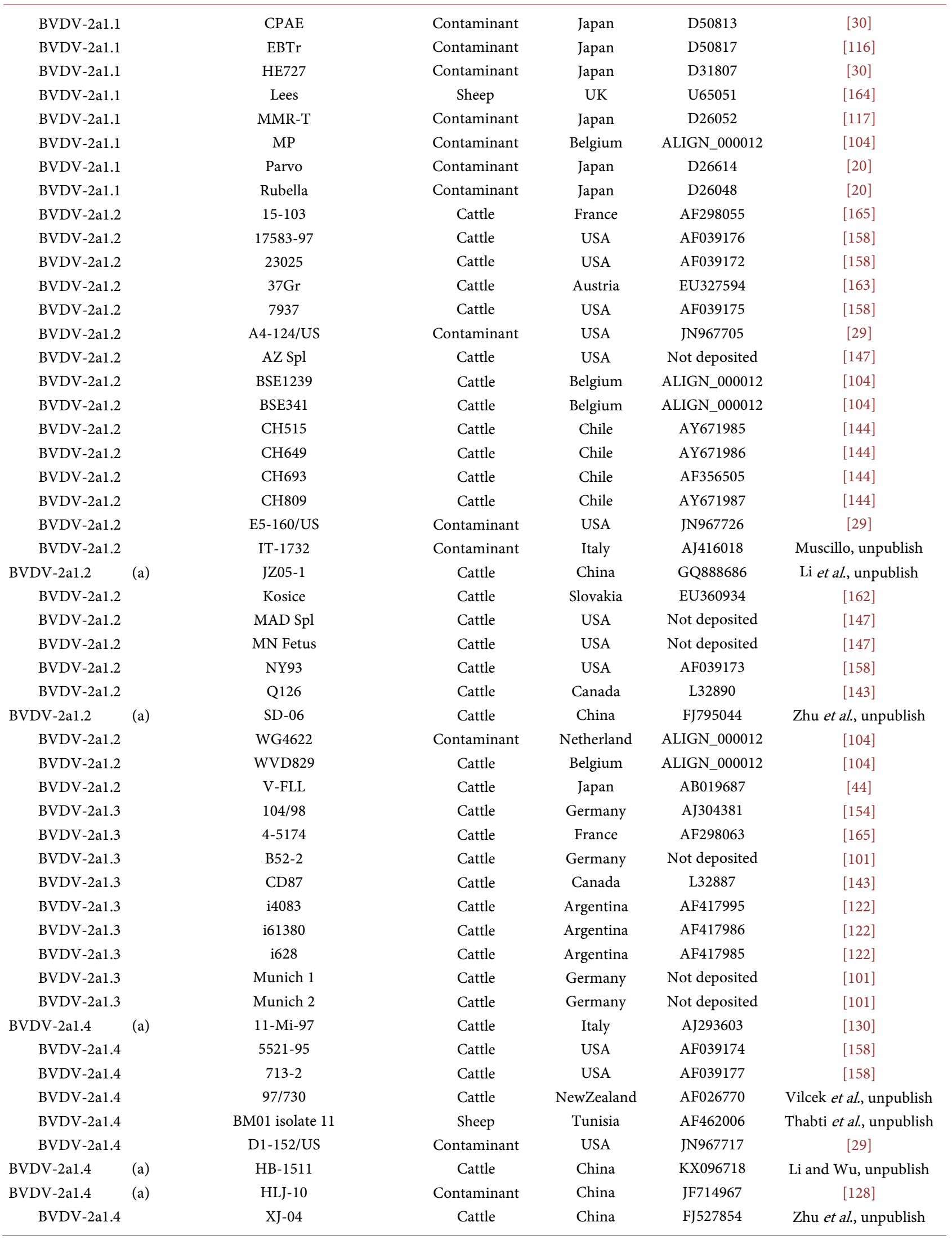


M. Giangaspero et al.

\section{Continued}

\begin{tabular}{|c|c|c|c|c|c|}
\hline BVDV-2a1.4 & SH-28 & Pig & China & HQ258810 & [155] \\
\hline BVDV-2a1.4 & UVR420 & Cattle & Belgium & ALIGN_000012 & [104] \\
\hline BVDV-2a1.5 & BD-78 & Sheep & USA & U18330 & [153] \\
\hline BVDV-2a1.5 & $\mathrm{C} 413$ & Sheep & USA & AF002227 & Chen \& Berry, unpublish \\
\hline BVDV-2a1.5 & $\mathrm{K} 1-2 / \mathrm{CA}$ & Contaminant & Canada & JN967735 & [29] \\
\hline BVDV-2a1.6 & 890 & Cattle & Canada & L32886 & [143] \\
\hline BVDV-2a2.1.1 & AF112 & Cattle & Germany & Not deposited & [101] \\
\hline BVDV-2a2.1.1 & B45-5 & Cattle & Germany & Not deposited & [101] \\
\hline BVDV-2a2.1.1 & B50-5 & Cattle & Germany & Not deposited & [101] \\
\hline BVDV-2a2.1.1 & B5-4 & Cattle & Germany & Not deposited & [101] \\
\hline BVDV-2a2.1.1 & $\mathrm{BA} / 35 / 02$ & Cattle & Italy & AM749820 & [110] \\
\hline BVDV-2a2.1.1 & BS-95-II & Cattle & Italy & AJ288903 & [130] \\
\hline BVDV-2a2.1.1 & E1-198/CA & Contaminant & Canada & JN967719 & [29] \\
\hline BVDV-2a2.1.1 & Giessen-1 & Cattle & Germany & AF104030 & {$[61]$} \\
\hline BVDV-2a2.1.1 & $\mathrm{LO} / 04 / 04$ & Cattle & Italy & AM749821 & [110] \\
\hline BVDV-2a2.1.1 & $\mathrm{LO} / 08 / 04$ & Cattle & Italy & AM749822 & [110] \\
\hline BVDV-2a2.1.1 & $\mathrm{LO} / 10 / 04$ & Cattle & Italy & AM749823 & [110] \\
\hline BVDV-2a2.1.1 & $\mathrm{LO} / 13 / 04$ & Cattle & Italy & AM749824 & [110] \\
\hline BVDV-2a2.1.1 & MS-1 & Cattle & Japan & AB019688 & {$[44]$} \\
\hline BVDV-2a2.1.1 & Munich 3 & Cattle & Germany & Not deposited & [9] \\
\hline BVDV-2a2.1.1 & OY89 & Cattle & Japan & AB003621 & [139] \\
\hline BVDV-2a2.1.1 (a) & PT4-02 & Cattle & Portugal & AY944291 & [99] \\
\hline BVDV-2a2.1.1 & SW90 & Cattle & Japan & AB003622 & [90] \\
\hline BVDV-2a2.1.1 & SY-89 & Cattle & Japan & АВ019689 & [44] \\
\hline BVDV-2a2.1.1 & TC Shinozaki & Cattle & Japan & AB04267 & [90] \\
\hline BVDV-2a2.1.1 & Bov/Ita/187.15-38 & Cattle & Italy & KX350078 & [105] \\
\hline BVDV-2a2.1.1 & Bov/Ita/232.03 & Cattle & Italy & KX350067 & [105] \\
\hline BVDV-2a2.1.1 & $\mathrm{Cp} / \mathrm{Ita} / 124.15-20$ & Goat & Italy & KX350077 & [105] \\
\hline BVDV-2a2.1.1 & $\mathrm{Cp} / \mathrm{Ita} / 124.15-23$ & Goat & Italy & KX350076 & [105] \\
\hline BVDV-2a2.1.1 & Cp/Ita/190.15-36 & Goat & Italy & KX350069 & [105] \\
\hline BVDV-2a2.1.1 & $\mathrm{Cp} / \mathrm{Ita} / 191.15-21$ & Goat & Italy & KX350075 & [105] \\
\hline BVDV-2a2.1.1 & Ov/Ita/182.15-33 & Sheep & Italy & KX350068 & [105] \\
\hline BVDV-2a2.1.1 & Ov/Ita/188.15-18 & Sheep & Italy & KX350074 & [105] \\
\hline BVDV-2a2.1.1 & Ov/Ita/189.15-97 & Sheep & Italy & KX350072 & [105] \\
\hline BVDV-2a2.1.1 & Ov/Ita/189.15-69 & Sheep & Italy & KX350073 & [105] \\
\hline BVDV-2a2.1.1 & Ov/Ita/191.15-16 & Sheep & Italy & KX350071 & [105] \\
\hline BVDV-2a2.1.1 & Ov/Ita/192.15 & Sheep & Italy & KX350070 & [105] \\
\hline BVDV-2a2.1.2 & $17011-96$ & Cattle & USA & AF039179 & [158] \\
\hline BVDV-2a2.2 & B77-5 & Cattle & Germany & Not deposited & [101] \\
\hline BVDV-2a3 (c) & $301 / 09$ & Cattle & Italy & KY040413 & [88] \\
\hline BVDV-2b1.1 & $\mathrm{F} 1-5 / \mathrm{BR}$ & Contaminant & Brazil & JN967743 & [29] \\
\hline BVDV-2b1.1 & VS-63 & Cattle & Brazil & AF410789 & [109] \\
\hline BVDV-2b1.2 & VS-123.4 & Cattle & Brazil & AF410790 & [109] \\
\hline BVDV-2b1.3 & $34 \mathrm{~b}$ & Cattle & Argentina & AF244952 & [121] \\
\hline BVDV-2b1.3 & ncp7 & Cattle & Argentina & Not deposited & {$[121]$} \\
\hline BVDV-2b1.4 & $4 \mathrm{p}$ & Human & Brazil & MG436781 & Tanuri et al., unpublish \\
\hline BVDV-2b1.4 & LV56-10/13 & Cattle & Brazil & KM007124 & [69] \\
\hline BVDV-2b1.4 & LV/Patol02/09 & Cattle & Brazil & KP715137 & Silveira et al., unpublish \\
\hline BVDV-2b1.5 & Giessen 6 & Cattle & Germany & AY379547 & Kuehne et al., unpublish \\
\hline BVDV-2b1.5 & VM96 & Cattle & Brazil & KP715141 & Silveira et al., unpublish \\
\hline BVDV-2b1.5 & VS-260 & Cattle & Brazil & AF410788 & [109] \\
\hline
\end{tabular}




\section{Continued}

\begin{tabular}{|c|c|c|c|c|c|}
\hline BVDV-2b1.5 & LV-96 & Cattle & Brazil & AF410787 & [109] \\
\hline BVDV-2b2 & B5-135/MX & Contaminant & Mexico & JN967713 & [29] \\
\hline BVDV-2b3 & Soldan & Cattle & Brazil & U94914 & Canal et al., unpublish \\
\hline BVDV-2b4 (b) & SD-1301 & Cattle & China & KJ000672 & [5] \\
\hline BVDV-2b4 (b) & S143 & Contaminant & China & KF006972 & [8] \\
\hline BVDV-2b4 (b) & S172 & Contaminant & China & KF006973 & [8] \\
\hline BVDV-2b4 (b) & S51 & Contaminant & China & Not deposited & {$[8]$} \\
\hline BVDV-2c & 098 & Sheep & Tunisia & AF462004 & Thabti et al., unpublish \\
\hline BVDV-2c & 119 & Sheep & Tunisia & AF462003 & Thabti et al., unpublish \\
\hline BVDV-2c & 63 & Sheep & Tunisia & AF462005 & Thabti et al., unpublish \\
\hline BVDV-2d & A3-118/US & Contaminant & USA & JN967703 & {$[29]$} \\
\hline BVDV-2e & i33283 & Cattle & Argentina & AF417996 & {$[122]$} \\
\hline BVDV-2f1 & 354 & Contaminant & Argentina & AF244959 & {$[121]$} \\
\hline BVDV-2f2 (b) & PT2-01 & Cattle & Portugal & AY944277 & [99] \\
\hline BVDV-2f2 (b) & PT7-02 & Cattle & Portugal & AY944297 & [99] \\
\hline BVDV-2f2 & Ptn3 & Cattle & Portugal & EU034173 & Mota, unpublish \\
\hline BVDV-2f 2 & Ptn4 & Cattle & Portugal & EU034174 & Mota, unpublish \\
\hline BVDV-2f2 & Ptn8 & Cattle & Portugal & EU034175 & Mota, unpublish \\
\hline BVDV-2nd & 59386 & Sheep & UK & U17146 & {$[100]$} \\
\hline BVDV-2nd & SCP & Contaminant & UK & U17148 & [100] \\
\hline BVDV-3.1.1.1 & $\mathrm{A} 2 / \mathrm{BR}$ & Contaminant & Brazil & JN967701 & [29] \\
\hline BVDV-3.1.1.1 & $\mathrm{Au} / \mathrm{A} 55110-1162 / 09$ & Contaminant & Australia & FR873797 & [167] \\
\hline BVDV-3.1.1.1 & $\mathrm{Au} / 9 \mathrm{SB} 007 / 09$ & Contaminant & Australia & FR873798 & [167] \\
\hline BVDV-3.1.1.1 & $\mathrm{B} 1 / \mathrm{AU}$ & Contaminant & Australia & JN967707 & [29] \\
\hline BVDV-3.1.1.1 & $\mathrm{B} 2-42 / \mathrm{AU}$ & Contaminant & Australia & JN967709 & [29] \\
\hline BVDV-3.1.1.1 & $\mathrm{B} 3 / \mathrm{AU}$ & Contaminant & Australia & JN967710 & {$[29]$} \\
\hline BVDV-3.1.1.1 & $\mathrm{B} 4-3 / \mathrm{CA}$ & Contaminant & Canada & JN967711 & [29] \\
\hline BVDV-3.1.1.1 & $\mathrm{B} 5-3 / \mathrm{MX}$ & Contaminant & Mexico & JN967747 & [29] \\
\hline BVDV-3.1.1.1 & B6/US & Contaminant & USA & JN967714 & [29] \\
\hline BVDV-3.1.1.1 & D32/00_HoBi/Brazil/200/2002 & Cattle & Brazil & EF683557 & [98] \\
\hline BVDV-3.1.1.1 & D32/00_HoBi & Cattle & Brazil & AY489116 & [84] \\
\hline BVDV-3.1.1.1 & $\mathrm{E} 4 / \mathrm{SA}$ & Contaminant & South American & JN967724 & [29] \\
\hline BVDV-3.1.1.1 & F1-29/BR & Contaminant & Brazil & JN967727 & [29] \\
\hline BVDV-3.1.1.1 & G1-35/AU & Contaminant & Australia & JN967728 & [29] \\
\hline BVDV-3.1.1.1 & $\mathrm{G} 2 / \mathrm{BR}$ & Contaminant & Brazil & JN967729 & [29] \\
\hline BVDV-3.1.1.1 & $\mathrm{H} 1-50 / \mathrm{AU}$ & Contaminant & Australia & JN967731 & [29] \\
\hline BVDV-3.1.1.1 & $\mathrm{H} 3-3 / \mathrm{US}$ & Contaminant & USA & JN967748 & [29] \\
\hline BVDV-3.1.1.1 & Hobi/Brazil/315/2004 & Cattle & Brazil & EF683558 & [98] \\
\hline BVDV-3.1.1.1 & Italy-1/10-1 & Cattle & Italy & HQ231763 & [85] \\
\hline BVDV-3.1.1.1 & Italy-280/11-A & Cattle & Italy & JN703311 & [86] \\
\hline BVDV-3.1.1.1 & Italy-83/10-cp & Cattle & Italy & JQ612705 & [87] \\
\hline BVDV-3.1.1.1 & Italy-83/10-ncp & Cattle & Italy & JQ612704 & [87] \\
\hline BVDV-3.1.1.1 & IZSPLV_To & Contaminant & Italy & HM151361 & [142] \\
\hline BVDV-3.1.1.1 & LV02/12 & Cattle & Brazil & KC465389 & Weber et al., unpublish \\
\hline BVDV-3.1.1.1 & LV03/12 & Cattle & Brazil & KC465390 & Weber et al., unpublish \\
\hline BVDV-3.1.1.1 & LV04/12 & Cattle & Brazil & KC465391 & Weber et al., unpublish \\
\hline BVDV-3.1.1.1 & LPV-WR/BR11 & Cattle & Brazil & KC544256 & Cruz et al., unpublish \\
\hline BVDV-3.1.1.1 & PB22487 & Cattle & Brazil & KY762287 & {$[136]$} \\
\hline BVDV-3.1.1.1 & SV478/07 & Cattle & Brazil & KY767958 & {$[136]$} \\
\hline BVDV-3.1.1.2 & $\mathrm{JS} 12 / 01$ & Contaminant & China & JX469119 & [35] \\
\hline BVDV-3.1.1.3 & Th/04_KhonKaen & Cattle & Thailand & DQ897641 & [36] \\
\hline
\end{tabular}




\section{Continued}

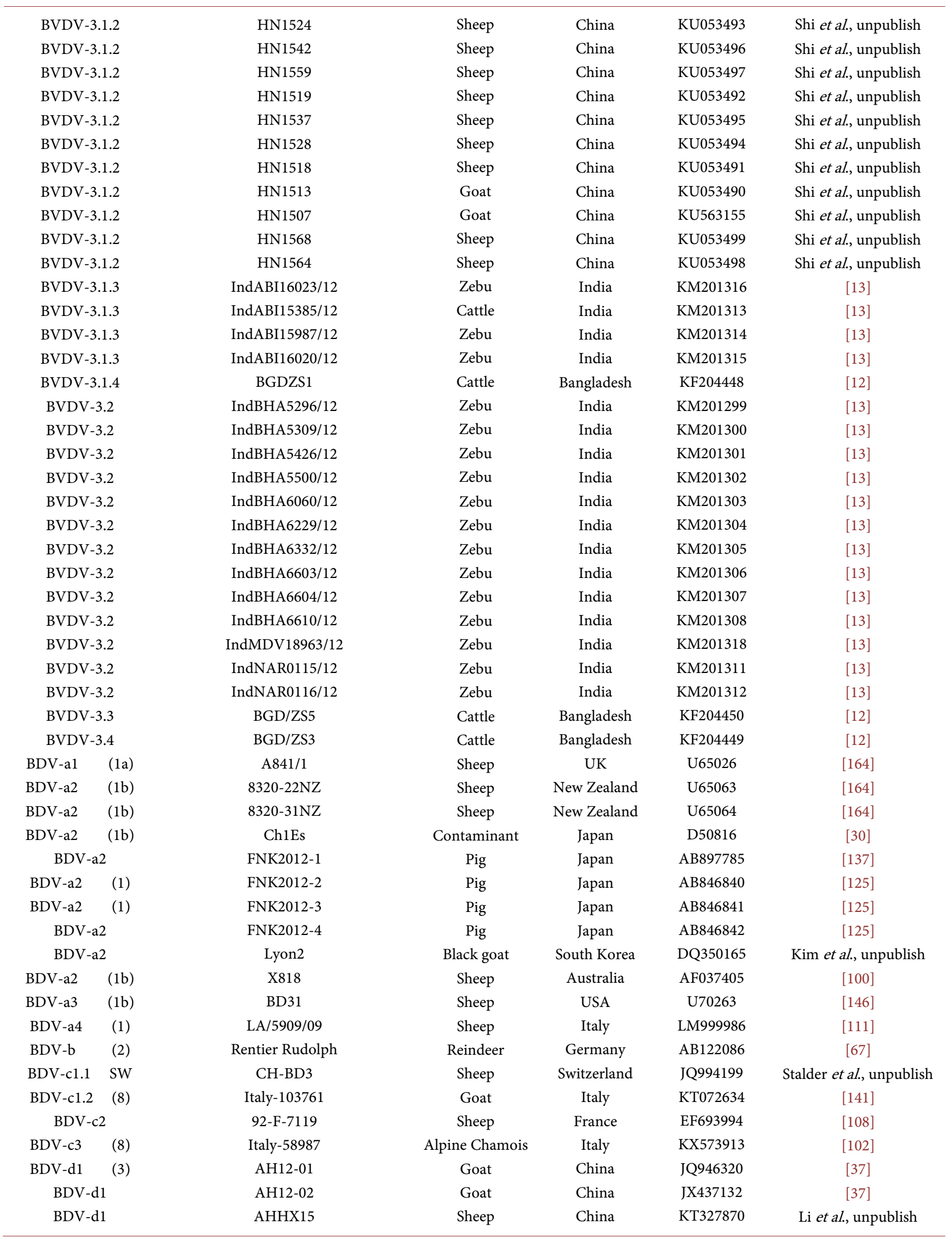




\section{Continued}

\begin{tabular}{|c|c|c|c|c|c|c|}
\hline BDV-d2 & (3) & 297 & Sheep & Slovakia & KC484999 & [38] \\
\hline BDV-e1 & $(4)$ & $\mathrm{C} 27$ & Sheep & Spain & DQ275623 & {$[161]$} \\
\hline BDV-e2 & $(4 b)$ & ZA1-1115 & Sheep & Spain & DQ361070 & [160] \\
\hline BDV-f1 & (5) & $\mathrm{AV}$ & Sheep & France & EF693984 & [108] \\
\hline BDV-f2 & $(4 c)$ & $2112 / 99$ & Sheep & Spain & AY159513 & {$[70]$} \\
\hline $\mathrm{BDV}-\mathrm{f} 3$ & $(4 b)$ & Rocco & Sheep & Spain & DQ361067 & {$[160]$} \\
\hline BDV-f4 & (4) & Chamois-1 (H2121) & Pyrenean chamois & Andorra & AY738080 & {$[64]$} \\
\hline \multicolumn{2}{|c|}{ BDV-g1 } & 91-F-6732 & Sheep & France & EF988633 & [108] \\
\hline BDV-g2 & $\mathrm{TU}$ & $37 \mathrm{~A}$ & Contaminant & Tunisia & AF461999 & [156] \\
\hline BDV-h & (3) & JS12/04 & Goat & China & KC537789 & [37] \\
\hline \multicolumn{2}{|c|}{ BDV-h } & JSLS12-01 & Sheep & China & КС963426 & [129] \\
\hline \multicolumn{2}{|c|}{ BDV-h } & JSYZ15 & Sheep & China & KT327869 & Li et al., unpublish \\
\hline \multicolumn{2}{|c|}{ BDV-i1 } & TR-13 & Sheep & Turkey & JF489888 & [39] \\
\hline \multicolumn{2}{|c|}{ BDV-i2 } & TR-14 & Sheep & Turkey & JF489889 & [39] \\
\hline$B D V-j 1$ & (3) & Ind 830-09 & Sheep & India & KT934377 & {$[40]$} \\
\hline$B D V-j 2$ & (3) & $\mathrm{CH}-\mathrm{BD} 1$ & Sheep & Switzerland & JQ994201 & Stalder, et al., unpublish \\
\hline$B D V-j 3$ & (3) & Gifhorn & Pig & Germany & EU636997 & Schirrmeier et al., unpublish \\
\hline \multicolumn{2}{|c|}{ Pestivirus I } & BDV/Aydin/04-TR & Sheep & Turkey & AM418427 & {$[57]$} \\
\hline \multicolumn{2}{|c|}{ Pestivirus I } & BDV/Burdur/05-TR & Sheep & Turkey & AM418428 & {$[57]$} \\
\hline \multicolumn{2}{|c|}{ CSFV-a1 } & 1 India & Cattle & India & MG859286 & [11] \\
\hline \multicolumn{2}{|c|}{ CSFV-a1 } & 10 India & Cattle & India & MK105823 & [11] \\
\hline \multicolumn{2}{|c|}{ CSFV-a1 } & 11 India & Cattle & India & MG859287 & [11] \\
\hline \multicolumn{2}{|c|}{ CSFV-a1 } & 13 India & Cattle & India & MK105824 & {$[11]$} \\
\hline \multicolumn{2}{|c|}{ CSFV-a1 } & 2 India & Cattle & India & MK105825 & [11] \\
\hline \multicolumn{2}{|c|}{ CSFV-a1 } & 39 & Pig & China & AF407339 & Wu et al., unpublish \\
\hline \multicolumn{2}{|c|}{ CSFV-a1 } & 3 India & Cattle & India & MK105826 & [11] \\
\hline \multicolumn{2}{|c|}{ CSFV-a1 } & 4 India & Cattle & India & MK105820 & [11] \\
\hline \multicolumn{2}{|c|}{ CSFV-al } & 5 India & Cattle & India & MK105821 & [11] \\
\hline \multicolumn{2}{|c|}{ CSFV-a1 } & 5NCR/CSF/MZ/AIZ/348 & Pig & India & JX975460 & Rajkhowa et al., unpublish \\
\hline \multicolumn{2}{|c|}{ CSFV-a1 } & 5NCR/CSF/MZ/AIZ/352 & Pig & India & JX975461 & Rajkhowa et al., unpublish \\
\hline \multicolumn{2}{|c|}{ CSFV-a1 } & 6 India & Cattle & India & MK105822 & {$[11]$} \\
\hline \multicolumn{2}{|c|}{ CSFV-a1 } & 7 India & Cattle & India & MK109913 & [11] \\
\hline CSFV & & 8 India & Cattle & India & MG813566 & [11] \\
\hline CSFV & & 9 India & Cattle & India & MK105827 & [11] \\
\hline CSFV-a1 & $(1.1)$ & Alfort 187 & Pig & France & X87939 & [149] \\
\hline CSFV & & Alfort A19 & Pig & France & U90951 & Smondack et al., unpublish \\
\hline CSFV & & Aizawl-09 & Pig & India & HM449066 & Rajkhowa et al., unpublish \\
\hline CSFV & & Bangalore Ind-163/07 & Pig & India & EU446419 & Patil et al., unpublish \\
\hline CSFV-a1 & $(1.1)$ & Brescia & Pig & Italy & M31768 & [134] \\
\hline CSFV-a1 & $(1.2)$ & BRESCIAX & Pig & Italy & AY578687 & {$[148]$} \\
\hline CSFV & & BV-P & Pig & Hungary & DQ314582 & Farsang et al., unpublish \\
\hline CSFV & & C strain & Vaccine & China & Z46258 & [135] \\
\hline CSFV-al & $(1.1)$ & CAP & Pig & Switzerland & X96550 & Tratschin et al., unpublish \\
\hline CSFV & & $\mathrm{cF} 114$ & Pig & China & AF333000 & Mingxiao et al., unpublish \\
\hline CSFV & & $\mathrm{CSF} / \mathrm{MZ} / \mathrm{KOL} / 73$ & Pig & India & JX094153 & Rajkhowa, unpublish \\
\hline CSFV & & CSF/MZ/SAI/76 & Pig & India & JX094154 & Rajkhowa, unpublish \\
\hline CSFV-a1 & $(1.1)$ & CSFV/1.1/dp/CSF0382/Koslov & Pig & Czech Republic & HM237795 & [126] \\
\hline CSFV-a1 & $(2.3)$ & CSFV/2.3/wb/0608/2005/Euskirchen & Wild boar & Germany & GU233732 & [126] \\
\hline CSFV-a1 & $(2.3)$ & CSFV/2.3/wb/CSF1046/2009/Hennef & Wild boar & Germany & GU233733 & [126] \\
\hline CSFV-a1 & $(2.3)$ & CSFV/2.3/wb/CSF1045/2009/Roesrath & Pig & Germany & LT593749 & Hoeper, unpublish \\
\hline CSFV-a1 & $(1.1)$ & CSFV212L-13 & Pig & India & KY860615 & Tomar et al., unpublish \\
\hline
\end{tabular}




\section{Continued}

\begin{tabular}{|c|c|c|c|c|c|c|}
\hline CSFV-a1 & $(1.1)$ & CSFV-GZ-2009 & Pig & China & HQ380231 & [150] \\
\hline CSFV-a1 & $(1.1)$ & CSFV/IVRI/VB-131 & Pig & India & KM262189 & [124] \\
\hline CSFV-a1 & $(1.1)$ & CSFV-PK15C-NG79-11 & Contaminant & India & KC503764 & [157] \\
\hline \multicolumn{2}{|c|}{ CSFV-a1 } & Eystrup & Pig & Germany & AF326963 & [133] \\
\hline \multicolumn{2}{|c|}{ CSFV-a1 } & GPE (-) & Vaccine & Japan & AB019152 & {$[43]$} \\
\hline CSFV-a1 & $(1.1)$ & HCLV & Vaccine & India & AF091507 & Wang et al, unpublish \\
\hline CSFV-a1 & (1.1) & HCVCAD22/14 & Contaminant & India & U606028 & Desai et al., unpublish \\
\hline \multicolumn{2}{|c|}{ CSFV-a1 } & Hokkaido/66 & Pig & Japan & AB019154 & {$[43]$} \\
\hline \multicolumn{2}{|c|}{ CSFV-a1 } & Ibaraki/66 & Pig & Japan & AB019156 & [43] \\
\hline \multicolumn{2}{|c|}{ CSFV-a1 } & Ibaraki/81-115 & Pig & Japan & AB019158 & {$[43]$} \\
\hline \multicolumn{2}{|c|}{ CSFV-a1 } & Ibaraki/81-20 & Pig & Japan & AB019160 & {$[43]$} \\
\hline \multicolumn{2}{|c|}{ CSFV-a1 } & Ibaraki/81-38 & Pig & Japan & AB019162 & {$[43]$} \\
\hline \multicolumn{2}{|c|}{ CSFV-a1 } & Ibaraki/81-40 & Pig & Japan & AB019164 & {$[43]$} \\
\hline \multicolumn{2}{|c|}{ CSFV-a1 } & Ind-173/08 & Pig & India & FJ183444 & [140] \\
\hline \multicolumn{2}{|c|}{ CSFV-a1 } & Ind-174/08 & Pig & India & FJ183445 & [140] \\
\hline \multicolumn{2}{|c|}{ CSFV-a1 } & Ind-175/08 & Pig & India & FJ183446 & [140] \\
\hline \multicolumn{2}{|c|}{ CSFV-a1 } & Ind-176/08 & Pig & India & FJ183447 & [140] \\
\hline \multicolumn{2}{|c|}{ CSFV-a1 } & Ind-239/08 & Pig & India & FJ183449 & [140] \\
\hline \multicolumn{2}{|c|}{ CSFV-a1 } & Ind-243/08 & Pig & India & FJ183452 & [140] \\
\hline \multicolumn{2}{|c|}{ CSFV-a1 } & Ind-272/08 & Pig & India & FJ183456 & [140] \\
\hline CSFV-a1 & $(1.1)$ & $J L 1(06)$ & Pig & China & EU497410 & Qiu et al., unpublish \\
\hline \multicolumn{2}{|c|}{ CSFV-a1 } & KC & Vaccine & Russia & AF099102 & [114] \\
\hline \multicolumn{2}{|c|}{ CSFV-a1 } & LK-VNIVViM & Vaccine & Russia & KM522833 & [169] \\
\hline CSFV-a1 & (1.1) & LOM & Pig & Japan & AB019655 & {$[44]$} \\
\hline \multicolumn{2}{|c|}{ CSFV-a1 } & Miyazaki/81 & Pig & Japan & AB019168 & {$[43]$} \\
\hline \multicolumn{2}{|c|}{ CSFV-a1 } & Nakamura/66 & Pig & Japan & AB019170 & {$[43]$} \\
\hline CSFV-a1 & (1.1) & NFP/AS-1 & Pig & India & KC617749 & Roychoudhury et al, unpublish \\
\hline CSFV-a1 & (1.1) & NFP/ML-2 & Pig & India & KC617761 & Roychoudhury et al, unpublish \\
\hline CSFV-a1 & (1.1) & NFP/ML-4 & Pig & India & KC617750 & Roychoudhury et al, unpublish \\
\hline \multicolumn{2}{|c|}{ CSFV-a1 } & Rovac & Vaccine & USA & KJ873238 & [169] \\
\hline CSFV-a1 & $(1.2)$ & RUCSFPLUM & Vaccine & USA & AY578688 & {$[148]$} \\
\hline CSFV & & Shimen & Pig & China & AF092448 & Huang et al., unpublish \\
\hline CSFV & & SWH & Pig & China & DQ127910 & [127] \\
\hline CSFV & & Vac A & Pig & USA & L42435 & [151] \\
\hline CSFV & & Yamanashi/69 & Pig & Japan & AB019182 & [43] \\
\hline CSFV & & $17-93$ & Pig & Poland & L42413 & [151] \\
\hline CSFV-a2 & $(2.3)$ & Alfort/Tübingen & Pig & France & J04358 & [132] \\
\hline CSFV & & Chiba-80 & Pig & Japan & AB019659 & {$[44]$} \\
\hline CSFV-a2 & $(2.3)$ & CSFV/2.3/dp/CSF0821/HR/Novska & Pig & Croatia & HQ148061 & Leifer et al., unpublish \\
\hline CSFV-a2 & $(2.3)$ & CSFV/2.3/dp/CSF857/Borken & Pig & Germany & GU233731 & {$[126]$} \\
\hline CSFV-a2 & $(2.3)$ & $\mathrm{CSFV} / 2.3 / \mathrm{dp} / \mathrm{CSF} 864 / \mathrm{BG} / \mathrm{Jambul}$ & Pig & Bulgaria & HQ148062 & Leifer et al., unpublish \\
\hline CSFV-a2 & $(2.3)$ & CSFV/2.3/wb/XXX0609/Uelzen & Wild boar & Germany & GU324242 & [126] \\
\hline CSFV-a2 & $(2.3)$ & $\mathrm{CSFV} / 2.3 / \mathrm{SRB} / 1264 / 2005$ & Pig & Serbia & KY849593 & Petrovic et al., unpublish \\
\hline CSFV-a2 & $(2.3)$ & $\mathrm{CSFV} / 2.3 / \mathrm{SRB} / 6168 / 2006$ & Pig & Serbia & KY849594 & Petrovic et al., unpublish \\
\hline CSFV & & CSF/wb/FR57/2004/0964-03 & Pig & Germany & LT158404 & Hoeper, unpublish \\
\hline CSFV & & CSF/wb/FR67/2003/0647-19 & Pig & Germany & LT158502 & Hoeper, unpublish \\
\hline CSFV & & CSF/wb/FR67/2005/0018-06 & Pig & Germany & LT158405 & Hoeper, unpublish \\
\hline CSFV & & CSF/wb/FR67/2005/0125-05 & Pig & Germany & LT158406 & Hoeper, unpublish \\
\hline CSFV & & CSF/wb/FR67/2005/0238-02 & Pig & Germany & LT158407 & Hoeper, unpublish \\
\hline CSFV & & CSF/wb/FR67/2006/0199-01 & Pig & Germany & LT158409 & Hoeper, unpublish \\
\hline CSFV & & CSF/wb/FR67/2006/060003-06 & Pig & Germany & LT158408 & Hoeper, unpublish \\
\hline
\end{tabular}


Continued

\begin{tabular}{|c|c|c|c|c|c|c|}
\hline \multicolumn{2}{|c|}{ CSFV-a2 } & CSF/wb/FR67/2007/0192-01 & Pig & Germany & LT158410 & Hoeper, unpublish \\
\hline CSFV-a2 & $(2.1 \mathrm{~g})$ & GD19/2011 & Pig & China & KU504339 & [113] \\
\hline \multicolumn{2}{|c|}{ CSFV-a2 } & HEN03 & Cattle & China & KC176778 & Zhang et al., unpublish \\
\hline CSFV-a2 & $(2.1)$ & HNLY-2011 & Pig & China & JX262391 & [120] \\
\hline CSFV-a2 & $(2.1)$ & HNSD-2012 & Pig & China & JX218094 & [120] \\
\hline \multicolumn{2}{|c|}{ CSFV-a2 } & Osaka/51 & Pig & Japan & AB019174 & [43] \\
\hline \multicolumn{2}{|c|}{ CSFV-a2 } & Osaka/71 & Pig & Japan & AB019176 & [43] \\
\hline \multicolumn{2}{|c|}{ CSFV-a2 } & Pader & Pig & Garmany & AY072924 & [159] \\
\hline \multicolumn{2}{|c|}{ CSFV-a2 } & Paderborn & Pig & Garmany & GQ902941 & [145] \\
\hline \multicolumn{2}{|c|}{ CSFV-a2 } & S112 & Contaminant & China & MK118725 & {$[8]$} \\
\hline \multicolumn{2}{|c|}{ CSFV-a2 } & S173 & Contaminant & China & KF006975 & {$[8]$} \\
\hline \multicolumn{2}{|c|}{ CSFV-a2 } & Shizuoka/73 & Pig & Japan & AB019180 & [43] \\
\hline CSFV-a2 & $(2.1)$ & SKCDK & Pig & China & GQ923951 & Li et al., unpublish \\
\hline \multicolumn{2}{|c|}{ CSFV-a2 } & Sp01 & Pig & Spain & FJ265020 & Mena et al, unpublish \\
\hline \multicolumn{2}{|c|}{ CSFV-a2 } & Switzerland 1/93 & Pig & Switzerland & AF045068 & [119] \\
\hline \multicolumn{2}{|c|}{ CSFV-a2 } & Switzerland 2/93' & Pig & Switzerland & AF045069 & [119] \\
\hline \multicolumn{2}{|c|}{ CSFV-a2 } & Switzerland 3/93/1' & Pig & Switzerland & AF045070 & [119] \\
\hline \multicolumn{2}{|c|}{ CSFV-a2 } & Switzerland 3/93/2' & Pig & Switzerland & AF045071 & [119] \\
\hline \multicolumn{2}{|c|}{ CSFV-a2 } & Switzerland 4/93' & Pig & Switzerland & AF045072 & [119] \\
\hline \multicolumn{2}{|c|}{ CSFV-a2 } & Venhorst & Pig & Netherlands & AF084049 & [166] \\
\hline \multicolumn{2}{|c|}{ CSFV-a2 } & VRI4762 & Pig & Malaysia & L42437 & [151] \\
\hline CSFV-a2 & $(2.3)$ & Wingene & Pig & Belgium & JQ595295 & Haegeman et al., unpublish \\
\hline \multicolumn{2}{|c|}{ CSFV-a3 } & Saitama/81 & Pig & Japan & AB019178 & [43] \\
\hline CSFV-a4 & $(2.2)$ & 179/MIB/2014 & Pig & India & KR350485 & [42] \\
\hline CSFV-a4 & $(2.2)$ & 181/MIB/2014 & Pig & India & KR350486 & {$[42]$} \\
\hline CSFV-a4 & $(2.2)$ & 211/MIB/2014 & Wild boar & India & KR350487 & Ravishankar et al., unpublish \\
\hline CSFV-a4 & $(2.2)$ & 23/MIB/2014 & Pig & India & KR149284 & [42] \\
\hline CSFV-a4 & $(2.2)$ & $322 / \mathrm{MIB} / 2013$ & Pig & India & KR350488 & [42] \\
\hline CSFV-a4 & $(2.2)$ & 99/MIB/2014 & Pig & India & KR350483 & {$[42]$} \\
\hline CSFV-a4 & (2) & CSFV-UP-BR-757-09 & Pig & India & KC533785 & Tomar et al., unpublish \\
\hline \multicolumn{2}{|c|}{ CSFV-a4 } & Parambi & Wild boar & India & KT239105 & Chandramohan et al, unpublish \\
\hline \multicolumn{2}{|c|}{ CSFV-a5 } & IND/AS/GHY/G4 & Pig & India & KM362426 & [53] \\
\hline \multicolumn{2}{|c|}{ CSFV-a6 } & Fukuoka/72 & Pig & Japan & AB019150 & {$[43]$} \\
\hline \multicolumn{2}{|c|}{ CSFV-a6 } & Honduras & Pig & Honduras & L42426 & [151] \\
\hline \multicolumn{2}{|c|}{ CSFV-b } & $5440 / 99$ & Sheep & Spain & AY159514 & [70] \\
\hline CSFV-c & (3) & 94.4/IL/94/TWN & Pig & Taiwan & AY646427 & [41] \\
\hline CSFV-c & (3.4) & Kanagawa/74 & Pig & Japan & AB019166 & [43] \\
\hline \multicolumn{2}{|c|}{ CSFV-c } & Okinawa/86 & Pig & Japan & AB019172 & [43] \\
\hline CSFV & & Okinawa-86-2 & Pig & Japan & AB019663 & {$[44]$} \\
\hline CSFV-c & (3) & P97 & Pig & Taiwan & L49347 & Liu, unpublish \\
\hline CSFV & & S171 & Contaminant & China & KF006974 & {$[8]$} \\
\hline CSFV nd & $(2.2)$ & Sch180 & Pig & Germany & JQ411560 & [80] \\
\hline CSFV nd & $(1.3)$ & VRI 4167 & Pig & Malaysia & JQ411570 & [80] \\
\hline
\end{tabular}

Alpine chamois (Rupicapra rupicapra); Bactrian camel (Camelus bactrianus); Black Goat (Capra aegagrus hircus); Buffalo (Bubalus bubalis); Cattle (Bos taurus); Goat (Capra hircus); Human (Homo sapiens); Pig (Sus scrofa domesticus); Pyrenean chamois (Rupicapra pyrenaica); Reindeer (Rangifer tarandus); Serow (Capricornis crispus); Sheep (Ovis aries); Wild boar (Sus scrofa); Yak (Bos grunniens), Zebu (Bos indicus).

sequence was identified from a human pathological sample in Brazil. Seven sequences were CSFV vaccinal strains. Eighty-three strains were contaminants of biological products, mainly detected in fetal bovine serum (FBS) samples for laboratory use, but also cell lines, interferon for human use, vaccines for veterinary 
and human use. Most of the samples were from Japan (n 37). Other contaminats were reported from China, India, Australia, New Zealand, United Kingdom, Italy, Belgium, Netherland, Tunisia, USA, Canada, Mexico, Brazil and Argentina. Sequences were compared to previously evaluated strain, reaching a total number of 1426 observations obtained with PNS procedure (Table 2). Qualitative and quantitative evaluation of genomic sequence divergence, in terms of palindromic nucleotide base pairings variations, has been applied for taxonomical segregation, through the evaluation of relevant secondary structure regions in the 5'-UTR of the viral RNA, the three variable regions, V1, V2 and V3 genomic sequences (Table 3), according to the genotyping based on the PNS method [17] [18]. As described by Deng and Brock [19] and Harasawa [20], the 5'-UTR is characterized by a series of secondary sequence stem-loop structures which are divided into 4 domains: A, B, C and D. The most significant is Domain D which corresponds to the IRES and it covers two thirds of the 5'-UTR sequence from nucleotides 139 to 361 and it is conserved among NADL, Osloss, SD-1, Alfort and Brescia Pestivirus reference strains [19]. The predicted Domain D complex palindromic stem-loop structures D2, D3 and D4 [19], or as recently renamed as IRES domains IIIb, IIId1 and IIId2 [21], correspond to PNS V1, V2 and V3 variable loci, respectively. Within the genus, the palindromic structures identifiable in linear sequences were generally characterized by the absence of nucleotides in position 22 of V1 and V2 locus composed by a constant number of 23 nucleotides.

Table 2. Summary of Pestivirus strains $(\mathrm{n}=1426)$ evaluated according to the Palindromic nucleotide substitution (PNS) method at the 5 ' untranslated region of RNA. ${ }^{\star}$ Tentative species.

\begin{tabular}{|c|c|c|c|}
\hline Species & Number of strains & Host & Geographical origin \\
\hline BVDV-1 & 887 & $\begin{array}{l}\text { Cattle, Alpaca, Alpine chamois, } \\
\text { Bactrian camel, Buffalo, Contaminant, } \\
\text { Deer, Goat, Human, Mousedeer, Pig, } \\
\text { Roe deer, Serow, Sheep, Yak, Zebu. }\end{array}$ & $\begin{array}{l}\text { Argentina, Australia, Austria, Belgium, Brazil, Canada, Chile, China, } \\
\text { Colombia, Denmark, Dominican Republic, Egypt, France, Germany, } \\
\text { India, Ireland, Italy, Japan, Mexico, New Zealand, Poland, Portugal, } \\
\text { Slovakia, South Africa, South Korea, Spain, Sweden, Switzerland, } \\
\text { Tunisia, Turkey, UK, USA }\end{array}$ \\
\hline BVDV-2 & 130 & Cattle, Contaminant, Human, Sheep. & $\begin{array}{l}\text { Argentina, Austria, Belgium, Brazil, Canada, Chile, China, France, } \\
\text { Germany, Italy, Japan, Mexico, Netherland, New Zealand, Portugal, } \\
\text { Slovakia, Spain, Tunisia, UK, USA }\end{array}$ \\
\hline BVDV-3 & 62 & $\begin{array}{l}\text { Cattle, Buffalo, Contaminant, Goat, } \\
\text { Sheep, Zebu. }\end{array}$ & $\begin{array}{l}\text { Australia, Bangladesh, Brazil, Canada, China, India, Italy, Mexico, } \\
\text { Thailand, USA }\end{array}$ \\
\hline BDV & 210 & $\begin{array}{l}\text { Sheep, Alpine chamois, Black goat, } \\
\text { Cattle, Goat, Pig, Pyrenean chamois, } \\
\text { Reindeer, Wisent. }\end{array}$ & $\begin{array}{l}\text { Andorra, Australia, Austria, China, France, Germany, Italy, Japan, } \\
\text { New Zealand, Slovakia, Spain, South Korea, Switzerland, Tunisia, } \\
\text { Turkey, UK, USA }\end{array}$ \\
\hline $\mathrm{BDV}-2^{*}$ & 5 & Sheep, Goat. & Italy \\
\hline Pestivirus I & 2 & Sheep. & Turkey \\
\hline CSFV & 126 & Pig, Cattle, Contaminant, Sheep. & $\begin{array}{l}\text { China, France, Germany, Honduras, India, Italy, Japan, Malaysia, } \\
\text { Netherlands, Poland, Russia, Spain, Switzerland, Taiwan, USA }\end{array}$ \\
\hline Pronghorn & 1 & Pronghorn. & USA \\
\hline Giraffe & 2 & Cattle, Giraffe. & Kenya \\
\hline Bungowannah & 1 & Pig. & Australia \\
\hline
\end{tabular}


Table 3. Palindromic structures V1, V2 and V3 variable loci positions in linear sequences of Pestivirus BVDV-1, BVDV-2, BVDV-3, BDV and CSFV species reference strains for the evaluation according to secondary structure characteristics at the RNA 5'-UTR (PNS method).

\begin{tabular}{ccccc}
\hline \multirow{2}{*}{ Reference strains } & \multicolumn{3}{c}{ Variable loci } & \multirow{2}{*}{ Reference } \\
\cline { 2 - 4 } & V1 & V2 & V3 & \\
\hline BVDV-1 Osloss & $197-235$ & $267-289$ & $293-311$ & {$[143]$} \\
BVDV-1 NADL & $198-236$ & $268-290$ & $294-313$ & {$[103]$} \\
BVDV-2 890 & $75-116$ & $148-170$ & $174-191$ & {$[106]$} \\
BVDV-3 Hobi/Brazil/200/2002 & $37-76$ & $106-128$ & $134-152$ & {$[98]$} \\
BDV X818 & $186-224$ & $256-278$ & $282-297$ & {$[100]$} \\
CSFV Alfort/Tübingen & $187-225$ & $257-279$ & $283-297$ & {$[132]$} \\
\hline
\end{tabular}

The variation of loops in V1 and V3 loci determined difference in size of palindromes. Secondary structures were obtained for the entire 5'-UTR sequence of each strain. Palindromic sequences corresponding to the IRES three variable loci were identified in the predicted secondary structure and considered out of the rest of the nucleotide sequence. Nucleotide sequence secondary structures were predicted according to the algorithm of Zuker and Stiegler [22] using the Genetyx-Mac version 14 program package (Software Development Co., Ltd., Tokyo, Japan). The minimum free energy was calculated by the method of Freier et al. [23]. The PNS software version 2.0 [24], prepared for the application on the genotyping procedures with the keys for Pestivirus identification of genomic sequences, using the $\mathrm{C \#}$ programming language, was also applied for the construction of secondary structure sequence alignment, in order to compute genetic distance among strains. Segregation of Pestivirus species strains into genotypes and relatedness among genotypes within the species was evaluated according to changes in nucleotide base pairs at the level of the secondary palindromic structure of the three variable loci. Genotypes have been defined applying a nomenclature reflecting the level of divergence within the species, and ranked according to increasing divergence expressed in number of divergent base pair (bp), with reference to most common observed sequence combinations in the genus. Genotype roots, base-pair (bp) combinations at the level of low-variable positions (LVP), also defined determinative LVP [14] [17], were identified to support genetic clustering of strain sequences and characterize species genotypes, varying for each species (LVP V1/14, V2/7 and V3/4 for BVDV-1; V1/9, V1/12, V1/16, V1/17 and V1/18 for BVDV-2 or V1/9, V2/1, $\mathrm{V} 2 / 5$ and $\mathrm{V} 3 / 8$ for BDV). Among genotypes, homology was evaluated in terms of shared base pairs in the three variable loci. Cross comparison between types within the genus has been evaluated by computing the divergence percentage, identifying strains showing multiple relation (sequences sharing base pairings specific to different genomic groups, and scoring low divergence values) or borderlines (sequences showing qualitative similarities with a genomic group, but 
with high divergence values, candidates for reallocation as separate groups in the genus), and indicating divergence within groups and among groups quantifying the heterogeneity of a genotype and the genetic distance between groups. Phylogenetic trees based on the 5'-UTR were constructed following sequence alignment of representative species strains with Clustal X [25] by using the neighbour-joining method [26] and visualized with Newick tree format option (Mega version 7.0.26) [27].

\section{Results}

The observation made on the nucleotide sequences of the three variable loci at the level of the 5'-UTR genomic region of Pestivirus strains, according to the PNS genotyping method, allowed the identification of consensus motifs shared by all the Pestivirus species, genus specific base-pairings, and characteristic species and genotype specific PNS, respectively (Table 4). Ten PNS positioned in the V1 and V2 loci were characteristic for the genus. PNS consensus motifs shared by all species in the genus, as the bulge formed by two cytosine nucleotides in the V1 stem position eleven, are presented in Table 4 and schematically shown in Figure 1. Based on the divergence limit value of 9 bp for genotype determination [17], twenty-three genotypes within the BVDV-1 species have been identified, from 1a to 1.23. BVDV-2 accounted for six genotypes, from $2 \mathrm{a}$ to $2 \mathrm{f}$. BDV was clustered into ten genotypes, from a to j. BVDV-3 and CSFV were less heterogeneous with four genotypes.

Secondary sequence construction, efficiently obtained by both available software, Genetyx and PNS [24], revealed a conserved palindromic structure in the genus species (Figure 1). Only in few cases the elaboration of secondary structure resulted slightly problematic. Some difficulties have been encountered due to atypical sequences, showing insertions in the variable loci, as the Chinese BVDV-3 ovine strains HN1559 [KU053497], HN1507 [KU563155], HN1568 [KU053499] and HN1564 [KU053498] (Shi et al., unpublished) with U U, A A, G G or C C bulge insertion in the V1 locus. Similarly, BVDV-3 strains IndMDV18963/12 [KM201318] and IndBHA6604/12 [KM201307] showed deletions in V3 locus positions 5 and 6 . The predicted secondary structures of the three variable loci were aligned for comparison of base pairings in the different positions (Table 5 ). Different base pairing combinations were identified for genotype characterization (BVDV-1 n 416; BVDV-2 n 66; BVDV-3 n 33, BDV n 99 and CSFV n 37), and considered for identification marker definition. Strains showing sequence identity at the level of the three variable loci or sharing non-relevant variations as $\mathrm{G}^{*} \mathrm{U}$ or G-C (G:Y) were excluded (Table 6). The summary of strains clustering into genotypes of Pestivirus species is presented in Table 7. At the species level, the observed taxonomic status of the examined strains corresponded to the estimation obtained by phylogenetic trees constructed from the alignment with the representative strains from the identified genogroups (Figures 2-4). Different species genotypes, showing peculiar genetic 
Table 4. Palindromic nucleotide substitutions (PNS) characteristic to the genus Pestivirus and the BVDV-1, BVDV-2, BVDV-3, BDV, Pestivirus I and CSFV species with related genotypes. The position of base pairings is defined by numbering from the bottom of the variable locus. BVDV-1 genotypes Locus Characteristic PNS markers. Determinative low variable positions (LVP) selected for sequence clustering into genotypes. PP: prevalent position; HVP: highly variable position. $\mathrm{R}=\mathrm{A}$ or $\mathrm{G}$; $\mathrm{Y}=\mathrm{C}$ or $\mathrm{U}$. Genotype name according Vilcek et al. [165] and subsequent authors in parenthesis.

\begin{tabular}{|c|c|c|}
\hline Genus & Locus & Characteristic PNS markers \\
\hline & $\mathrm{V} 1$ & 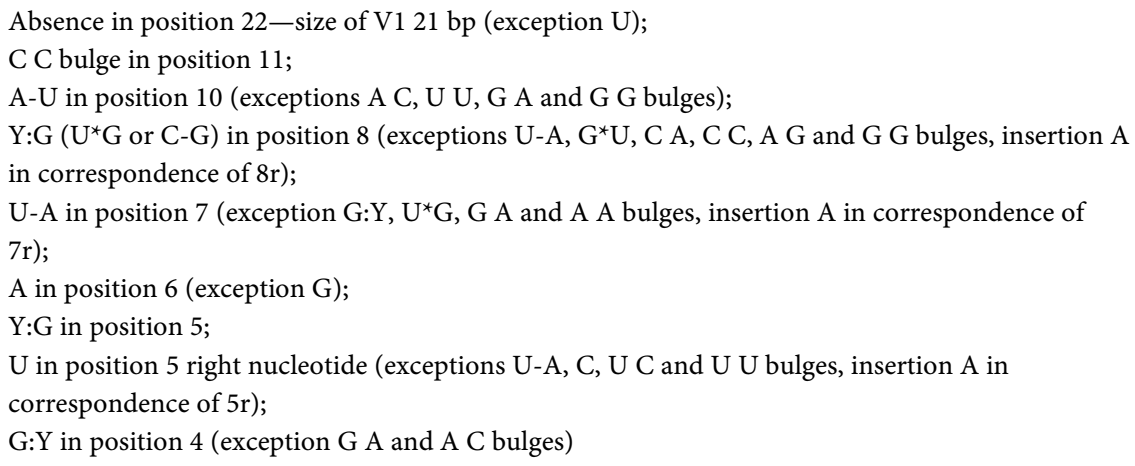 \\
\hline & $\mathrm{V} 2$ & $\begin{array}{l}\text { GGGGY loop (V2 positions 10-12: V2/10 G:Y; V2/11 GG; V2/12 G) (exceptions V2/12: } \\
\text { GG-GGGGGGG-insertion G in V2 loop, absence-GGGY-deletion of G in V2 loop; V2/11: } \\
\text { CG-GCGGY; V2/10: GG-GGGGG, AG-AGGGG, UU-UGGGU); } \\
\text { Y:G in position } 8 \text { (exception A G and C A bulges, insertion A) }\end{array}$ \\
\hline Species & Locus & Characteristic PNS markers \\
\hline \multirow[t]{3}{*}{ BVDV-1 } & $\mathrm{V} 1$ & V1/21 Absence; U-A in position 15 (exception $Y: G-U^{*} G$ or C-G, A A, C A and U U bulges) \\
\hline & $\mathrm{V} 2$ & G:Y in position 5 (exceptions A-U, U-A) \\
\hline & V3 & $\begin{array}{l}\text { G:Y in position } 5 \text { (exception Y:G, G A bulge) } \\
\text { A in position } 10 \text { (exceptions G, U, A-U, U-A, G:Y, C-G or A C, A A, G A, U U, C C and G G } \\
\text { bulges or absence) }\end{array}$ \\
\hline \multirow[t]{3}{*}{ BVDV-2 } & $\mathrm{V} 1$ & $\begin{array}{l}\text { A-U or A C bulge in position } 20 \text { (exceptions G:Y, C C or A A bulges, absence); A, G or U in } \\
\text { position } 21 \text { (exception G G, absence); absence in V1/20 and } 21 \text { is limited to strain A3-118/US is } \\
\text { not considered in software parameters due to confusion with BVDV1 definition }\end{array}$ \\
\hline & $\mathrm{V} 2$ & U-A or Y:G in position 6 (exception C A bulge) \\
\hline & V3 & A-U or A C bulge in position 7 (exception G:Y and AA bulge Chinese strains) \\
\hline \multirow{2}{*}{$\begin{array}{l}\text { BVDV-3 } \\
\text { (HoBi group) }\end{array}$} & $\mathrm{V} 1$ & U-A in position 15 (exception CA BGD/ZS1 and BGD/ZS5) \\
\hline & V3 & $\begin{array}{l}\text { G-C or } G^{\star} U \text { in position } 3 \text { (exception A-U or GA bulge BGDZS1); A-U in position } 7 \text { (exception } \\
\text { G-C or A G, C U, U U and U C bulges); A in position } 10 \text { (exception UA, G) }\end{array}$ \\
\hline \multirow[t]{3}{*}{ BDV } & $\mathrm{V} 1$ & $\begin{array}{l}\mathrm{C}-\mathrm{G} \text { or } \mathrm{U}^{*} \mathrm{G} \text { in position 13; } \mathrm{A}-\mathrm{U} \text { in position } 14 \text { (exceptions } \mathrm{U}-\mathrm{A}, \mathrm{G}: \mathrm{Y} \text { and } \mathrm{A} \text { A bulge); G:Y or A-U } \\
\text { in position } 15 \text { (exceptions } \mathrm{C} \mathrm{U} \text { and A C bulges) }\end{array}$ \\
\hline & $\mathrm{V} 2$ & $\mathrm{U}-\mathrm{A}, \mathrm{Y}: \mathrm{G}$ or $\mathrm{A}-\mathrm{U}$ in position 5 \\
\hline & V3 & $\begin{array}{l}\mathrm{U} \text { C and } \mathrm{U} \text { U bulges or Y:G in position } 7 \text { (exceptions A-U, U-A, G:Y and C C, A C, C U and C A } \\
\text { bulges) }\end{array}$ \\
\hline $\begin{array}{l}\text { BDV-2 (Italian ovine } \\
\text { isolates) tentative } \\
\text { species }\end{array}$ & V1 & $\begin{array}{l}\mathrm{C}-\mathrm{G} \text { or } \mathrm{U}^{*} \mathrm{G} \text { in position } 13 \text {; } \mathrm{U}-\mathrm{A} \text { or } \mathrm{C} \text { A bulge in position } 15 \\
\mathrm{G}: \mathrm{Y} \text { or } \mathrm{G} \text { G bulge in position } 8\end{array}$ \\
\hline \multirow[t]{3}{*}{ Pestivirus I } & $\mathrm{V} 1$ & $\begin{array}{l}\text { C- } \mathrm{G} \text { or } \mathrm{U}^{*} \mathrm{G} \text { in position } 13 \text {; } \mathrm{C}-\mathrm{G} \text { in position } 14 \text {; } \mathrm{G} \text { : } \mathrm{Y} \text { or A-U in position } 15 \text { (exceptions } \mathrm{C} \text { U and A } \\
\text { C bulges) }\end{array}$ \\
\hline & $\mathrm{V} 2$ & G:Y in position 5 \\
\hline & V3 & $\mathrm{Y}$ :G in position 7; $\mathrm{U}$ Uor $\mathrm{C} \mathrm{U}$ bulge in position 10 \\
\hline
\end{tabular}


Continued

\begin{tabular}{|c|c|c|}
\hline \multirow[t]{2}{*}{ CSFV } & V1 & U-A in position 13 (exception $\mathrm{U}^{\star} \mathrm{G}$ or C-G; C A bulge) \\
\hline & V3 & $\mathrm{U}-\mathrm{A}$ in position 2; C A or U-A in position 7; $\mathrm{U}$ or $\mathrm{C}$ in position 8 (exception $\mathrm{A}$ ) \\
\hline \multirow[t]{5}{*}{ BVDV-1 genotypes } & Determinative & Additional markers \\
\hline & LVP (V1/14; & (PP, LVP, HVP) \\
\hline & V2/7; V3/4) & \\
\hline & Genotype & \\
\hline & markers & \\
\hline BVDV-1a & YG, GY, AU & V1/12 GY/AU; V2/5 GC; V2/7 (exception C C bulge) \\
\hline \multirow[t]{4}{*}{ BVDV-1b } & UA, RY, GC & b.1 V1/14 (exception G A bulge) \\
\hline & (b.1V2/7 AU; & V2/7 (exception A C bulge) \\
\hline & b.2V2/7 GY) & $\mathrm{V} 3 / 4$ (exception $\mathrm{AU})$ \\
\hline & & b.2 V1/14 (exception A A; C A and G A bulges) \\
\hline BVDV-1.3 (D) & $\mathrm{CG}, \mathrm{AC}, \mathrm{AU}$ & (V1/14 exception C A and A G bulges) \\
\hline \multirow[t]{3}{*}{ BVDV-1.4 } & CG, AU, GC & V1/12 GY/AU \\
\hline & & V3/6 UA \\
\hline & & V3/4 (exception AU) \\
\hline BVDV-1.5 (I) & CG, GY, GC & $\mathrm{V} 3 / 2 \mathrm{AC}$ \\
\hline \multirow[t]{3}{*}{ BVDV-1.6 $(\mathrm{N}, \mathrm{T})$} & CG, GC, GU & V1/16 AU/UU; \\
\hline & & V2/2 CG; V2/6 GY; \\
\hline & & V3/3 CG; V3/6 UA; V3/8 UC/AC/GC \\
\hline BVDV-1.7 (O) & CG, GY, GC & $\mathrm{V} 2 / 2 \mathrm{UA} ; \mathrm{V} 3 / 9 \mathrm{AU} / \mathrm{AA} / \mathrm{GC}$ \\
\hline \multirow[t]{3}{*}{ BVDV-1.8 (C) } & CG, GC, AU & V1/12 AC (exception C C bulge) \\
\hline & $($ Root $\mathrm{A}$ non $\mathrm{A})$ & $\mathrm{V} 2 / 7$ (exception AU) \\
\hline & & V3/4 (exception AC) \\
\hline BVDV-1.9 (H) & CG, GC, GC & $\mathrm{V} 1 / 20 \mathrm{GA} ; \mathrm{V} 2 / 6 \mathrm{CU} / \mathrm{UU}$ \\
\hline \multirow[t]{2}{*}{ BVDV-1.10 (Q) } & CG, GC, GC & V1/15 AA or CA (exception species marker); V2/2 UA; V2/5 GC; \\
\hline & & $\mathrm{V} 3 / 9 \mathrm{UC} / \mathrm{UU}$ \\
\hline \multirow[t]{4}{*}{ BVDV-1.11 (E) } & CG, GY, GC & V1/17 GA/GG/AG (exception A A bulge and GU); \\
\hline & & V2/6 AU/AC/UU; \\
\hline & & $\mathrm{V} 3 / 6 \mathrm{UA}$ \\
\hline & & V3/8 AA/GA (exception A G bulge) \\
\hline \multirow[t]{4}{*}{ BVDV-1.12 (F, R, S) } & 12.1 & 12.1 \\
\hline & CG, GY, GC & $\mathrm{V} 3 / 6 \mathrm{GC} \mathrm{V} 3 / 8 \mathrm{AU} / \mathrm{AC}$ \\
\hline & 12.2 & 12.2 \\
\hline & CG, AU/GC, GC & V3/6 GA;V2/7 (exception GC) \\
\hline \multirow[t]{2}{*}{ BVDV-1.13 (C, J) } & CG, GY, GC & (V1/14 exception C A bulge); \\
\hline & & V1/16 CR (C-G or C A bulge) V1/17 GA (exception G*U); V2/6 AU (exception A C bulge) \\
\hline BVDV-1.14 (R) & $\begin{array}{l}\text { YG, GY, AU } \\
\text { (Root A non A) }\end{array}$ & V2/5 AU (exception species marker) \\
\hline \multirow[t]{8}{*}{ BVDV-1.15 (M) } & 15.1 & 15.1 \\
\hline & $\mathrm{CG}, \mathrm{GC} / \mathrm{AC}$ & V1/19 GY/GG; V2/2 CG/UA; V2/4 CG/CA (exception G G bulge); V2/5 GC; \\
\hline & GY/AU (Also & V3/9 CC/UC/UU (exception U) V3/4 (exception UA) \\
\hline & Root A non A) & \\
\hline & 15.2 & 15.2 \\
\hline & CG, GC, GY/AU & V1/19 GY/CU; \\
\hline & (Also Root A non & V2/4 CG; V2/5 AU (exception species marker); \\
\hline & A) & $\mathrm{V} 3 / 9 \mathrm{CC} / \mathrm{UC}$ \\
\hline
\end{tabular}


Continued

\begin{tabular}{|c|c|c|}
\hline BVDV-1.16 (L) & CG, GC, GC & V2/6 UA \\
\hline BVDV-1.17 (F) & CG, GC, GC & V3/6 AA (exception G A bulge); V3/8 A A bulge \\
\hline BVDV-1.18 (P) & $\mathrm{CG}, \mathrm{GC} / \mathrm{AC}, \mathrm{GC}$ & $\begin{array}{l}\text { V1/17 AU/CA } \\
\text { V2/6 GC; V3/3 UA; V3/8 AA }\end{array}$ \\
\hline BVDV-1.19 (G) & CG, GY, GC & V1/15 CG; V2/5 AU (exception species marker) \\
\hline \multirow[t]{2}{*}{ BVDV-1.20 (L, X) } & $\begin{array}{l}\text { BVDV-1.20.1 } \\
\text { YG, GY, AU } \\
\text { (Root A non A) }\end{array}$ & $\begin{array}{l}\text { BVDV-1.20.1 } \\
\text { V1/15 CG; V2/5 AU (exception species marker) }\end{array}$ \\
\hline & $\begin{array}{l}\text { BVDV-1.20.2 } \\
\text { YG, GY, GC }\end{array}$ & $\begin{array}{l}\text { BVDV-1.20.2 } \\
\text { V1/15 CA; V2/5 AU (exception species marker) }\end{array}$ \\
\hline BVDV-1.21 (U) & $\begin{array}{l}\text { UA, GC, UA } \\
\text { (origine B, } \\
\text { unique) }\end{array}$ & $\begin{array}{l}\text { BVDV-1.21.1 } \\
\text { V1/15 CG (exception species marker); V3/4 exception G A bulge; V3/5 CG (exception species } \\
\text { marker); V3 loop position } 10 \mathrm{CG} \text { (exception species marker) (exception C C bulge); V3 size longer } \\
\text { (11bp - 21n) (exception 10bp - 20n); V3/2 AU } \\
\text { BVDV-1.21.2 } \\
\text { V3/4 exception G A bulge; V3/5 CG (exception species marker); V3 loop position } 10 \mathrm{CG} \\
\text { (exception species marker) (exception C C bulge); V3 size longer (11 bp - 21n) (exception } 10 \mathrm{bp}- \\
\text { 20n) } \\
\text { V3/2 GU }\end{array}$ \\
\hline BVDV-1.22 (K) & $\begin{array}{l}\text { BVDV-1.22.1 } \\
\text { CG, AC, GC } \\
\text { BVDV-1.22.2 } \\
\text { CG, GC, GC }\end{array}$ & $\begin{array}{l}\text { BVDV-1.22.1 } \\
\text { V2/3 UA; V2/5 GC } \\
\text { BVDV-1.22.2 } \\
\text { V2/3 UA; V2/5 UA (exception species marker) }\end{array}$ \\
\hline BVDV-1.23 (P) & CG, AC, GC & $\mathrm{V} 3 / 5 \mathrm{GA}$ \\
\hline BVDV-2 genotypes & Locus & Characteristic PNS markers \\
\hline BVDV-2a & V1 & C-G in position 16 ; $\mathrm{U}^{\star} \mathrm{G}, \mathrm{C}-\mathrm{G}$ or $\mathrm{U}-\mathrm{A}$ in position 18 (exceptions $\mathrm{U} \mathrm{C}$ and $\mathrm{C}$ A bulges) \\
\hline \multirow[t]{2}{*}{ BVDV-2b } & V1 & $\begin{array}{l}\text { G-C or } \mathrm{G}^{*} \mathrm{U} \text { in position 12; U-A in position } 16 \text { (exception CA bulge); G A, G G or A C bulges or } \\
\mathrm{G}-\mathrm{C} \text { in position 17; G G or G A bulges in position } 18\end{array}$ \\
\hline & V3 & higher V3 loop, $\mathrm{U}$ in position 10 (exceptions $\mathrm{G}^{*} \mathrm{U}, \mathrm{UA}$ and $\mathrm{A}$ ) \\
\hline \multirow[t]{2}{*}{ BVDV-2c } & V1 & U-A in position 8 ; G A bulge in position 17; G A bulge in position 18; $\mathrm{C} \mathrm{C}$ bulge in position 20 \\
\hline & V3 & G-C in position 7 \\
\hline BVDV-2d & V1 & $\begin{array}{l}\text { Shorter V1 loop-size of V1 } 19 \text { bp; U-A in position 15; GA bulge in position 17; G A bulge in } \\
\text { position } 18\end{array}$ \\
\hline \multirow[t]{2}{*}{ BVDV-2e } & V1 & $\begin{array}{l}\text { G-C in position 12; C-G in position } 14 \text {; C-G in position } 16 \text {; } G \text { A bulge in position } 17 \text {; } G \text { A bulge in } \\
\text { position } 18 .\end{array}$ \\
\hline & V3 & higher V3 loop, C in position 10 \\
\hline \multirow[t]{2}{*}{ BVDV-2f } & V1 & $\begin{array}{l}\text { A-U or A C bulge in position 9; A-U in position 12; U-A in position } 16 \text { (exception GA bulge); G A } \\
\text { bulge in position 17; G A bulge in position } 18\end{array}$ \\
\hline & V3 & higher V3 loop, U or U-A in position 10 \\
\hline BVDV-3 genotypes & Locus & Characteristic PNS markers \\
\hline \multirow[t]{3}{*}{ BVDV-3.1 } & V1 & $\begin{array}{l}\text { A-U in position } 1 \text { (exception G:Y, } \mathrm{U} \text { U or } \mathrm{C} \text { U bulges); } \mathrm{Y}: \mathrm{G} \text { in position } 8 \text {; } \mathrm{U}-\mathrm{A} \text { or } \mathrm{C} \text { A bulge in } \\
\text { position 15; A G, A A, U U or G A bulges, A-U or G:Y in position } 18\end{array}$ \\
\hline & $\mathrm{V} 2$ & $\mathrm{Y}: \mathrm{G}$ in position 3 \\
\hline & V3 & G:Y (exception G G bulge) in position 4; Y:G, A G or U-A in position 8 \\
\hline
\end{tabular}




\section{Continued}

BVDV-3.2 V1

BVDV-3.3

BVDV-3.4

BDV-b (2)

BDV-c $(6,8, S W)$

BDV-d (3)

BDV-f $(4,5,7)$

BDV-i

Locus

BDV-g (TU)
G:Y in position 1; A-U in position 8; U-A in position 15; A G bulge in position 18

C A bulge in position 3

A C bulge in position $4 ; \mathrm{Y}: \mathrm{G}$ in position 8

A-U in position 1; Y:G in position 8; C A bulge in position 15; A A bulge in position 18

$\mathrm{Y}: \mathrm{G}$ in position 3

$\mathrm{U}-\mathrm{A}$ in position 4 ; $\mathrm{G}: \mathrm{Y}$ in position 8

$\mathrm{C} C$ bulge in position 1 ; $\mathrm{Y}: \mathrm{G}$ in position 8 ; $\mathrm{U}$-A in position 15 ; $\mathrm{C} \mathrm{U}$ bulge in position 18 $\mathrm{Y}: \mathrm{G}$ in position 3

A-U in position $4 ; \mathrm{Y}: \mathrm{G}$ in position 8

\section{Characteristic PNS markers}

A-U or C U bulge in position 9; A A or A G bulges in position 18 (exception A-U and G G bulge) A-U in position 1; A-U, Y:G or U-A in position 5

A A, G A or A C bulges in position 8 (exceptions G:Y, A)

A-U in position 9 (exception G:Y); $\mathrm{U}-\mathrm{A}, \mathrm{Y}: \mathrm{G}$ in position 16

A-U in position 1; U-A in position 5

$\mathrm{C}$ U bulge in position 1; G:Y or $\mathrm{U}$ U bulge in position 2; A A bulge in position 8 (exception A-U)

G:Y in position 9; A-U, A G or A C bulges in position 16

A-U or A C bulge in position 1; U-A in position 5

A A bulge in position 8

AU in position 3; G:Y in position 9; $\mathrm{A}-\mathrm{U}$ in position 12; $\mathrm{A}-\mathrm{U}$ in position 14; $\mathrm{U}-\mathrm{A}$ in position 16; C A, C U or U U bulges in position 19

$\mathrm{G}: \mathrm{Y}$ in position 1 ; $\mathrm{U}-\mathrm{A}$ in position 5

A G or A A bulges in position 8

G:Y in position 9; G:Y or G G bulge in position 18; G:Y or G G bulge in position 20

G:Y in position 1; Y:G in position 5 (shared with A only)

$\mathrm{U}-\mathrm{A}$ or $\mathrm{C} A$ bulge in position 8

G:Y in position 9 (exception A-U); A-U in position 16 (exception $\mathrm{G}^{\star} \mathrm{U}$ ); Y:G, G:Y or G G bulge in position 18 (exception A G bulge)

G:Y in position 1 (exceptions A-U and C U or G G bulges); Y:G in position 5 (shared with A only)

$\mathrm{U}-\mathrm{A}, \mathrm{Y}: \mathrm{G}, \mathrm{A}$ A or $\mathrm{C}$ A bulges in position 8 (exception $\mathrm{U} \mathrm{C}$ bulge)

G:Y or A-U in position 9

A-U in position 1; A-U in position 5 (exception $\mathrm{G}: \mathrm{Y}$ )

U-A in position 2 (g1 V3/2 CG-strains TU ITA sicily-Shared only with v60-bd-b); Y:G in position 7 (exception $\mathrm{U}-\mathrm{A}$ - shared with al and $\mathrm{e} 1$ ); $\mathrm{U}$ or $\mathrm{C}$ in position 8

$\mathrm{AU}$ in position 3; G:Y in position 9; $\mathrm{U}-\mathrm{A}$ in position 12; $\mathrm{A}-\mathrm{U}$ in position 14; $\mathrm{U}-\mathrm{A}$ in position 16; CC in position 19

G:Y or A-U in position 1; U-A in position 5

$\mathrm{G}: \mathrm{Y}$ in position 4; A-U or A A bulge in position 8

V1/8 CC/AG; G:Y in position 9 (exception GA bulge); Y:G in position 16; A-U or A G bulge in position 19 


\section{Continued}

\begin{tabular}{|c|c|c|}
\hline & $\mathrm{V} 2$ & A-U in position 1; U-A in position 5 \\
\hline & V3 & G: $\mathrm{Y}$ in position 4 ; $\mathrm{A} A$ or $\mathrm{C}$ A bulges in position 8. \\
\hline \multirow[t]{3}{*}{$B D V-j(3)$} & V1 & $\begin{array}{l}\text { G:Y in position } 3 \text { (exception A-U); G:Y in position 9; G:Y in position } 14 \text { (exception A-U); U-A or } \\
Y: G \text { in position 16; C C, U C bulges, U-A or Y:G in position } 19\end{array}$ \\
\hline & $\mathrm{V} 2$ & A-U in position 1; U-A in position 5 \\
\hline & V3 & G:Y in position 4; Y:G, G:Y, U C or A A bulges in position 8 \\
\hline BDV sub-genotypes & Locus & Characteristic PNS markers \\
\hline BDV-a1 (1) & V1 & $\begin{array}{l}\mathrm{U} \text { right nucleotide in position 7; A-U in position 12; } \mathrm{A}-\mathrm{U} \text { or } \mathrm{CU} \text { in position 15; } \mathrm{AA} \text { or } \mathrm{AG} \text { in } \\
\text { position } 18\end{array}$ \\
\hline BDV-a2 (1) & V1 & $\begin{array}{l}\text { G right nucleotide in position 7; G:Y or CC in posizion } 12 \text {; G:Y in position } 15 ; A G \text { or GG in } \\
\text { position } 18\end{array}$ \\
\hline BDV-a3 (1) & V1 & A right nucleotide in position 7; G:Y in position 12; AC in position 15; AG in position 18 \\
\hline BDV-a4 (1) & V1 & G right nucleotide in position 7; G:Y in posizion 12; G:Y in position 15; A-U in position 18 \\
\hline \multirow[t]{3}{*}{ BDV-c1.1 (Sw) } & V1 & A-U in position 16 \\
\hline & $\mathrm{V} 2$ & A-U in position 1 \\
\hline & V3 & G:Y in position 4 \\
\hline \multirow[t]{3}{*}{ BDV-c1.2 (8) } & V1 & A-U in position 16 \\
\hline & $\mathrm{V} 2$ & A-U in position 1 \\
\hline & V3 & A-U in position 4 \\
\hline \multirow[t]{3}{*}{ BDV-c1.3 (8) } & V1 & A C bulge in position 16; $\mathrm{G}: \mathrm{Y}$ in position 21 \\
\hline & $\mathrm{V} 2$ & A- $U$ in position 1 \\
\hline & V3 & G:Y in position 4 \\
\hline \multirow[t]{3}{*}{ BDV-c2 (6) } & V1 & G:Y or U C and U U bulges in position 20; $\mathrm{U}$ or $\mathrm{U}$ U bulge in position 21 \\
\hline & $\mathrm{V} 2$ & A C bulge in position 1 \\
\hline & V3 & C C bulge in position 7 (exception U C bulge) \\
\hline \multirow[t]{2}{*}{ BDV-d1 (3) } & V1 & CU or UU in position 19 \\
\hline & V3 & A-U in position 4; A G bulge in position 8 \\
\hline \multirow[t]{2}{*}{ BDV-d2 (3) } & V1 & CA in position 19 \\
\hline & V3 & G:Y in position 4; A A bulge in position 8 \\
\hline \multirow[t]{2}{*}{ BDV-e1 (4) } & V1 & G:Y in position 16 \\
\hline & V3 & U-A in position 8 \\
\hline \multirow[t]{2}{*}{ BDV-e2 (4) } & V1 & A-U in position 16 ; $G$ in position 21 \\
\hline & V3 & CA in position 8 \\
\hline \multirow[t]{3}{*}{ BDV-f1 $(4,5)$} & V1 & G right nucleotide in position 7; CU in position 17; GG in position 18 \\
\hline & $\mathrm{V} 2$ & G:Y or $\mathrm{Y}: \mathrm{G}$ in position 2 \\
\hline & V3 & UU or UC in position 7 \\
\hline \multirow[t]{3}{*}{ BDV-f2 (4) } & V1 & U right nucleotide in position 7; $\mathrm{Y}: \mathrm{G}$ in position 17 ; G:Y in position 18 \\
\hline & $\mathrm{V} 2$ & UA in position 2 \\
\hline & V3 & A-U or AC in position 7 \\
\hline
\end{tabular}




\section{Continued}

BDV-f3 (4)

V1

V2

V3

BDV-f4 $(4,7)$

V1

V2

V3

BDV-g1 (TU)

V1

V2

V3

BDV-g2 (TU)

V1

V2

V3

BDV-i1

V1

V3

BDV-i2

BDV-j1 (3)

BDV-j2 (3)

CSFV genotypes

CSFV-a

CSFV-c

CSFV-d

V1

V2

V3

CSFV genotype variants Locus

CSFV-a1

G right nucleotide in position 7; CU or A-U in position 17; G:Y, GG or AG in position 18

$\mathrm{UA}$ in position 2

UU or UC in position 7

G right nucleotide in position 7; $\mathrm{CU}$ or $\mathrm{CC}$ in position 17; Y:G in position 18

$\mathrm{Y}: \mathrm{G}$ or $\mathrm{C} \mathrm{U}$ bulge in position 2

$\mathrm{U} \mathrm{U}$ or $\mathrm{U} \mathrm{C}$ bulges in position 7

G:Y in position 1

$\mathrm{U}-\mathrm{A}, \mathrm{C}-\mathrm{G}$ or $\mathrm{U}^{\star} \mathrm{G}$ in position 3

A-U or CU in position 6 (CU all strains from Sicily; shared only with B V60 and D2 AV only)

A-U in position 1

A-U or AC in position 3

AC, UU or CC in position 6

$\mathrm{C}$ C bulge in position 8; A-U in position 19

C A bulge in position 8

A G bulge in position 8; A G bulge in position 19

A A bulge in position 8

A-U in position 3; A-U in position 14; $\mathrm{CC}$ in position 19

$\mathrm{Y}: \mathrm{G}$ in position 8

G:Y in position 3; GC in position 14; UA or UG in position 19

G:Y, U C or A A bulges in position 8

G:Y in position 3; GC in position 14; UC in position 19

$\mathrm{Y}: \mathrm{G}$ in position $8 ; \mathrm{V} 3 / 9 \mathrm{~A}$

\section{Characteristic PNS markers}

A C bulge in position 15 (exception G:Y and A-U)

U-A in position 5; G:Y in position 7

A-U in position 1 (exception A G bulge)

G:Y in position 15; A G bulge in position 19; U-A in position 20

A-U in position 5; A C bulge in position 7

A-U in position 1; $\mathrm{U} C$ bulge in position 6

A-U in position 15

U-A in position 5; G:Y in position 7

A G bulge in position 1

A C bulge in position 15

U-A in position 5; G: $Y$ in position 7

$\mathrm{U} \mathrm{U}$ bulge in position 1

\section{Characteristic PNS markers}

$\mathrm{AC}$ in position 15

A-U in position 1; A-U in position 6 
Continued

\begin{tabular}{|c|c|c|}
\hline & V3 & $\mathrm{C}$ or $\mathrm{U}$ in position 8 \\
\hline \multirow[t]{3}{*}{ CSFV-a2 } & V1 & AC in position 15 (exception G-C) \\
\hline & $\mathrm{V} 2$ & A-U in position 1; G:Y in position 6 \\
\hline & V3 & $\mathrm{A}-\mathrm{U}$ in position 6 ; $\mathrm{U}$ or $\mathrm{C}$ in position 8 \\
\hline \multirow[t]{3}{*}{ CSFV-a3 } & V1 & $\mathrm{AC}$ in position 15 \\
\hline & $\mathrm{V} 2$ & AC in position 1; G:Y in position 6 \\
\hline & V3 & A in position 8 \\
\hline \multirow[t]{3}{*}{ CSFV-a4 } & V1 & G:Y in position 15 \\
\hline & $\mathrm{V} 2$ & A-U in position 1; G:Y in position 6 \\
\hline & V3 & AC or UC in position 6; A in position 8 (exception $\mathrm{C}$ ) \\
\hline \multirow[t]{3}{*}{ CSFV-a5 } & V1 & A-U in position 15 \\
\hline & $\mathrm{V} 2$ & $\mathrm{AC}$ in position $1 ; \mathrm{A}-\mathrm{U}$ in position 6 \\
\hline & V3 & $\mathrm{U}$ in position 8 \\
\hline \multirow[t]{3}{*}{ CSFV-a6 } & V1 & G:Y in position 15 \\
\hline & $\mathrm{V} 2$ & A-U in position 1; A-U in position 6 \\
\hline & V3 & $\mathrm{C}$ in position 8 \\
\hline
\end{tabular}

characteristics, were restricted to specific geographic areas as in Turkey, China and other Asian countries (Figure 5).

The BVDV-1 species was heterogeneous. Some Asian strains showed exception at the level of BVDV-1 species markers. For example, Chinese strains of the genotype BVDV-1.10 showed divergence in V1/15 with A A or C A bulges, instead of species marker U-A pairing. In sub genotype 1.15.2, all strains showed species marker exception in V2/5, with A-U instead of G-C. Similarly, strain S153 [8], genotype 1.23, showed an atypical G A bulge at the level of species marker position in $\mathrm{V} 3 / 5$. The Asian clusters 1.8 and 1.15 shared a root characteristic of genotype BVDV-1a (V1/14 C-G, V2/7 G-C and V3/4 A-U). All the strains belonging to the group 1.8 showed an A C bulge in position 12 in V1 locus, a base pairing not present in any member in the genotype 1a. In this group have been included bovine strains from Australia, China and Japan as Bega (Mackintosh et al., unpublished), Shitara/01/05 [28], Manasi [9], the Australian contaminant strain A1-114/AU [29] and the contaminant strain CRFK reported from Japan [30]. Among Chinese strains of the genotype 1.15, reported as genotype $M$ [2] [7], root A was present in only the strain LZ05 of the sub genotype 15.1. In the sub genotype 15.2, characterized also by an exception at the level of species marker in V2/5 (A-U instead of G-C), root A was present in the majority of the strains. The genotype 1.21 showed a new and atypical U-A/G-C/U-A root associated to the genotype b (sub genotype 2). BVDV-1b, characterized by a variation of base pairing at the level of position 7 in V2 (b.1 V2/7 A-U; b.2 V2/7 $G^{*} U$ or G-C), was the only other BVDV-1 genotype showing $U-A$ pairing in LVP V1/14. The V1/14 U-A was shared exclusively with BVDV-1b and BVDV-1.21 
(A)

(B)

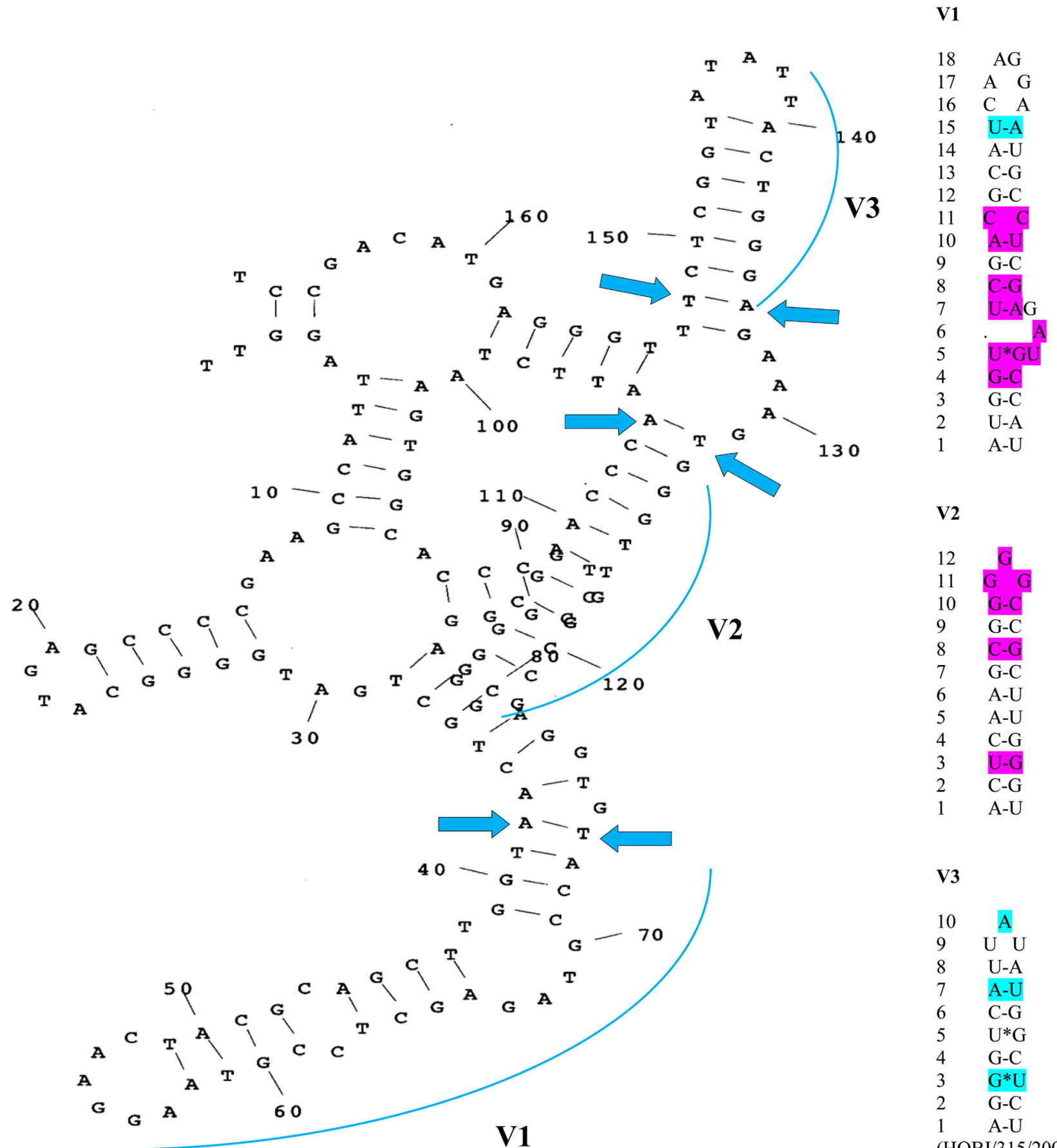

Figure 1. Secondary structure of the entire 5'-UTR sequence of BVDV-3 strain Hobi/315/2004 [98] (a) predicted according to the algorithm of Zuker and Stiegler [22], using the Genetyx-Mac version 10.1 program package (Software Development Co., Ltd., Tokyo, Japan). The minimum free energy was calculated by the method of Freier et al. [23]. Minimum free energy: -88.51 $\mathrm{Kcal} / \mathrm{mol}$. V1-V3 palindromic loci are identified in the 5'-UTR sequence. Variable loci starting base pairs (position 1) referred to PNS procedure are indicated by blue arrows. (b): Schematic presentation of V1-V3 palindromic loci in the 5'-UTR of the tentative species BVDV-3 strain Hobi/315/2004. Base pairings characteristic to the genus (PNS genus specific) are shown in violet. The characteristic base pairings of the species BVDV-3 (PNS species specific) are represented in blue. Distance of V3 from V2: 5 nucleotides. The position of base-pairings is defined by numbering from the bottom of the secondary structures. Watson-Crick base pairings are indicated by a dash (-); tolerated pairings in secondary structure are indicated by an asterisk $\left(^{*}\right)$. 


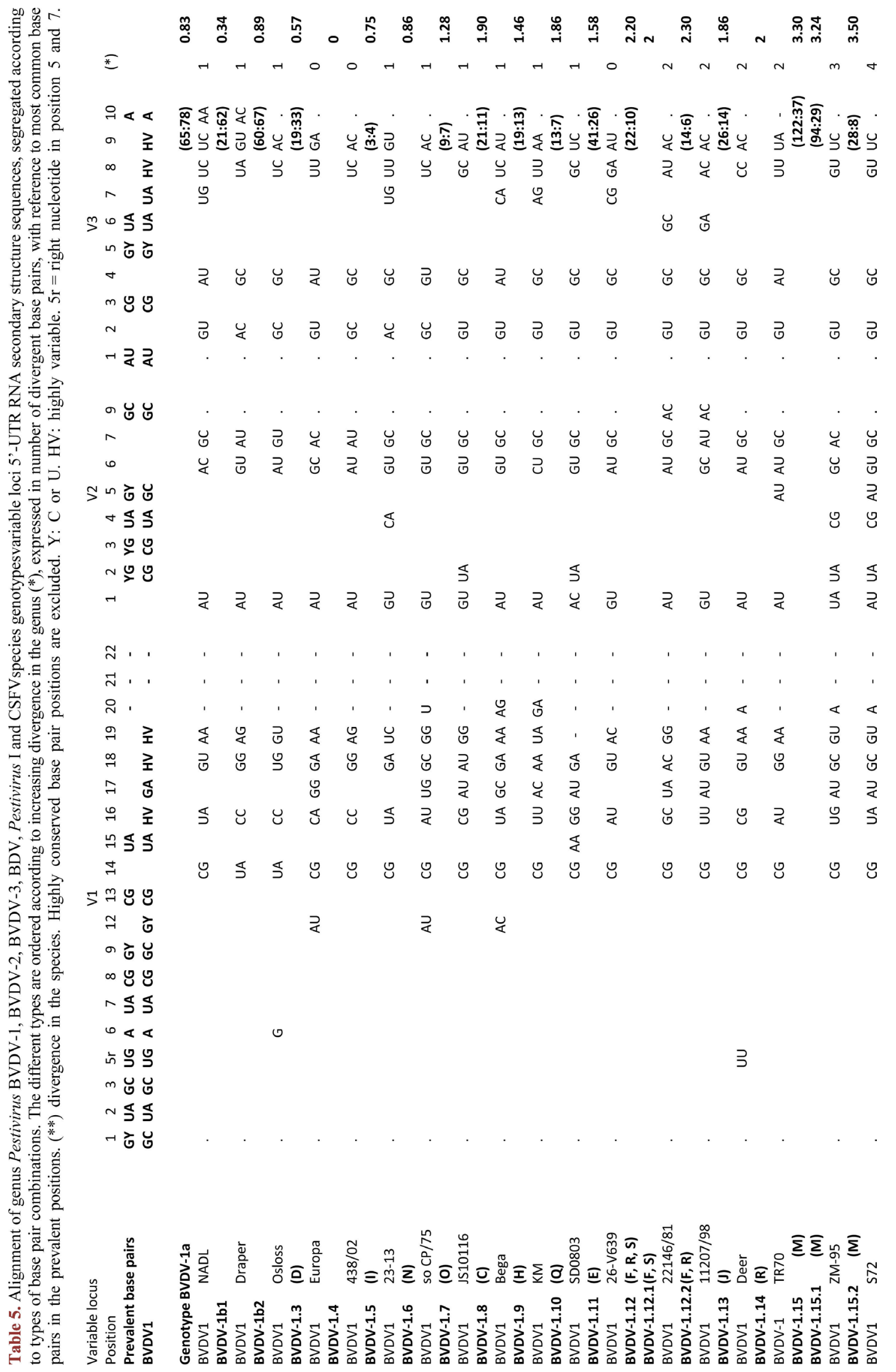




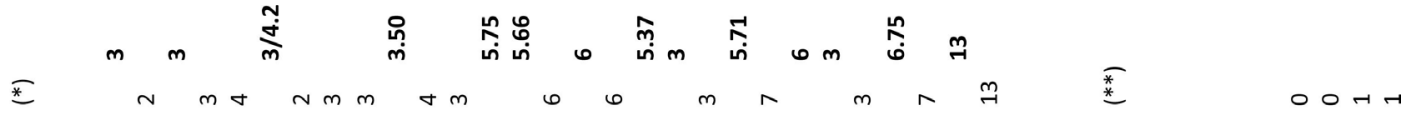

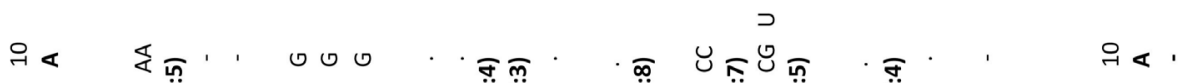

$$
\begin{aligned}
& \text { の } \\
& \infty \text { 至 }
\end{aligned}
$$

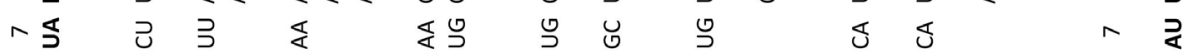

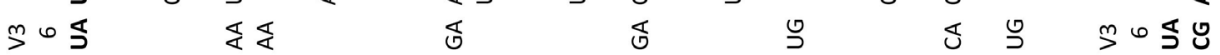

$$
\begin{aligned}
& \text { 的它 } \\
& \text { - } \quad \forall \text { O }
\end{aligned}
$$

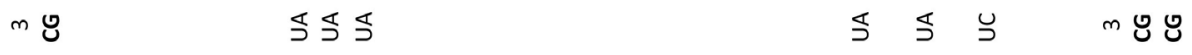

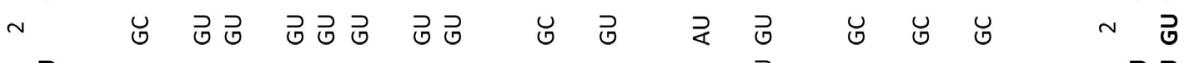

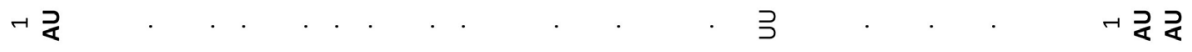

$$
\begin{aligned}
& \text { の } \\
& \wedge \quad \text { S }
\end{aligned}
$$

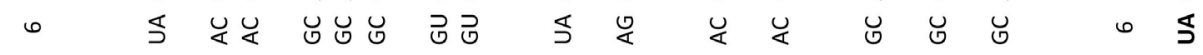

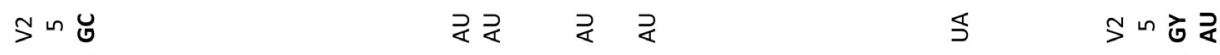

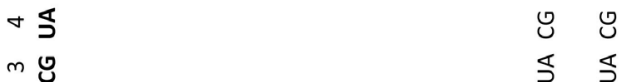

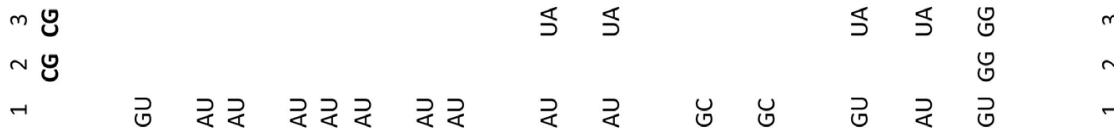

$$
\begin{aligned}
& \approx,, 1,1,1,1, \ldots, \ldots \\
& \text { ন! , , , , , , , , , , , , , , , }
\end{aligned}
$$

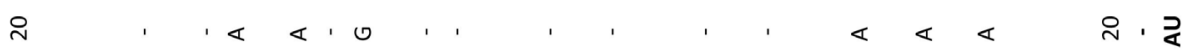

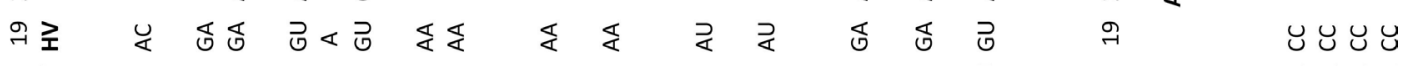

$$
\begin{aligned}
& \text { 冓至 向 } \\
& \text { न }
\end{aligned}
$$

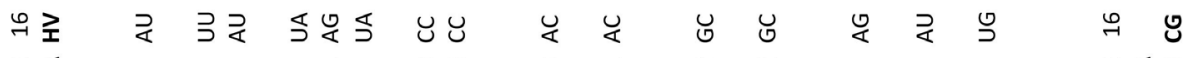

$$
\begin{aligned}
& \text { 年 }
\end{aligned}
$$

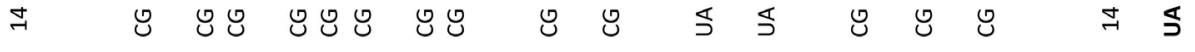

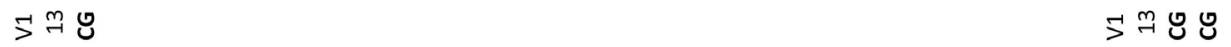

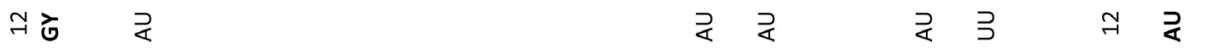

$$
\begin{aligned}
& \text { の } \\
& \infty \text { 艺舟要 } \\
& \wedge \leq \\
& 0<\text { o } \\
& \text { in } \\
& m \text { 品 } \\
& \sim S \\
& \rightarrow \text { O } \\
& 0
\end{aligned}
$$

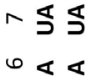

$$
\begin{aligned}
& \text { in }
\end{aligned}
$$

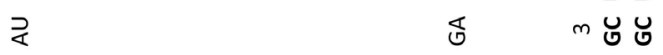

$$
\begin{aligned}
& \sim S \\
& \text { けむを . . }
\end{aligned}
$$

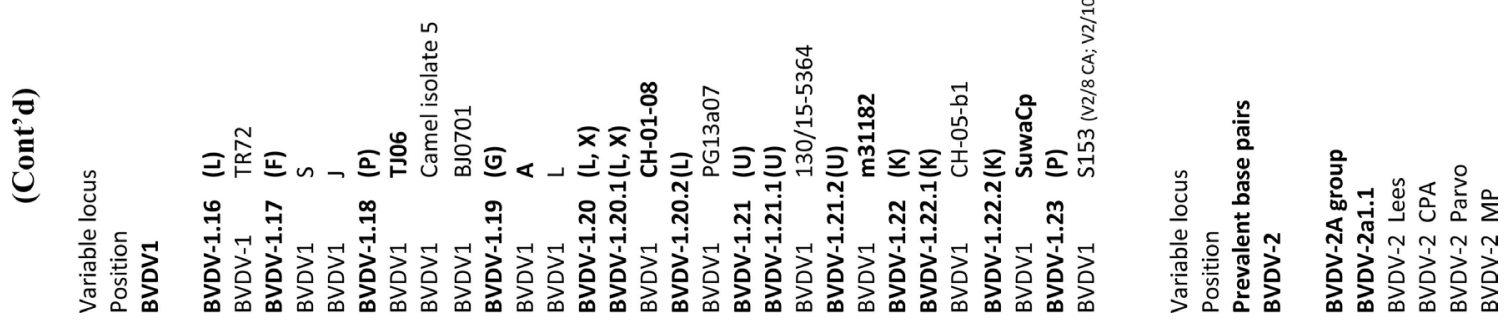




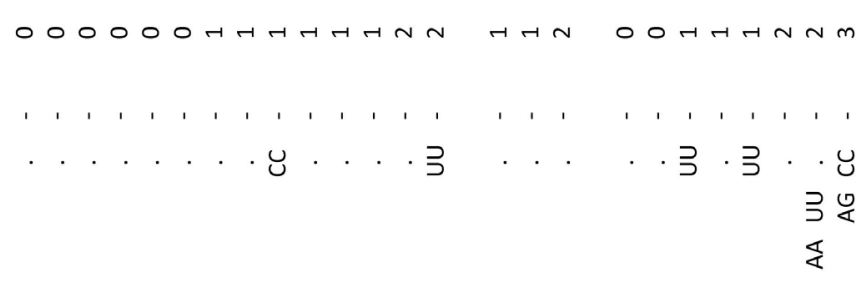

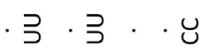

$$
\begin{aligned}
& 8
\end{aligned}
$$$$
\text { 引원 }
$$

$\sim \mathcal{O}$

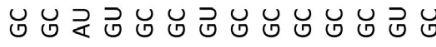

๖ ๖ ৩

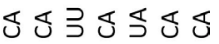

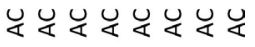

$\rightarrow \frac{2}{4}$

ब Э

$\wedge \mathrm{U}$

○吉 s $\frac{2}{4}$

$\sim 8$

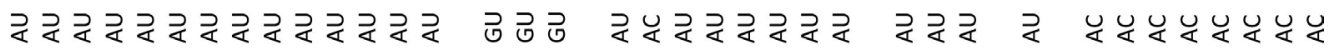
ส

\section{उ}

פ כ פ

จ จ จ

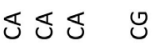

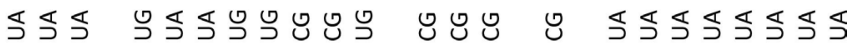

ns

$6 \varangle$

in

$m$ 过

$\sim S$

$\rightarrow \frac{1}{4}$ 

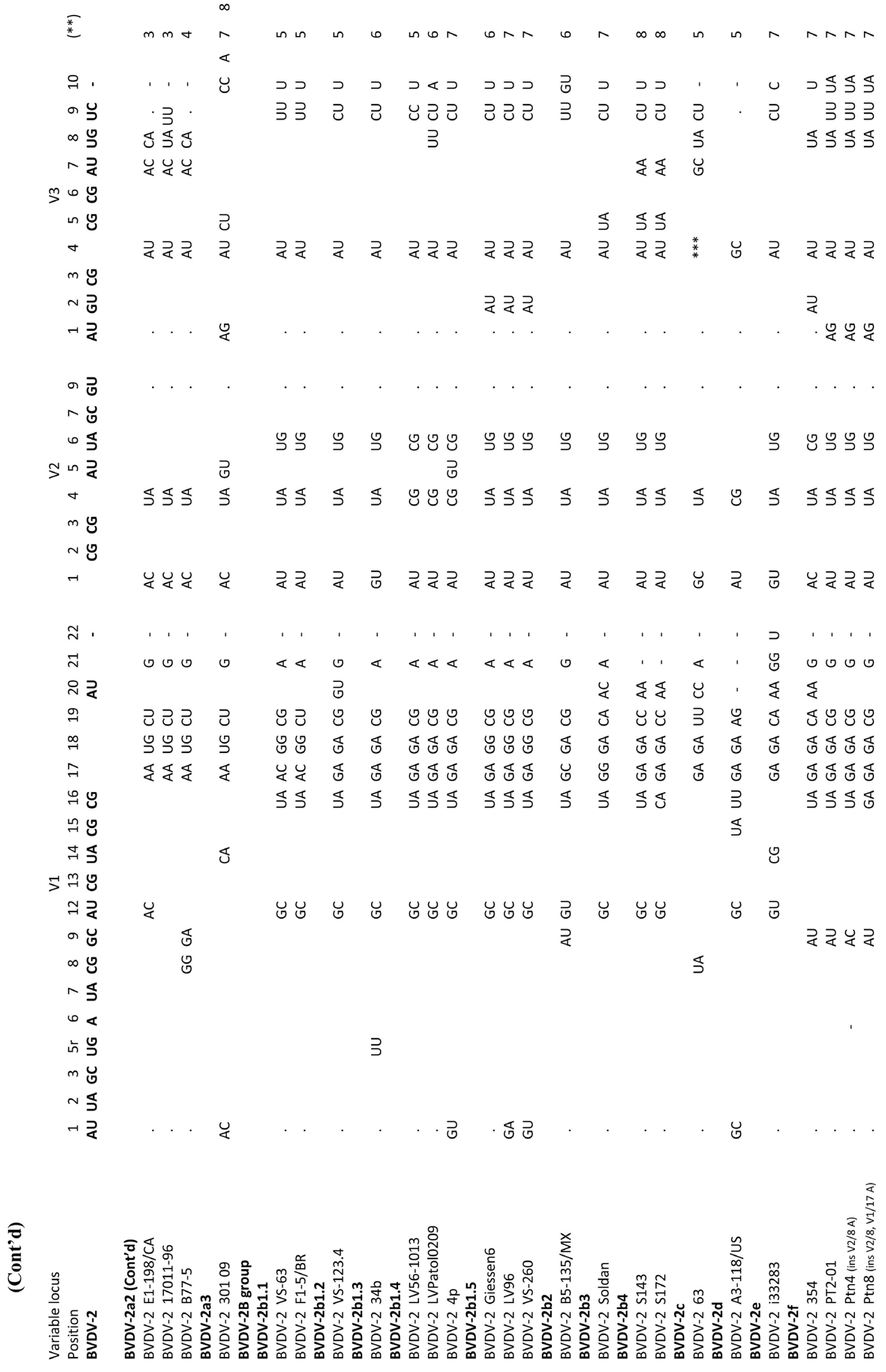


$$
\stackrel{\text { F }}{*}
$$

$\rho<4$

a 引

$\infty$

ᄀ

m०S

๒むす

-

m

$\sim \circlearrowright$

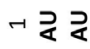

の

○ 仓

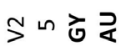

+ 58

mश

$\sim \mathscr{Y}$

- $\overrightarrow{4}$

ก.

ก.

ㅇ.

가

$\rightarrow$

A \&

워

노

त ₹

ᄀ

$\approx$ ¿

のむし

$\infty$ ษ

กSS

$0<4$

旃星

n

NSS

ㅎる

弓

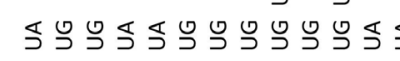
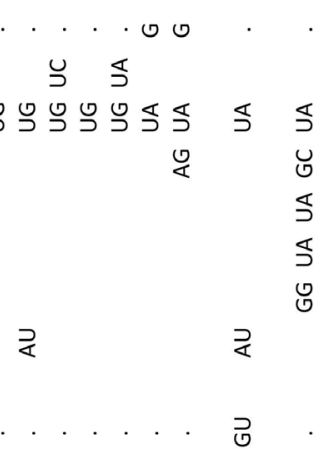

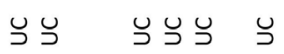

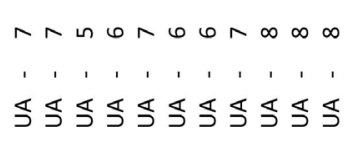

$\wedge \infty$ 구

, '

1.' 1

૫

บ บ บ

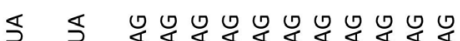

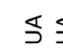

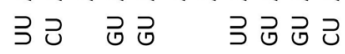

5

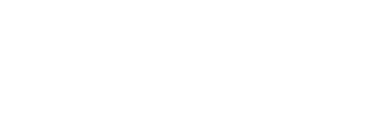

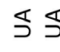

D)

8

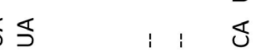

نं ن̀

过是吉层 ऽ

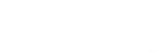

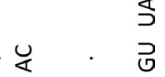

ज

๖ ๖

๖ ๖

ษ

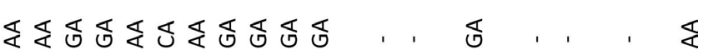

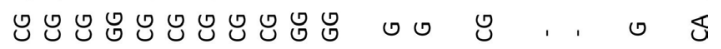

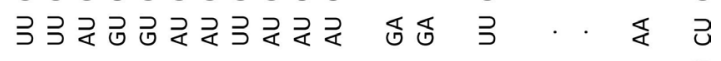

连连

$\unlhd$

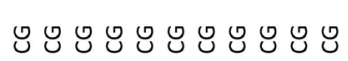

건

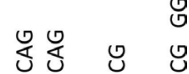

S
定

$\varangle$

$\gtrless \gtrless$

\&芯志

군

ว. ๖ ๖

·

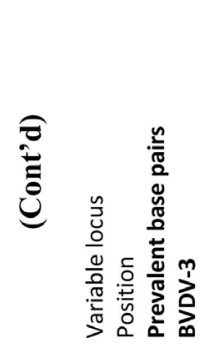

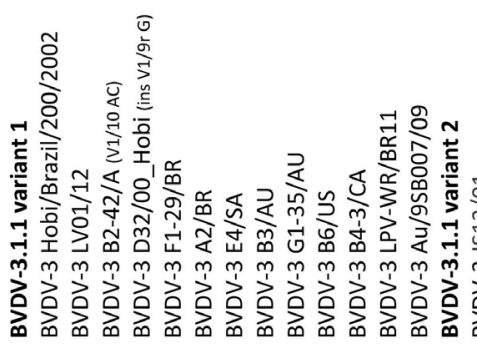

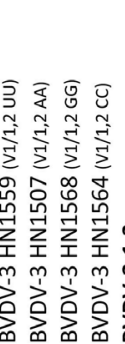

空空

离

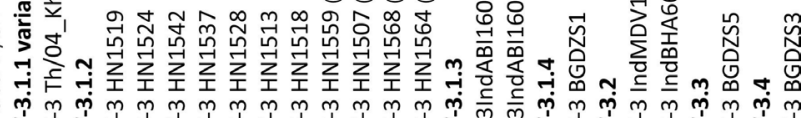

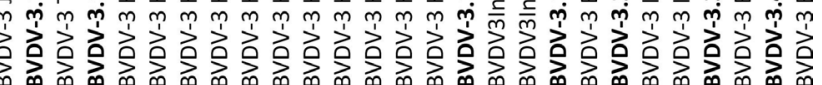




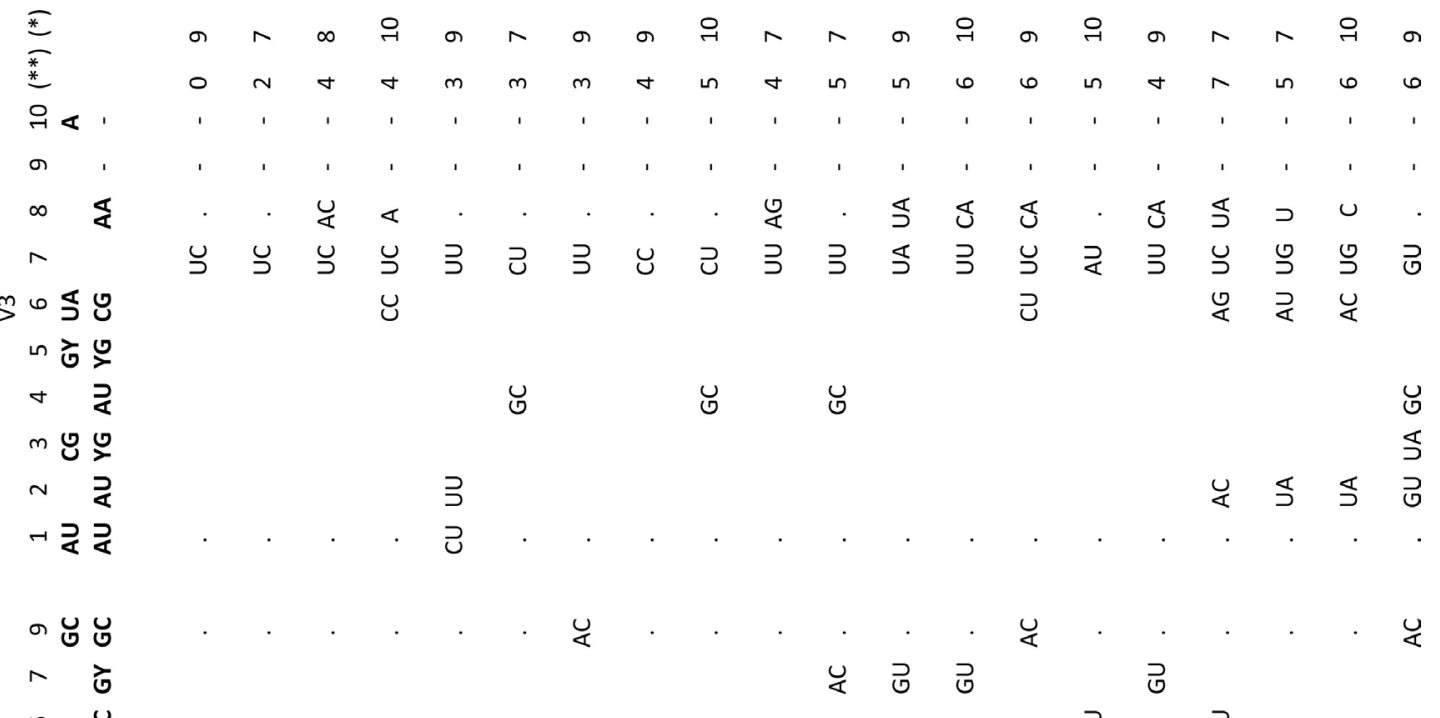

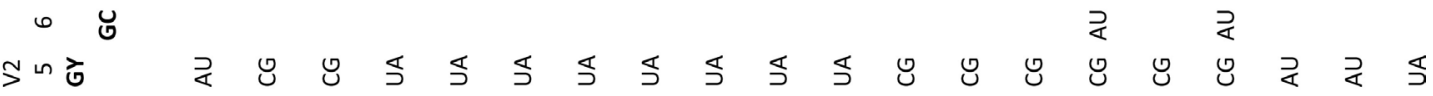

$+\leq \mathbb{8}$

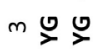

$\sim \supsetneq ⿻$ s 5

S

$\rightarrow \quad z$

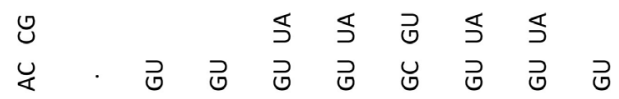

ส

4.

ㅇ.

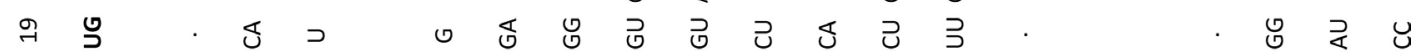
の A

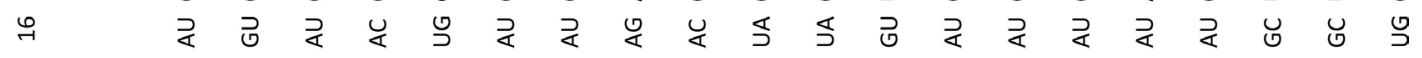
川 स $\overrightarrow{8}$

$>\stackrel{\rightarrow}{*} 8$

ป

のうる ⿸尸

$\infty$ ㅇ 요

几S

$0 \ll \varangle \quad \unlhd$

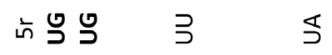

m过

นS马

けむう

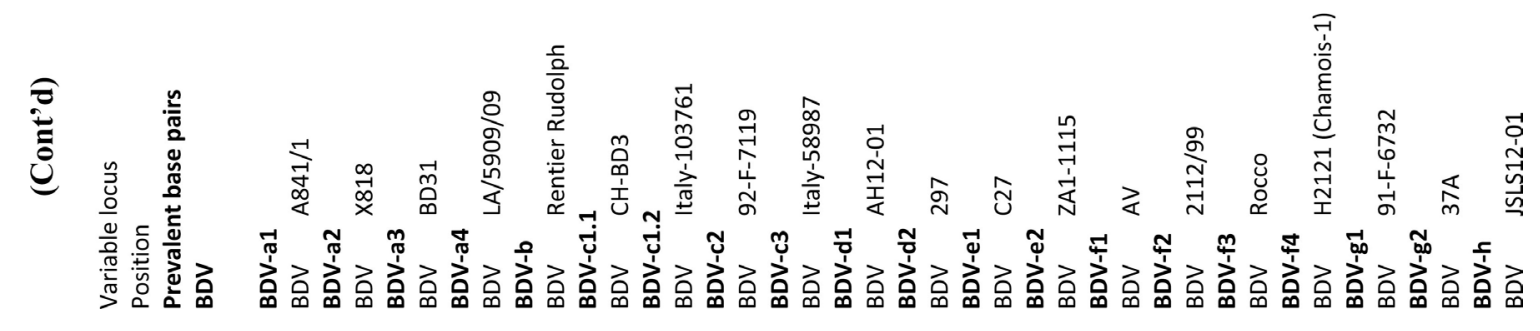




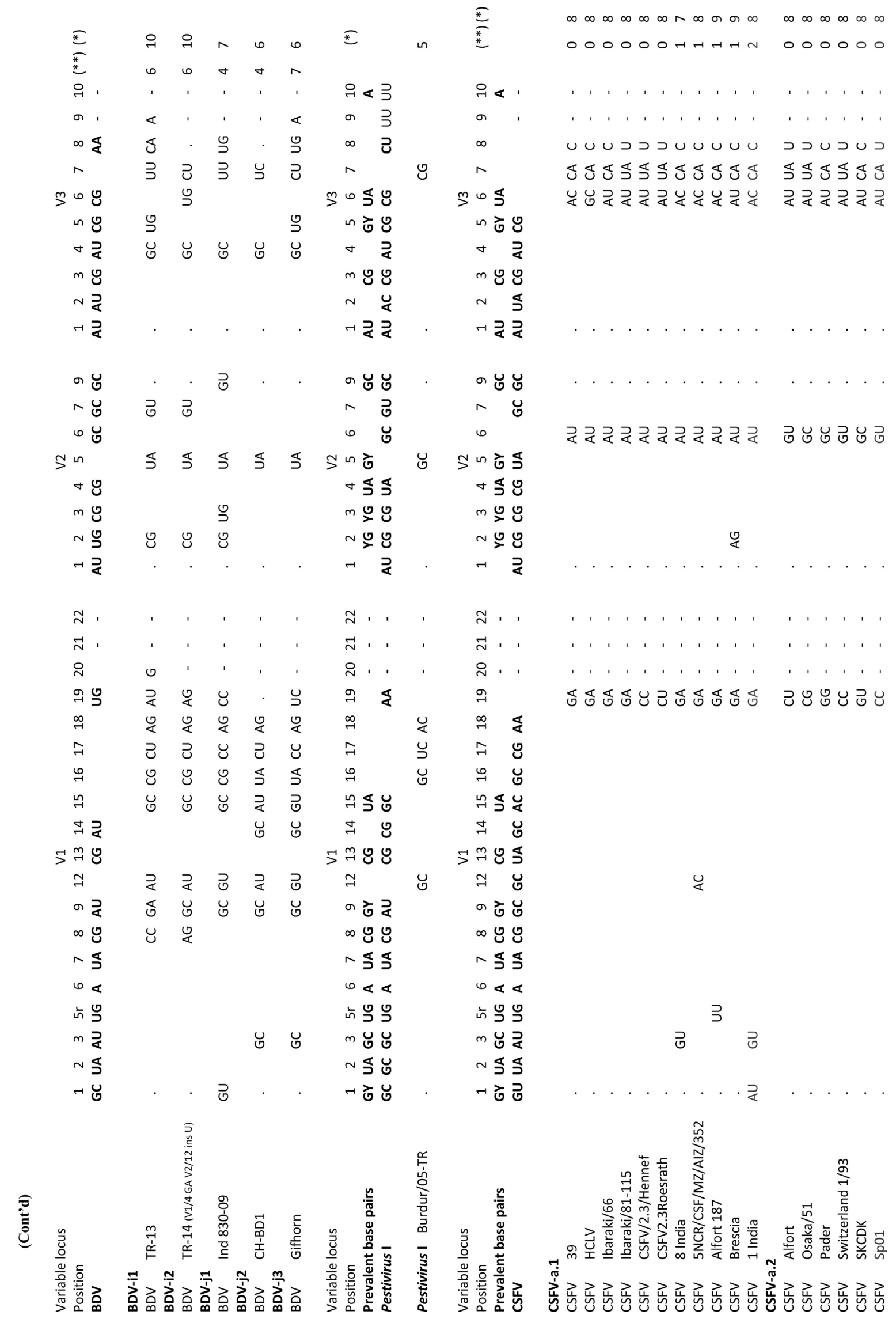


$\stackrel{\text { * }}{\stackrel{*}{*}}$

$$
\text { 윶. }
$$

$\infty$

人

m $0 \leqq$

เ ษ ত

-

n ษ

$\sim \leq$

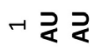

a ড

$\wedge \quad$ S

6

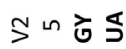

$\checkmark \leq \mathbb{8}$

$m \searrow$

$\sim \searrow$

$\rightarrow \quad \overrightarrow{\&}$

N, ,

ㄱ. ,

이, ,

각

$\stackrel{\infty}{\rightarrow} \varangle$

긍

$\rightarrow$ U

는

ন

ร 껀

ㄱ

எ ঊ Ч

$\infty$ U

$\wedge \leftrightarrows 5$

$0<4$

เท่

$m$ 过

N【

ーむ

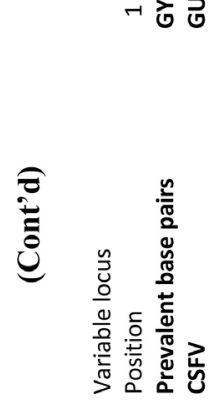

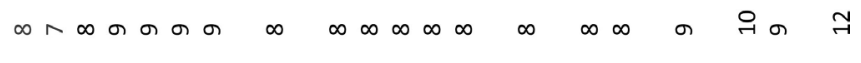

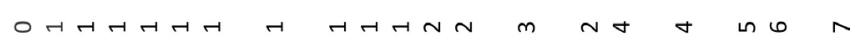

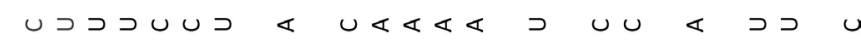

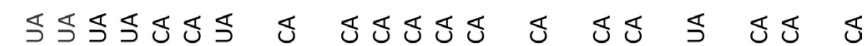

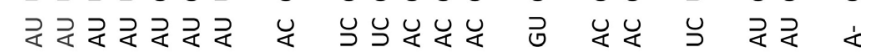

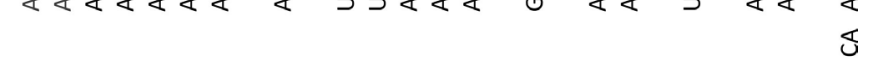
¿ $\dot{u}$

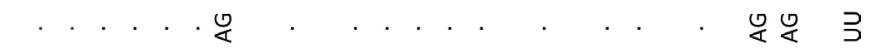

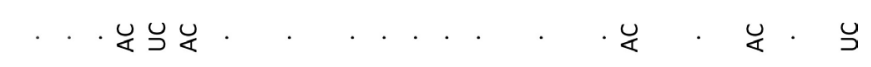

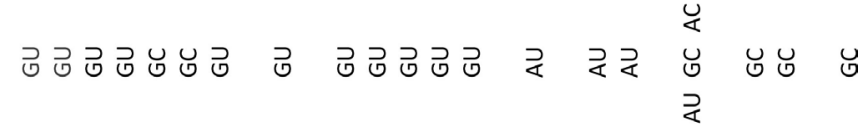
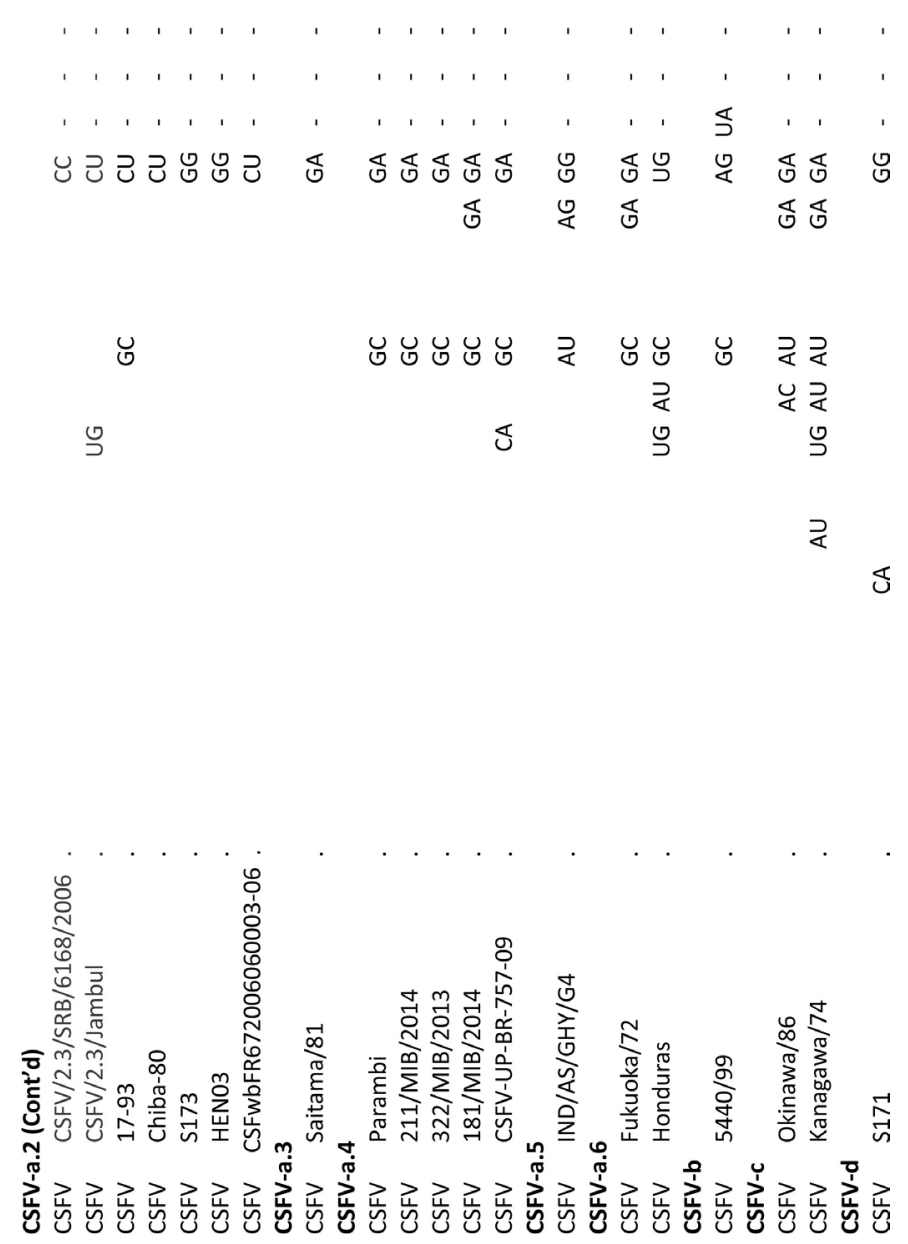


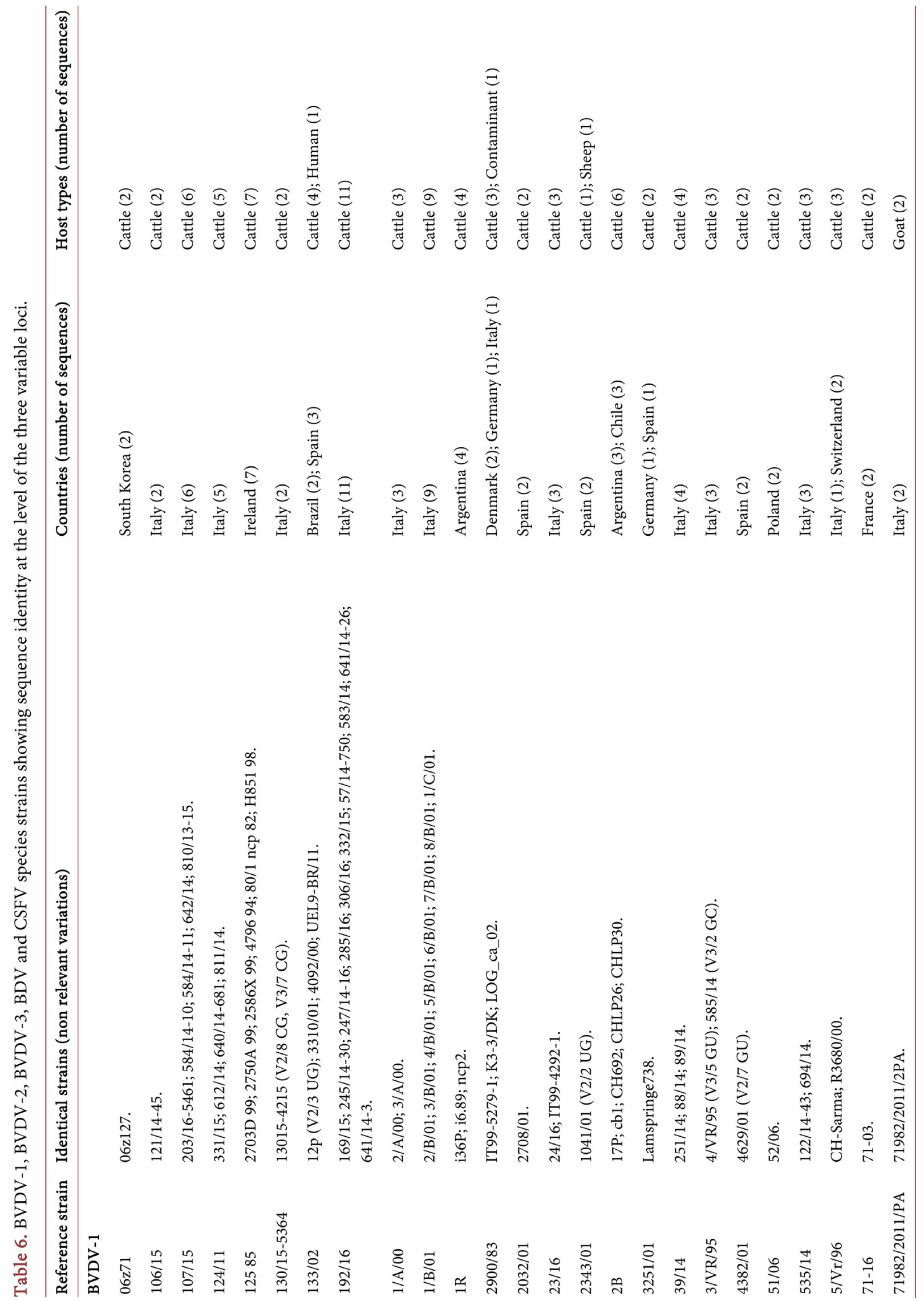




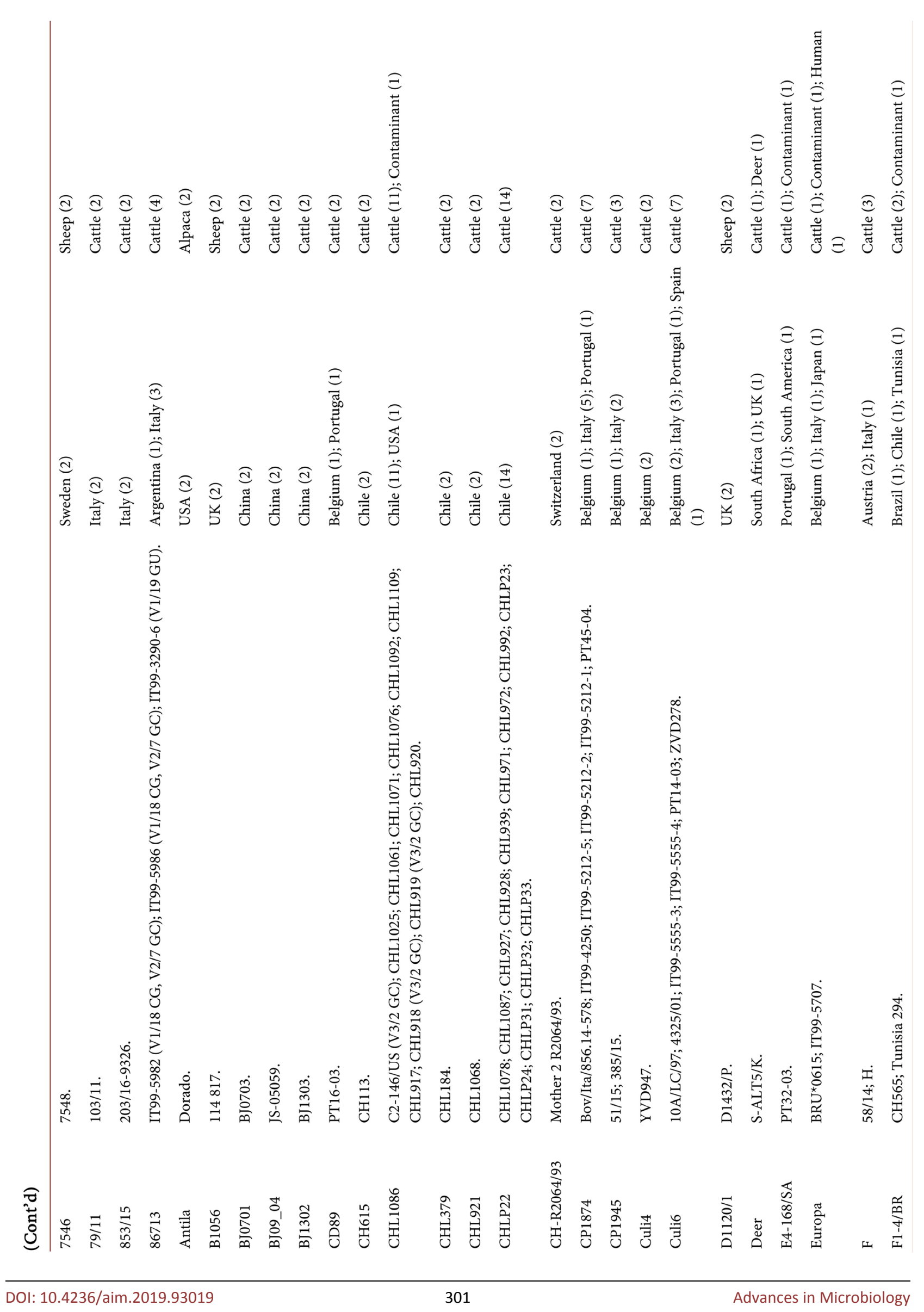




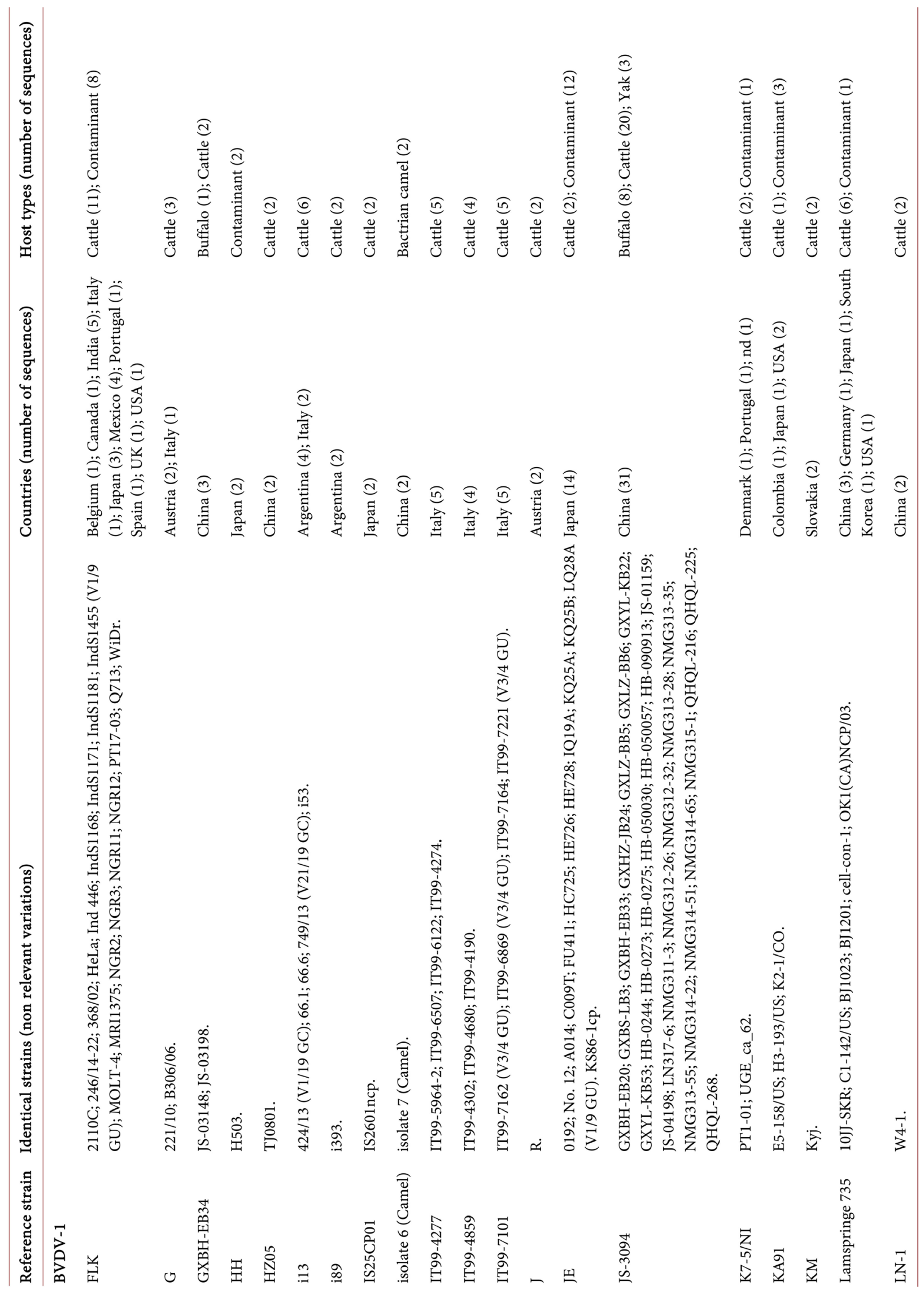




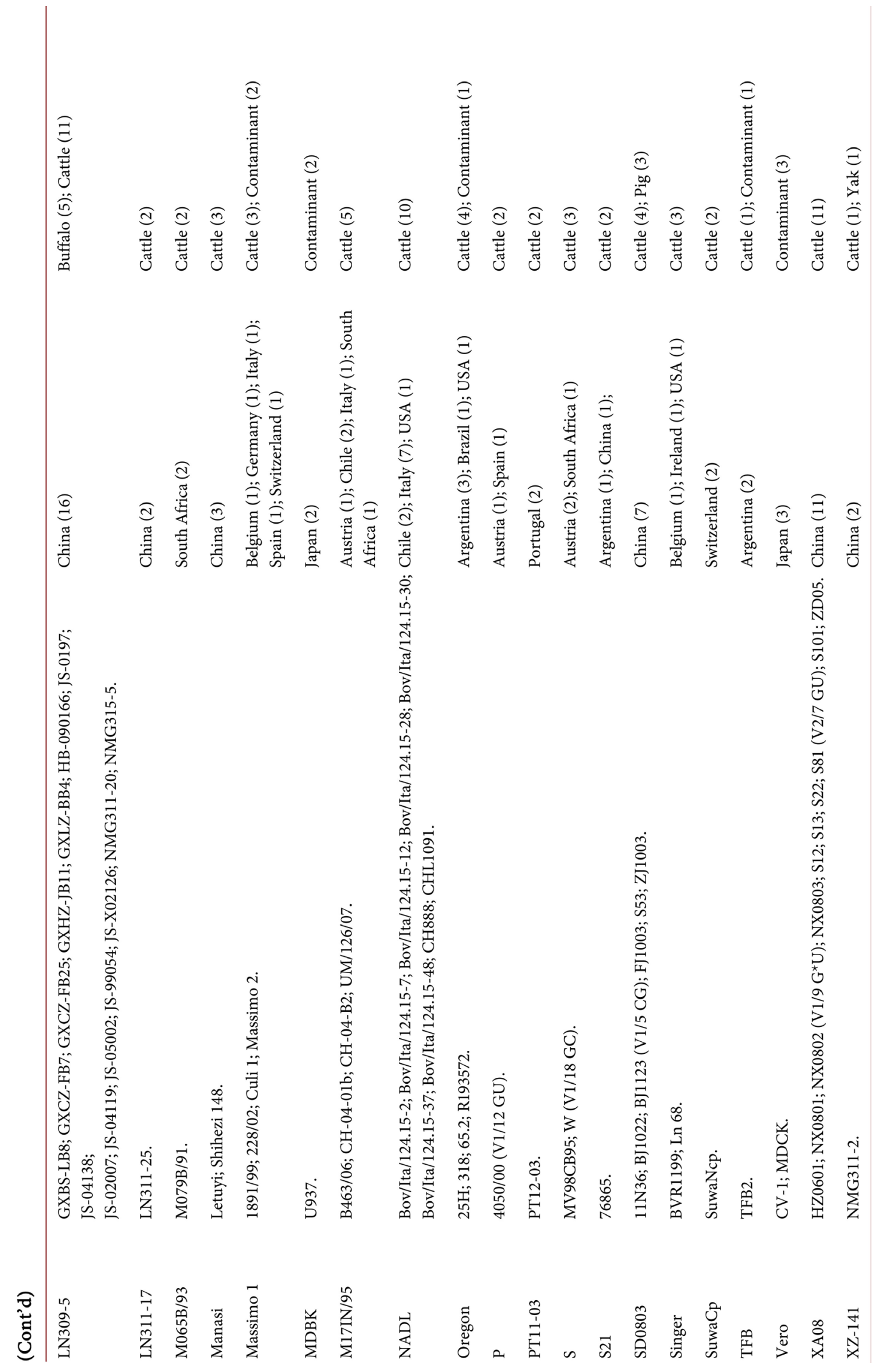




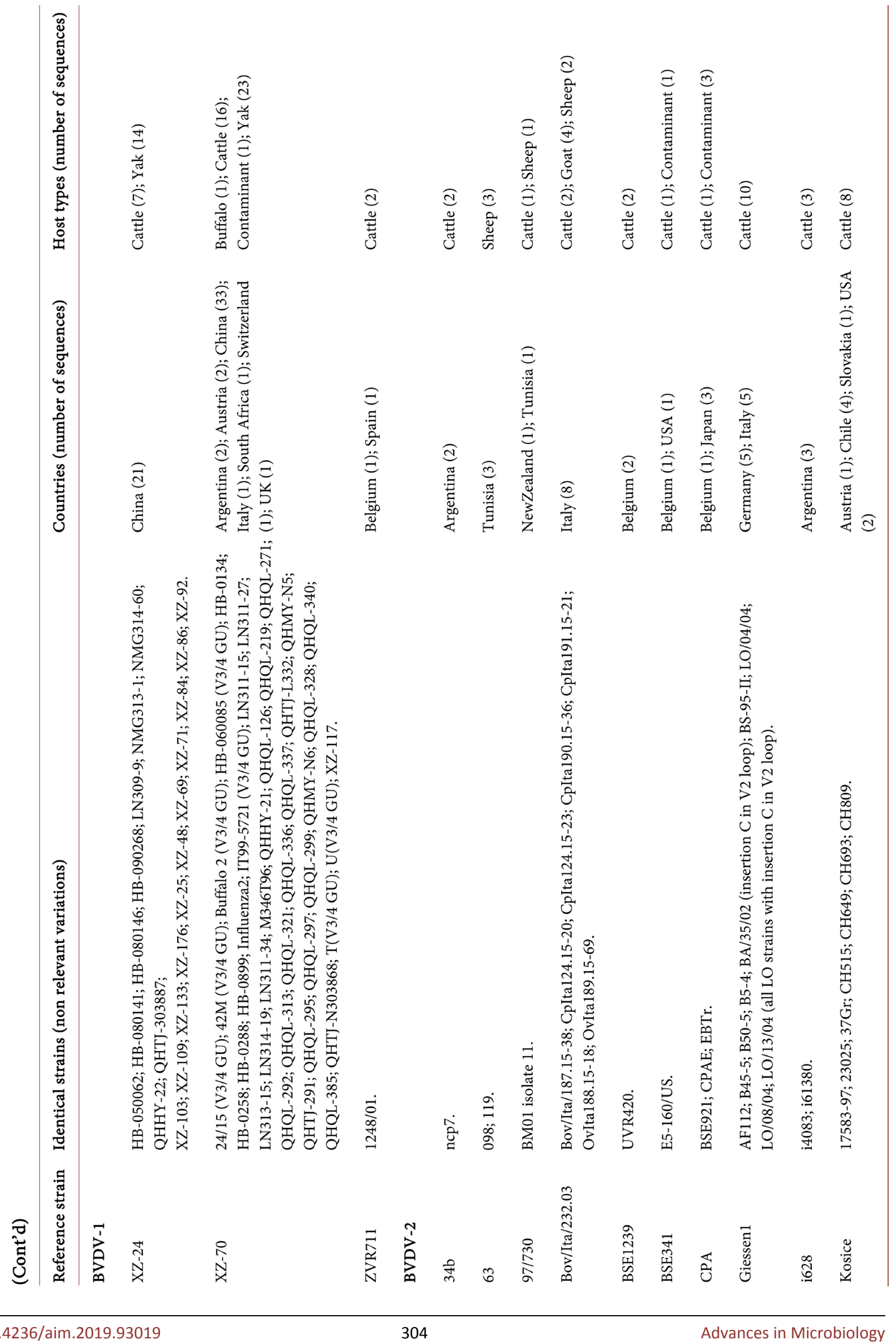



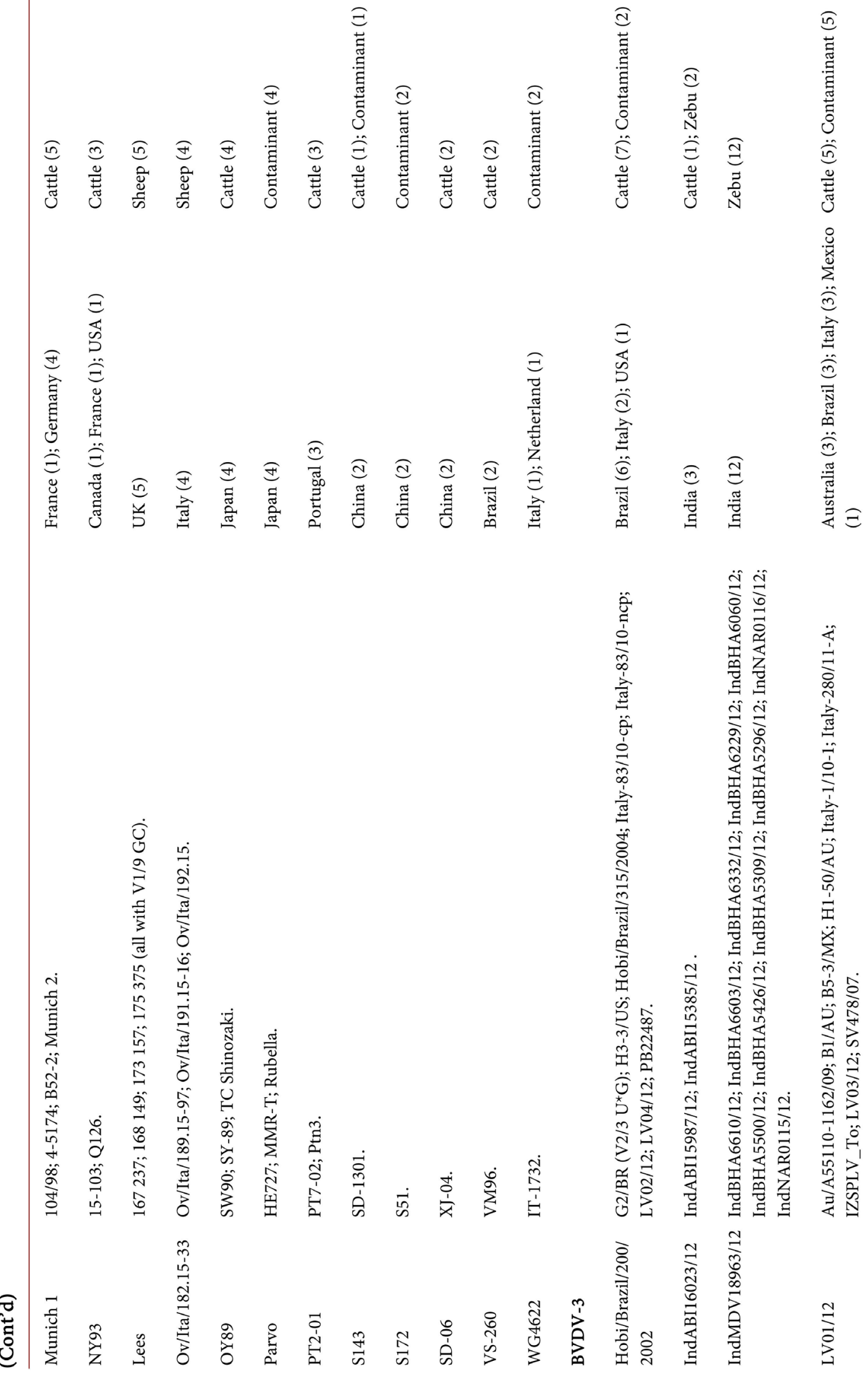


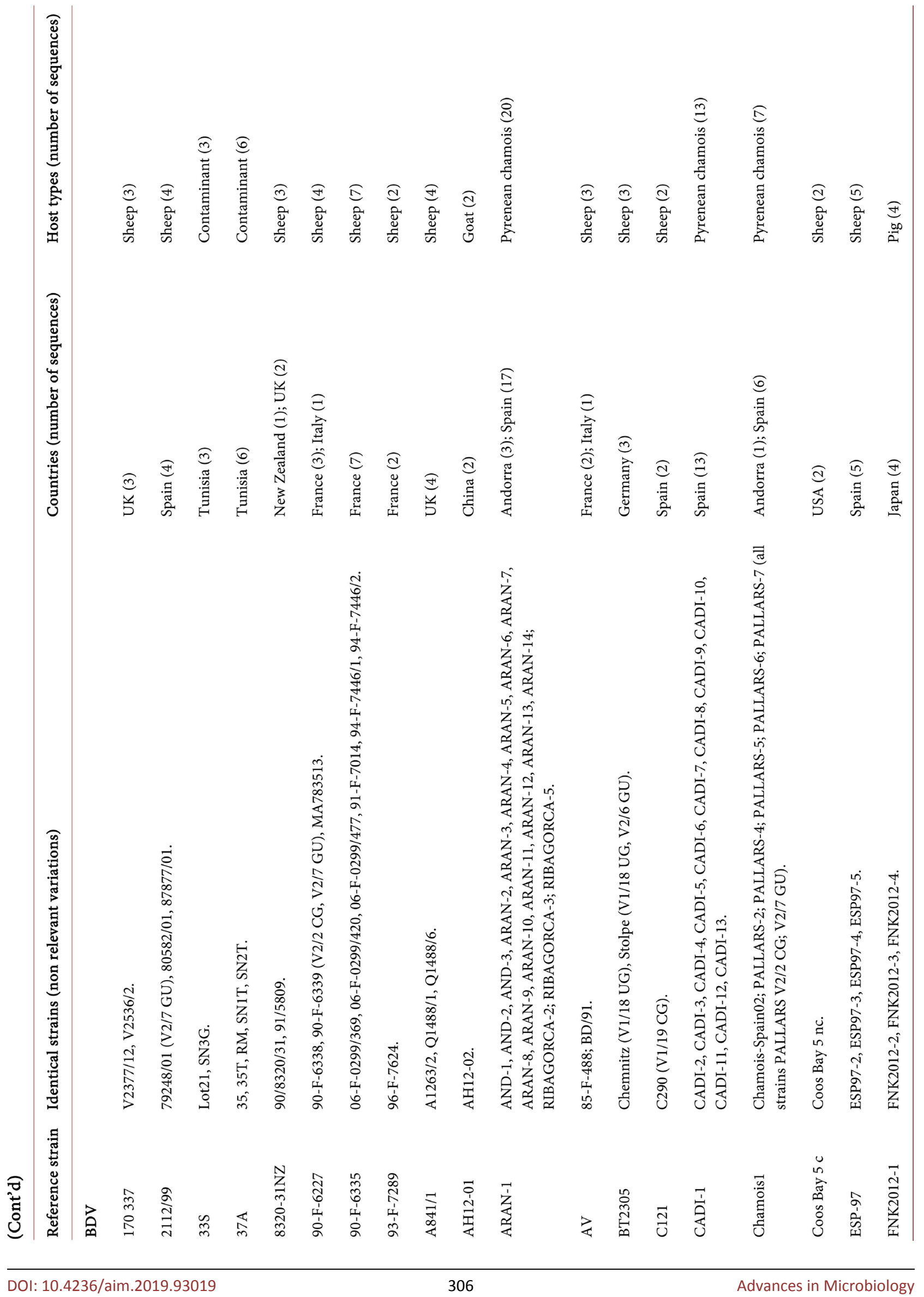




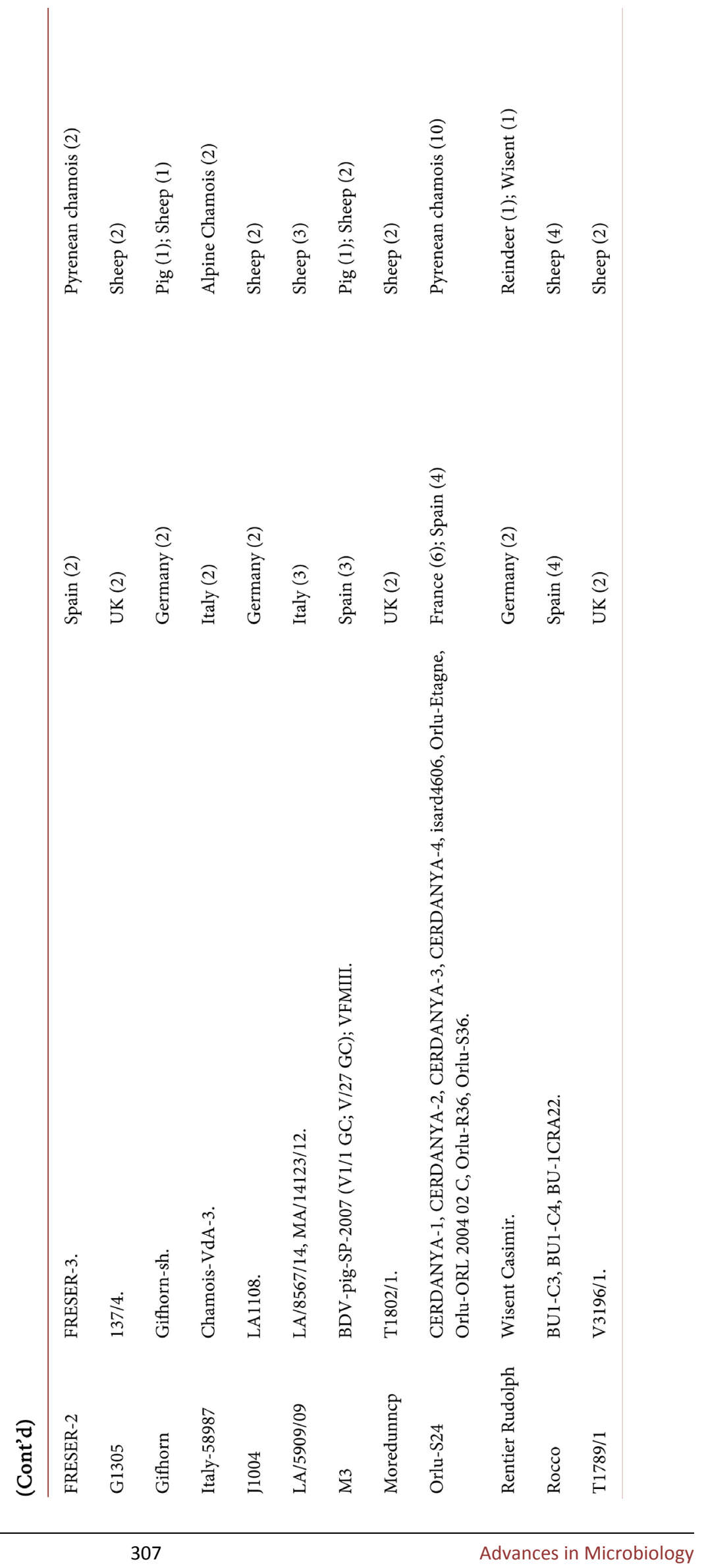




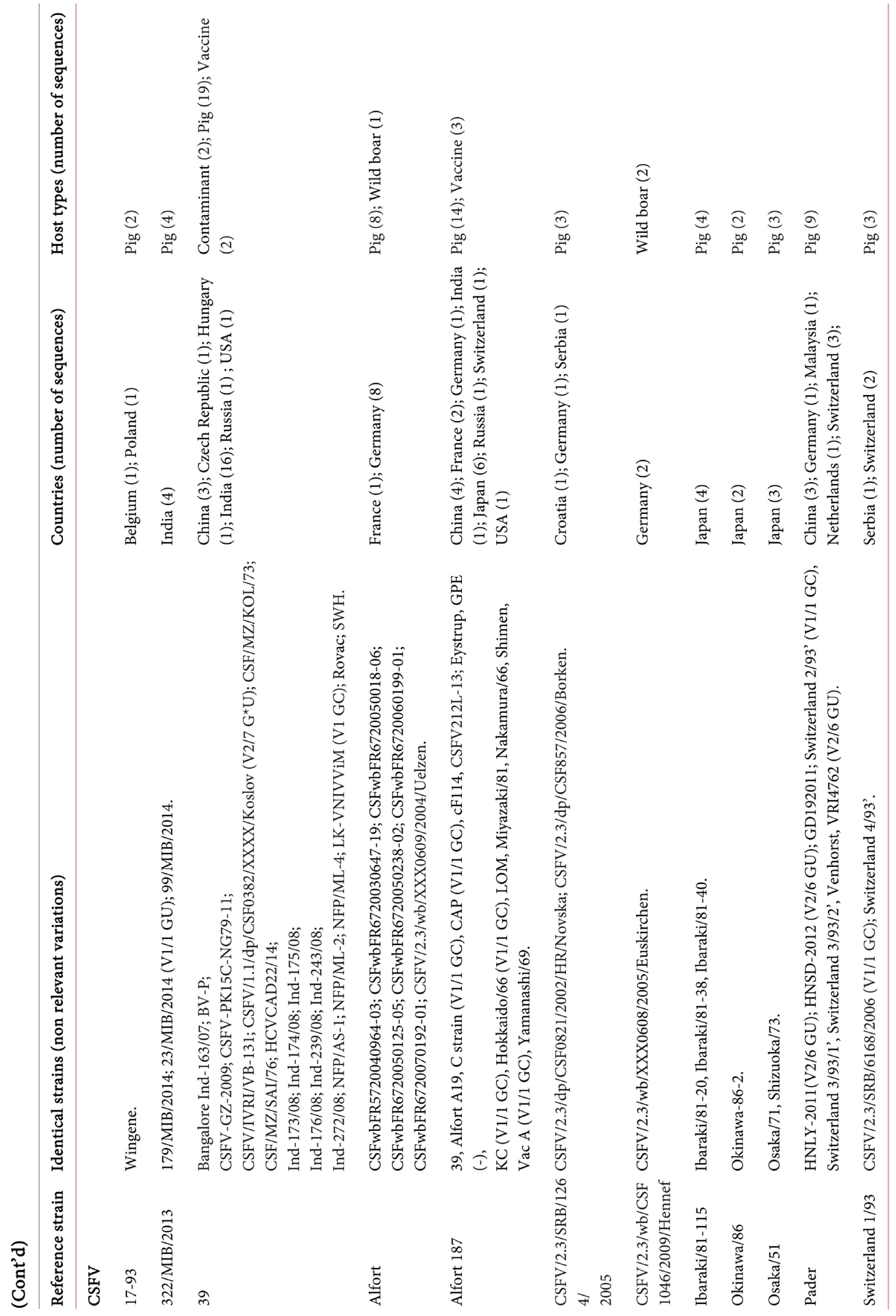




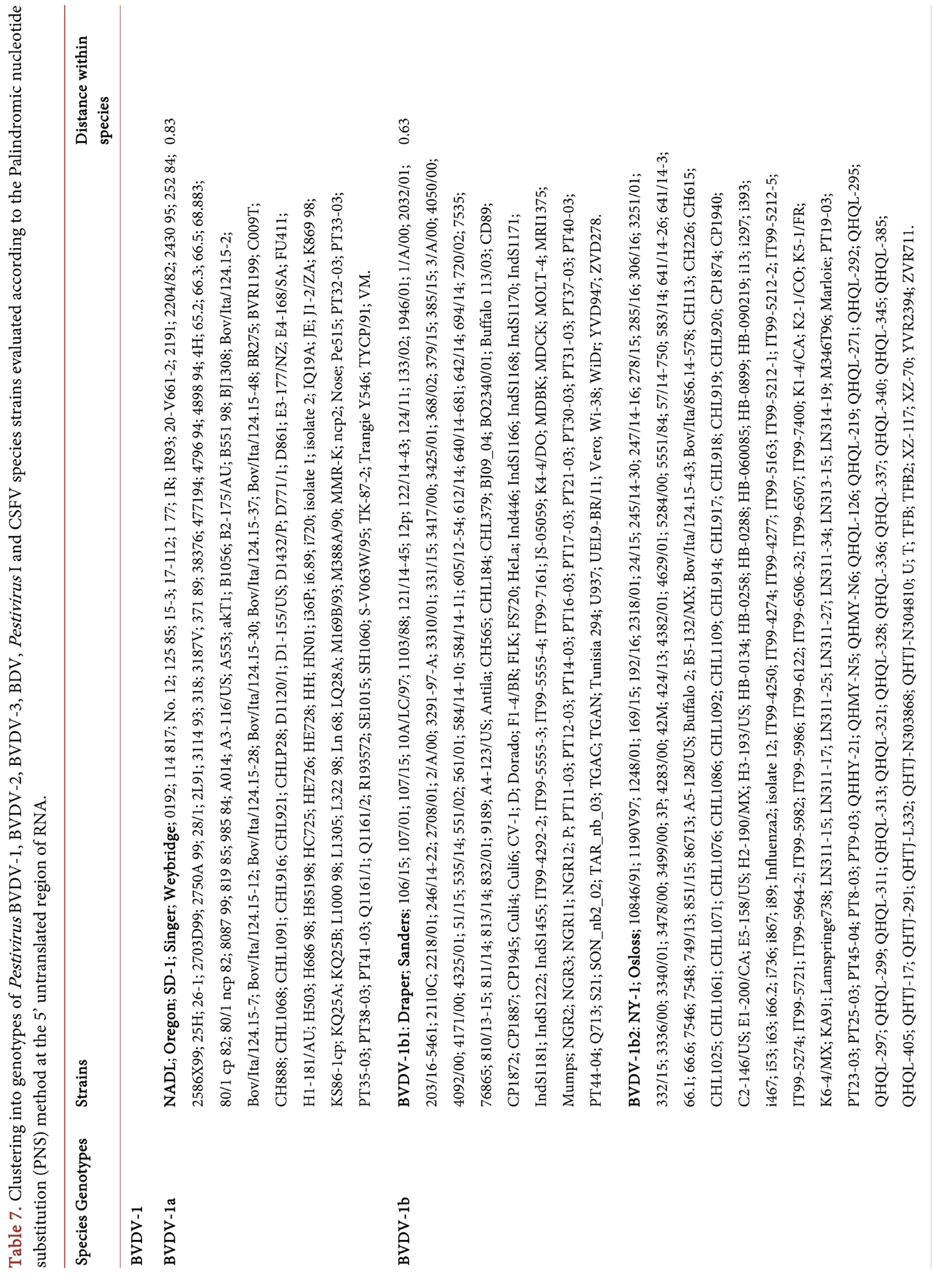


in

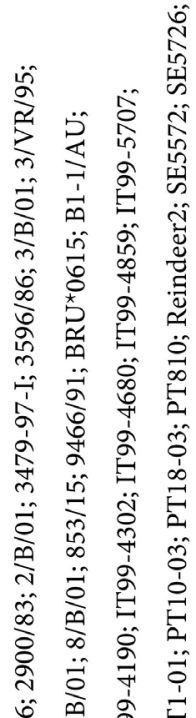

苟

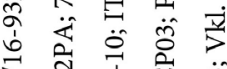

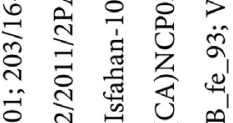

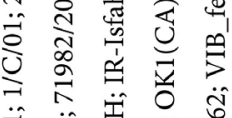

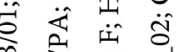

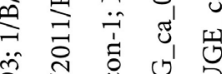

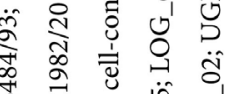

पै

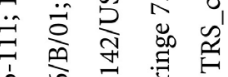

b

空

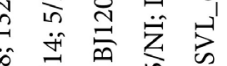

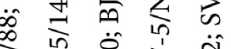

車究

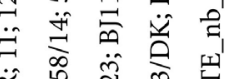

華

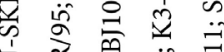

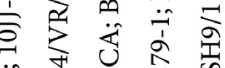

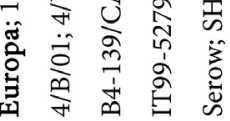

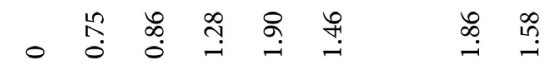

官苓

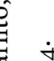

仓े

要

iิ

á

î

$\dot{\infty} \dot{\bar{\sigma}}$

ये है

(1)

है

$\stackrel{8}{\circ}$

$\ddot{N}$ 苛

我

䓎

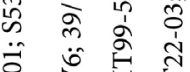

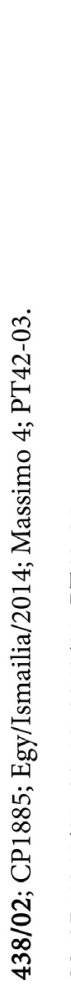

m.

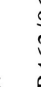

$\stackrel{\circ}{\exists}$

पि

ว่

藏敛

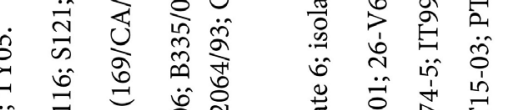

î

ڤิ

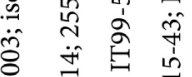

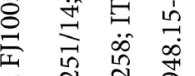

iิ

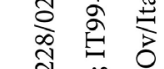

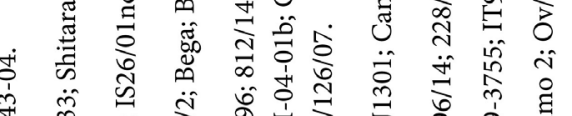

悉

苛

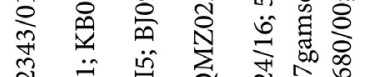

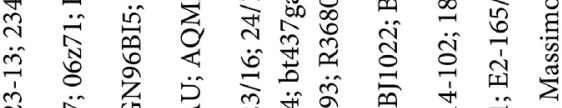

ते

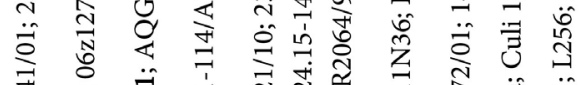

苛

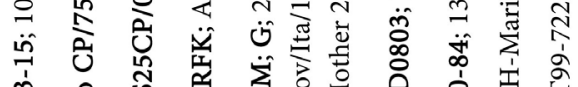

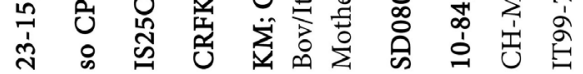

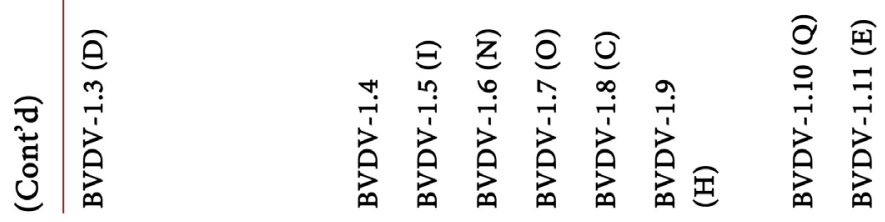




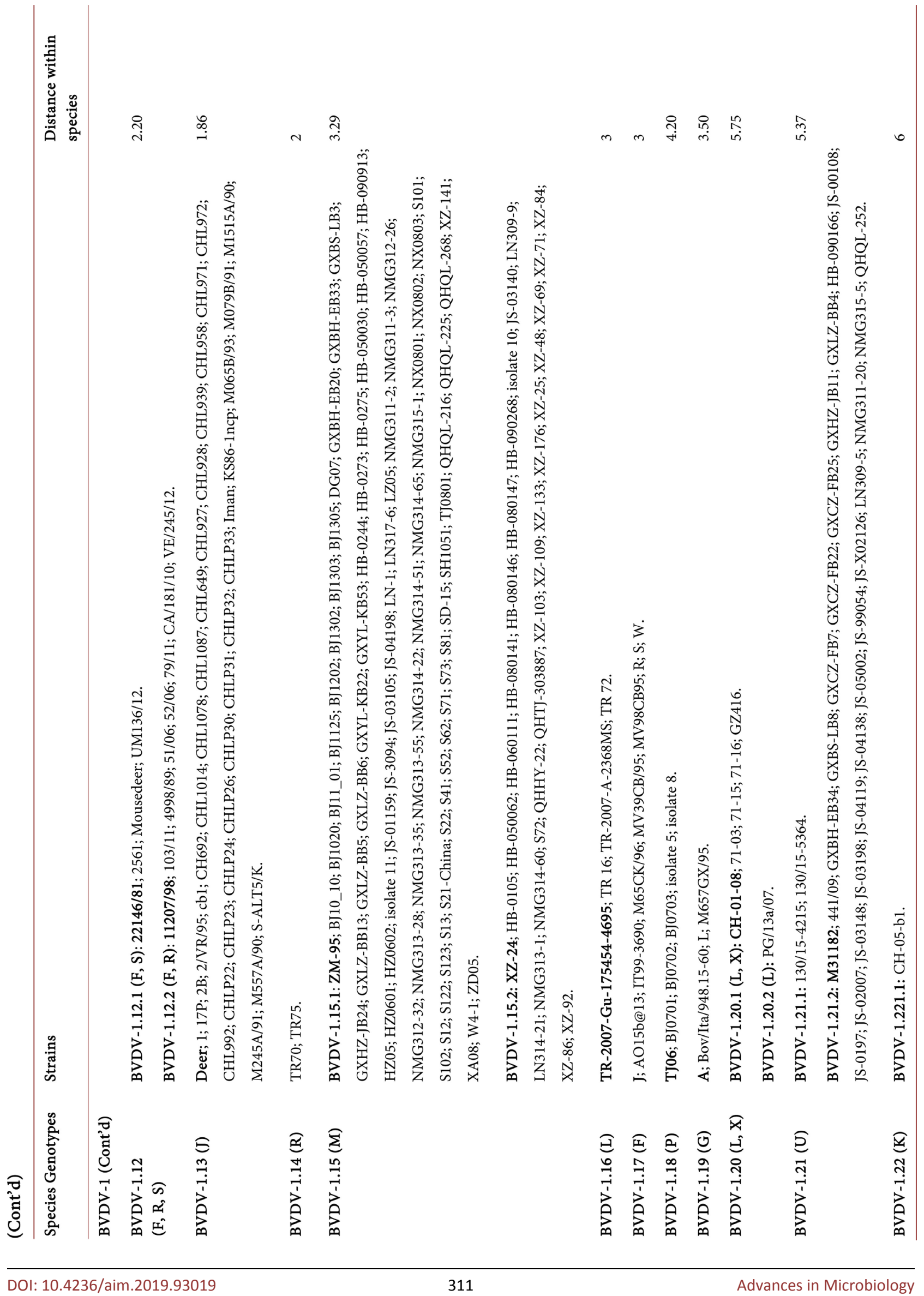




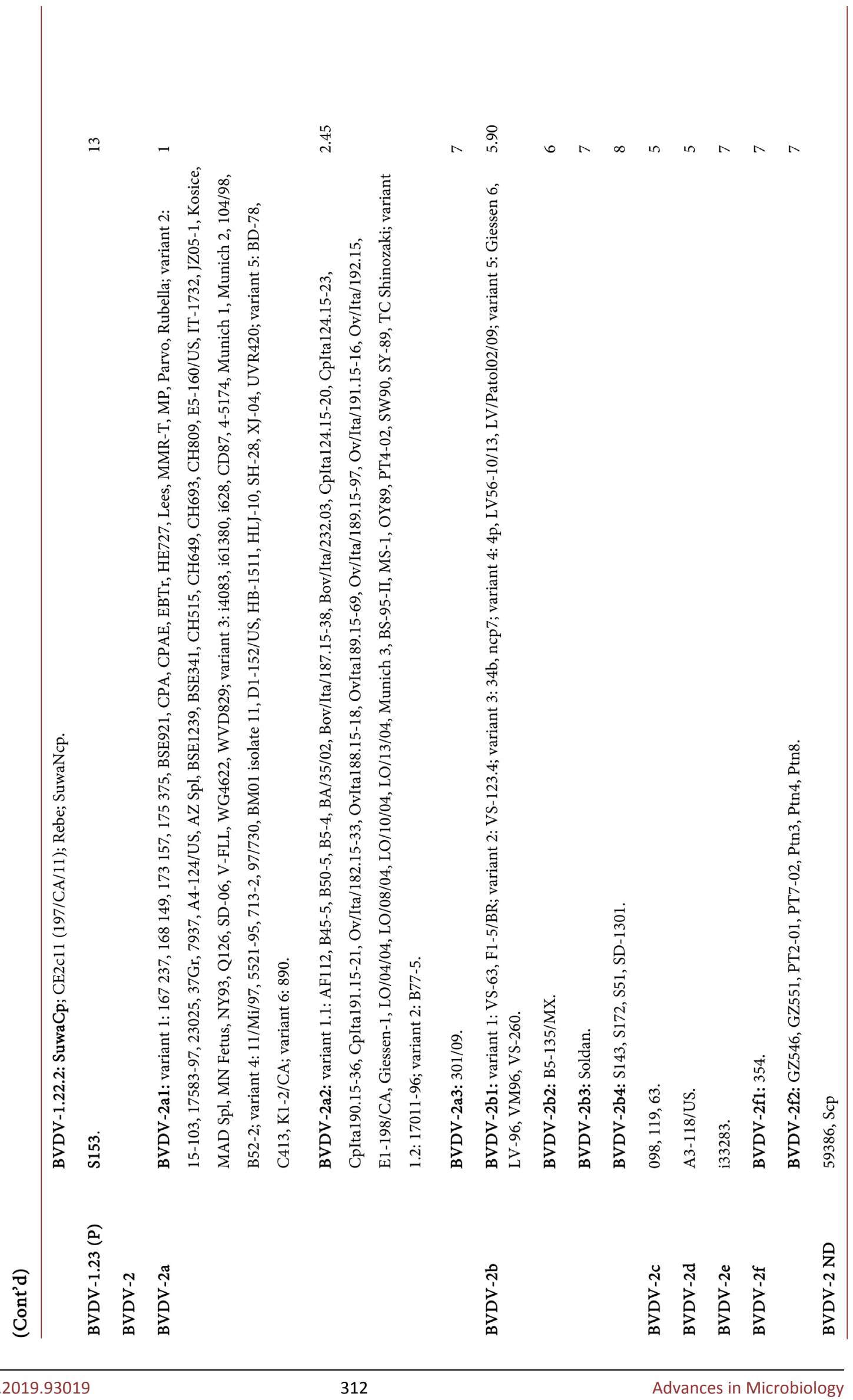




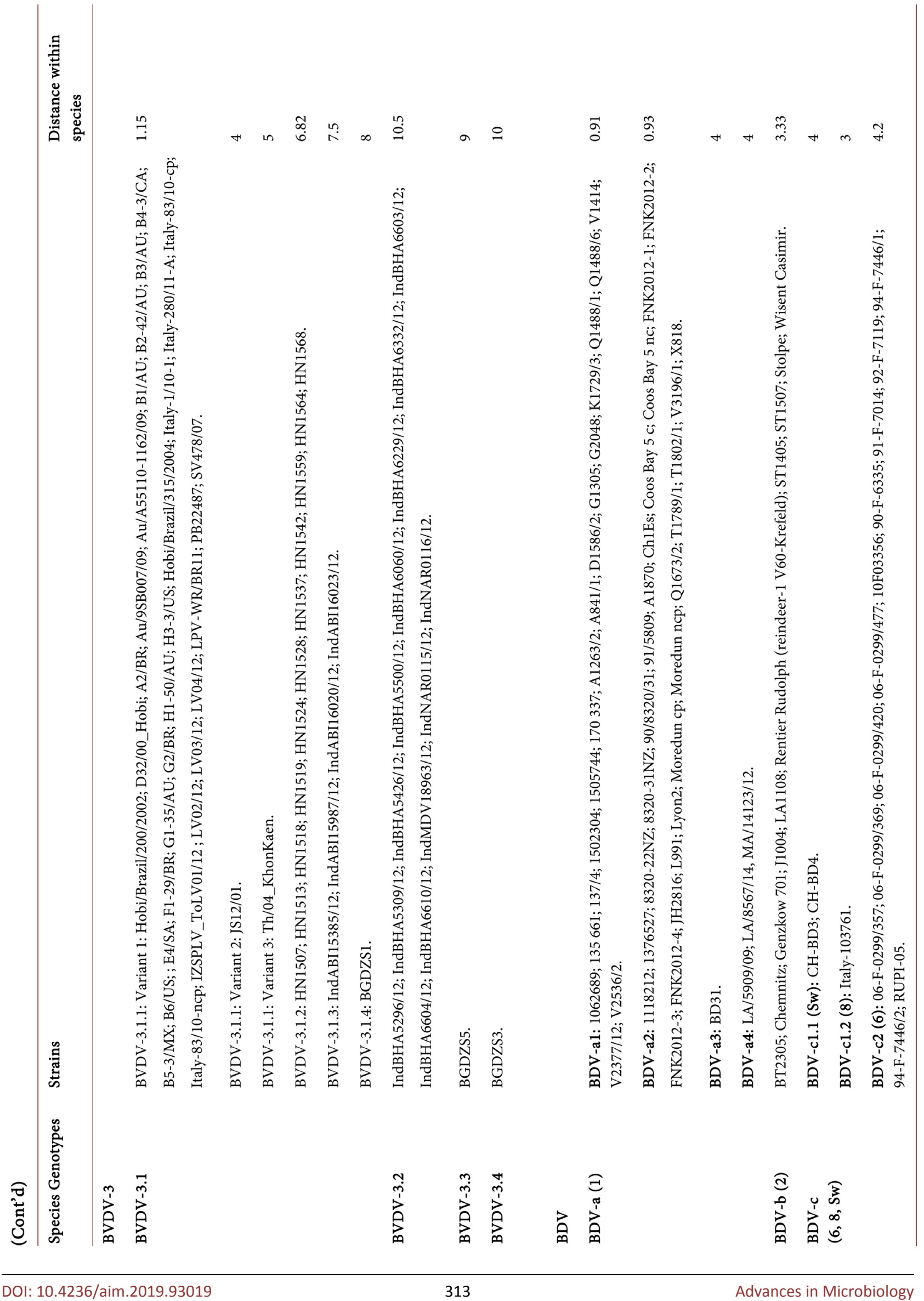




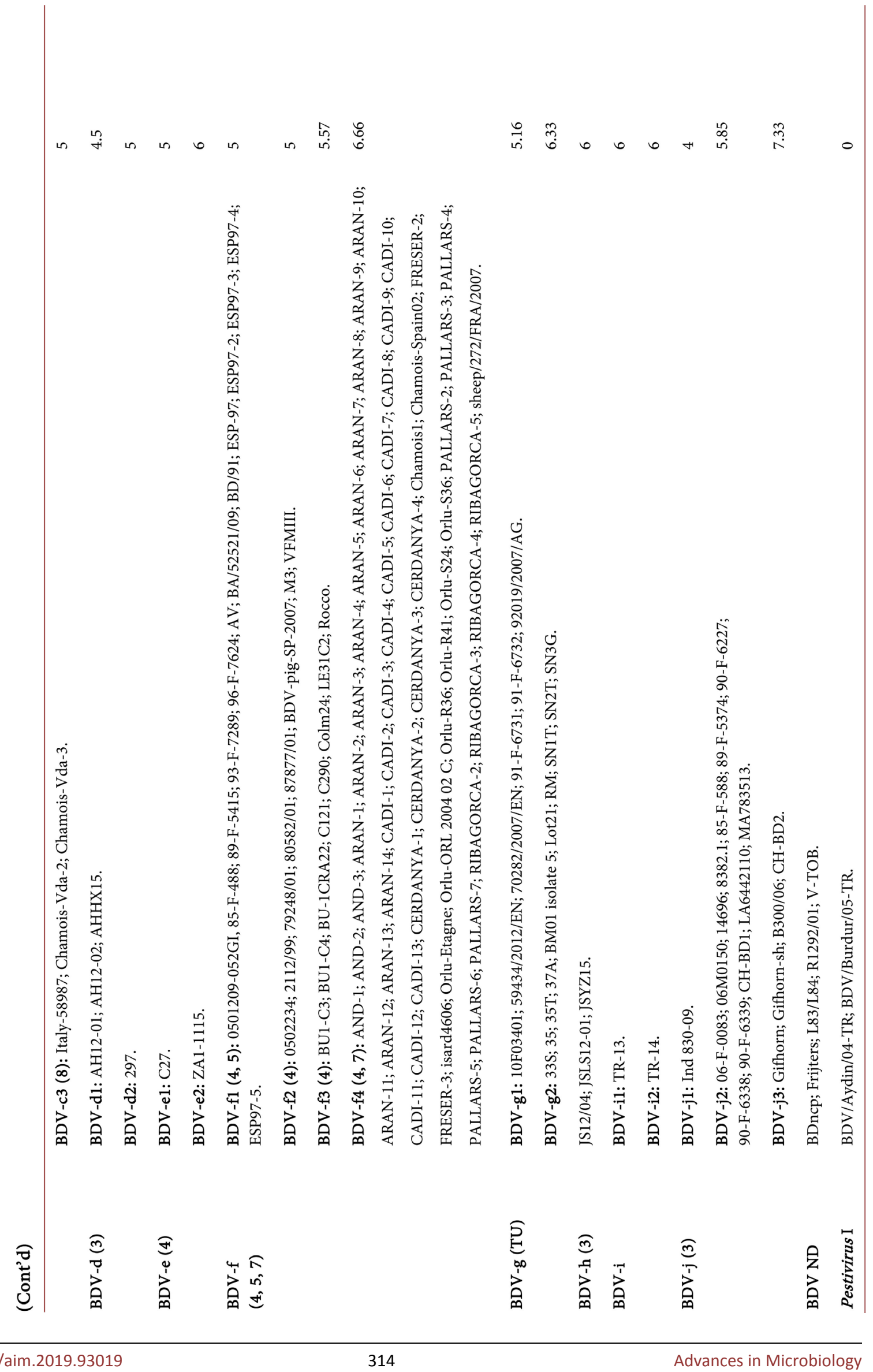




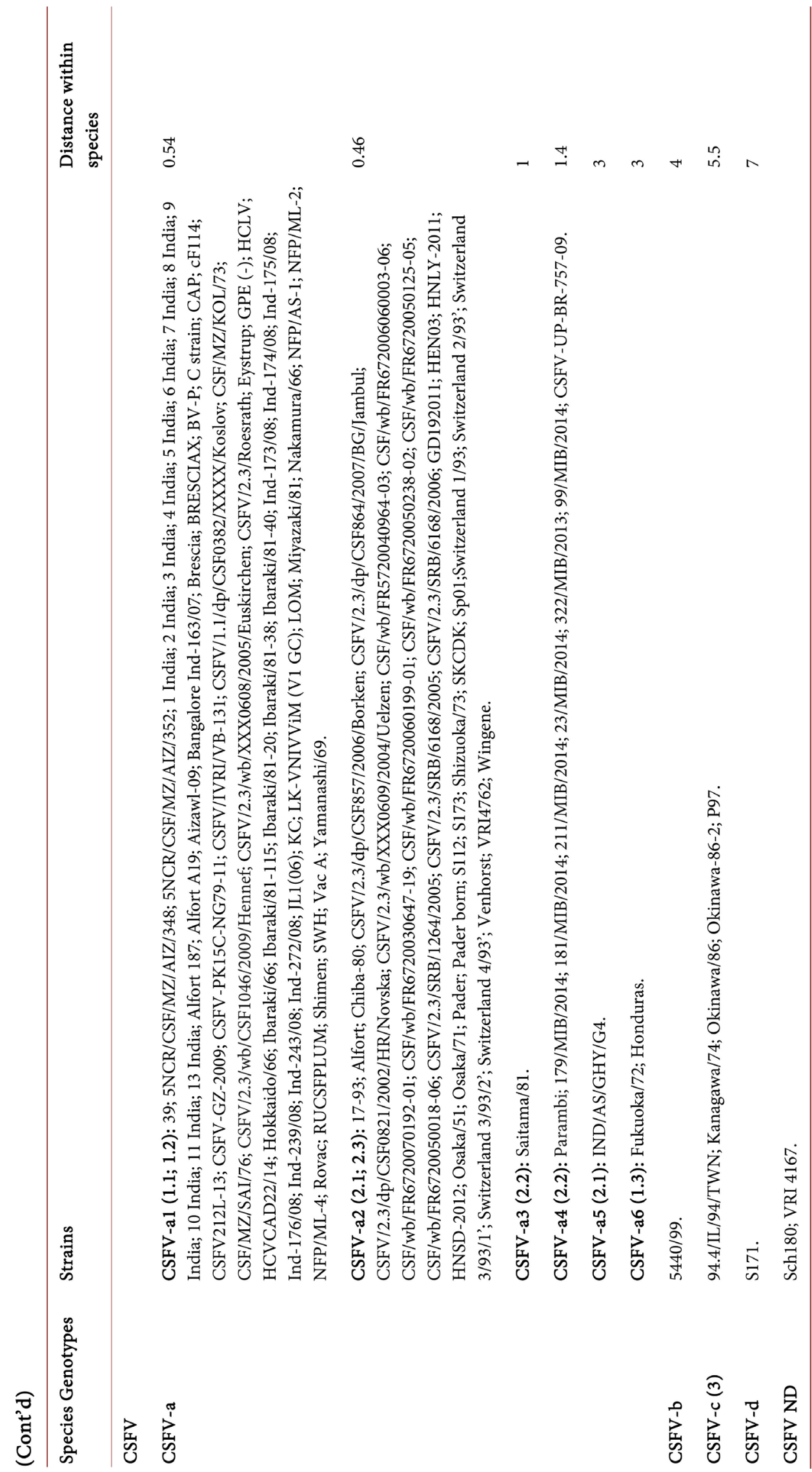


in which was characteristic, and in all the BVDV-2 species strains.

Among BVDV-1 genotypes, only BVDV-1a, 1b, 1.3 and 1.13 were widely distributed. The geographic distribution of genotypes varied considerably, and in certain countries as China, over the half of the 23 genotypes described in the species, 12 could be identified: BVDV-1a, $1 \mathrm{~b} 1$ and $1 \mathrm{~b} 2,1.3,1.6,1.7,1.8,1.10,1.11$, $1.15,1.18,1.21$ and 1.23. BVDV-1a $(\mathrm{n}=4)$ was reported in two bovines [6], two Bactrian camels [31] and one pig [32]. Despite belonging to the same BVDV-1a genotype, strains NH01 (bovine) and SH1060 (pig) were low related, showing $89.2 \%$ homology and a divergence of $11 \mathrm{bp}$ in the IRES. Among BVDV-1b genotype, only two BVDV-b1 have been reported in cattle (Zhang et al., unpublished) [2], while the large majority $(\mathrm{n}=41)$ were BVDV-1b2, 13 in cattle, 27 in yaks [2] and one in a Bactrian camel (Camelus bactrianus) [31]. Four bovine strains (Gao et al., unpublished) [6] belonged to genotype BVDV-1.3. Two other bovine strains (S133 and TY05), reported as M [7] [8] resulted BVDV-1.6. One pig strain, reported as genotype $\mathrm{O}$ by Deng et al. [32] was clustered in the genotype BVDV-1.7, with four other strains all reported as genotype $M$, three from cattle, S43, S121 and BJ0924 [8] and one from camel, isolate 9 [31]. BVDV-1.8 $(\mathrm{n}=5)$ was reported in three bovines [9] and two camels [31]. Genotype BVDV-1.10, reported as $\mathrm{Q}$, resulted circulating in cattle $(\mathrm{n}=6)$, pigs $(\mathrm{n}=5)$ and camels $(\mathrm{n}=$ 3) [3] [4] [6] [8] [31] [32]. Two of the strains from camel, isolate 6 and isolate 7, have been reported as $\mathrm{M}$ by Gao et al. [31]. Only one bovine strain [8] resulted BVDV-1.11. BVDV-1.15.1 (clustered as M) were isolated in 56 bovines, 9 buffaloes, 4 Yaks, [2] [6] [7] [8] [10] 2 pigs [32] and one in a Bactrian camel [31]. BVDV-1.15.2 (28 strains) was reported as M in 13 cattle and 14 yaks [2] [8] and one in a Bactrian camel [31]. Six strains were BVDV-1.18, four cattle isolates reported as $\mathrm{P}$ [7] and two from Bactrian camel, isolate 5 and isolate 8, reported as M [31]. Genotype BVDV-1.21 (U) was reported in 14 bovines, 7 buffaloes and in two yaks (Sun et al., unpublished) [2]. The BVDV-1.23 strain S153 [8] was isolated in cattle. In Japan most of the strains isolated from cattle and contaminant of biological products belonged to BVDV-1 genotypes 1a and 1b1. Genotypes 1.3, 1.6, 1.7, 1.8 and 1.13 were also reported.

Some genetic variants appeared to be restricted in certain areas, as certain genotypes circulating only in Turkey or China, suggesting geographic isolation [7]. Most of BVDV-1 genotypes were related to specific geographic distribution, showing two main populations circulating in Asian and European countries, respectively. Apart few exceptions, a very limited number of strains isolated in Italy belonging to 1.6, 1.8 and 1.21, some genotypes were characteristic from Asia. Genotypes 1.10, 1.15, 1.18 and 1.23 were circulating exclusively in China, and also genotype 1.21 has been reported almost exclusively in China. Other genotypes (1.6, 1.7 and 1.8) appear to be restricted to Asian or Austral Asian countries. U-A pairing in V2/2 was present only among certain Asian genotypes (1.7, 1.10 and 1.15.1). Other genotypes were rare and present only in few countries, BVDV-1.16 bovine strains reported by as genotype L [33], and strains TR70, 


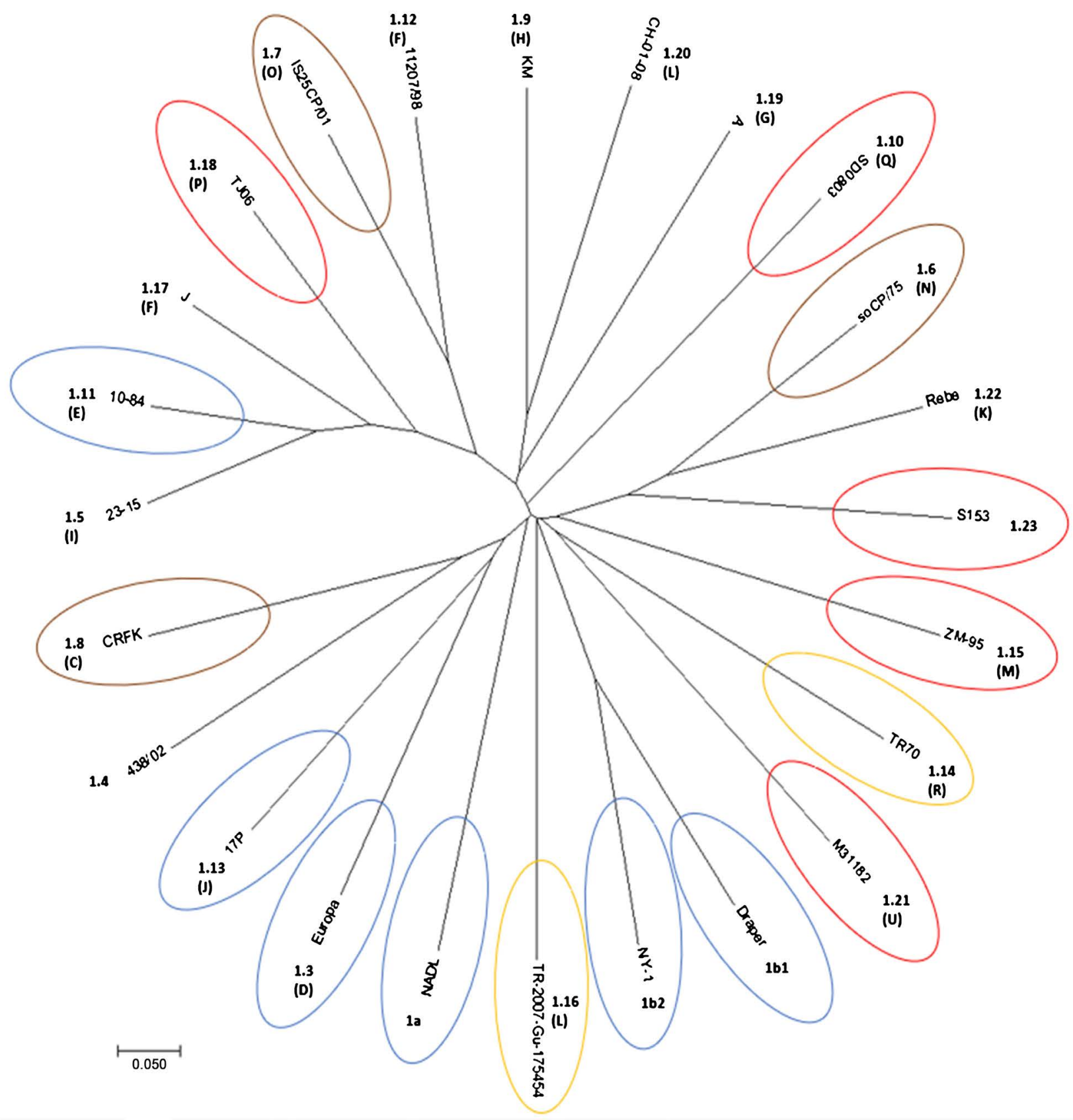

Figure 2. Phylogenetic tree based on the 5'-UTR comparison, suggesting a taxonomic position of the BVDV-1 strains in the genus Pestivirus. Strain NADL [M31182] is the reference for the BVDV-1a genotype, strains Draper [L32880] and NY-1 [L32879] are the references for the BVDV-1b genotype, sub genotypes $1 \mathrm{~b} 1$ and $1 \mathrm{~b} 2$, and strain Europa [AB000898] is the reference for the BVDV-1.3 genotype. Strains 438/02 [AY159540], 23-15 [AF298059], CRFK [D50814], IS25CP/01 [AB359931], so CP/75 [AB042661], KM [AF298068], SD0803 [JN400273], 10-84 [AF298054], 22146/81 [AJ304376], 11207/98 [AJ304390], 17P [AF244954], TR70 [MG670547], ZM-95 [AF526381], TR-2007-Gu-175454-4695 [EU716150], J [AF298067], TJ06 [GU120246], A [AF298064], CH-01-08 [EU180024], M31182 (Yak) [JQ799141], Rebe [AF299317] and S153 [KF006964] are references for the BVDV-1.4 to BVDV-1.23 genotypes. Scale bar indicates 10 nucleotide substitutions per 100 nucleotides. Genotypes present in Asian countries are indicated in circles. Genotypes circulating exclusively in China are indicated in red circles. Genotypes restricted to Turkey are indicated in orange circles. Those restricted to Far East Asia and Austral Asia (Japan, South Korea, China and Australia) are indicated in brown circles. A very limited number of strains isolated in Italy belonging to 1.6, 1.8 and 1.21 represented exceptions. Nomenclature of identified genotypes is based on divergence in the genus. Clustering according to primary structure analysis by depositors is indicated under parenthesis. 


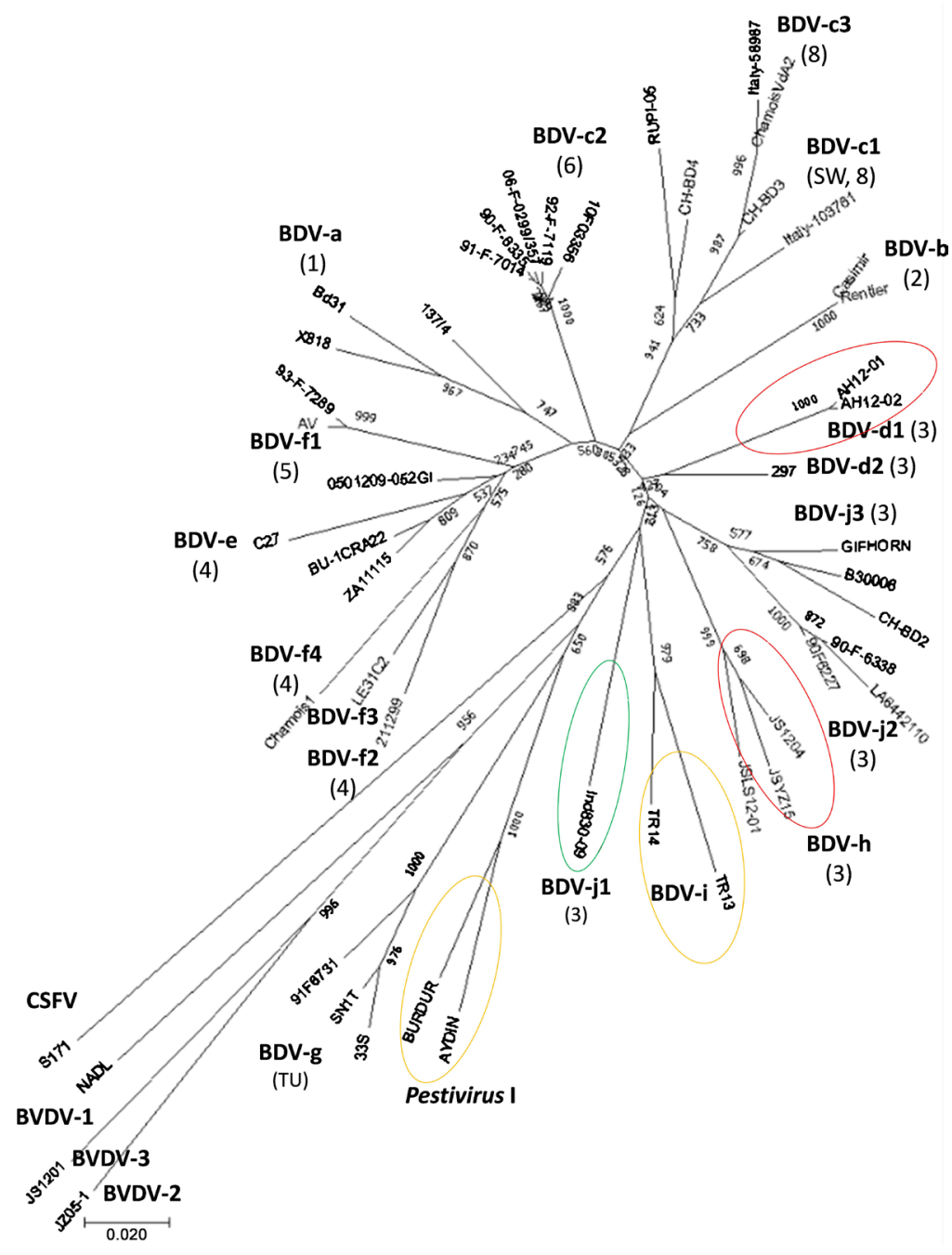

Figure 3. Phylogenetic tree based on the 5'-UTR comparison, suggesting a taxonomic position of the BDV strains in the genus Pestivirus. Strains 137/4 [U65052], X818 [AF037405], Bd31 [U70263], Casimir [AB122085] and Rentier [AB122086] are references for the genotypes BDV-a sub genotypes 1, 2 and 3 and genotype BDV-b. Strains CH-BD3 [JQ994199], CH-BD4 [JQ994200], Italy-58987 [KX573913], Italy-103761 [KT072634] and ChamoisVdA2 [MG725337] are references for the genotype BDV-c sub genotypes 1 and 3 and the strains 92-F-7119 [EF693994], 92-F-7014 [EF693993], 90-F-6335 [EF693990], 06-F-0299/357 [EF694000], 10F03356 [KC859384] and RUPI-05 [KC859383] are references for the genotype BDV-c sub genotype 2. The Chinese strains AH12-01 [JQ946320] and AH12-02 [JX437132] are reference for the genotype BDV-d sub genotype 1, and strain 297 [KC484999] is reference for the BDV-d sub genotype 2. Strains C27 [DQ275623] and ZA11115 [DQ361070] are references for the BDV-e sub genotypes 1 and 2. Strains AV [EF693984], 0501209-052GI [DQ679902] and 93-F-7289 [EF693995] are references for the BDV-f sub genotype 1. Strains 2112/99 [AY159513], BU-1CRA22 [DQ275622], LE31C2 [DQ361072] and Chamois1 [AY738080] are references for the BDV-f sub genotypes 2, 3 and 4. The strain 91-F-6731 [EF988632], 33S [AF462002] and SN1T [AF461997] are reference for the genotype BDV-g (Tunisian type). The Chinese strains JS12/04 [KC537789], JSLS12-01 [KC963426] and JSYZ15 [KT327869] represent the genotype BDV-h. The Indian strain Ind830-09 [KT934377] is reference for the genotype BDV-j sub genotype 1, the strains 90-F-6338 [EF693991], 90F6227 [EF693989], LA/64421/10 [LM999989], Gifhorn [EU636997], B30006 [EU224227] and CH-BD2 [JQ994198] are reference for the genotype BDV-j sub genotypes 2 and 3. The Turkish strains TR13 [JF489888] and TR14 [JF489889] are references for the genotype BDV-i. The bovine strains NADL [M31182], the Chinese bovine strains JZ05-1 [GQ888686], JS1201 [JX469119] and S171 [KF006974] represents the BVDV-1, BVDV-2, BVDV-3 and CSFV species. The ovine Turkish strains Aydin [AM418427] and Burdur [AM418428] represents the Pestivirus I species. Scale bar indicates 10 nucleotide substitutions per 100 nucleotides. Genotypes present only in Asian countries are indicated in circles (red: China; green: India; orange: Turkey). Nomenclature of identified genotypes is based on divergence in the genus. Clustering according to primary structure analysis by depositors is indicated under parenthesis. 


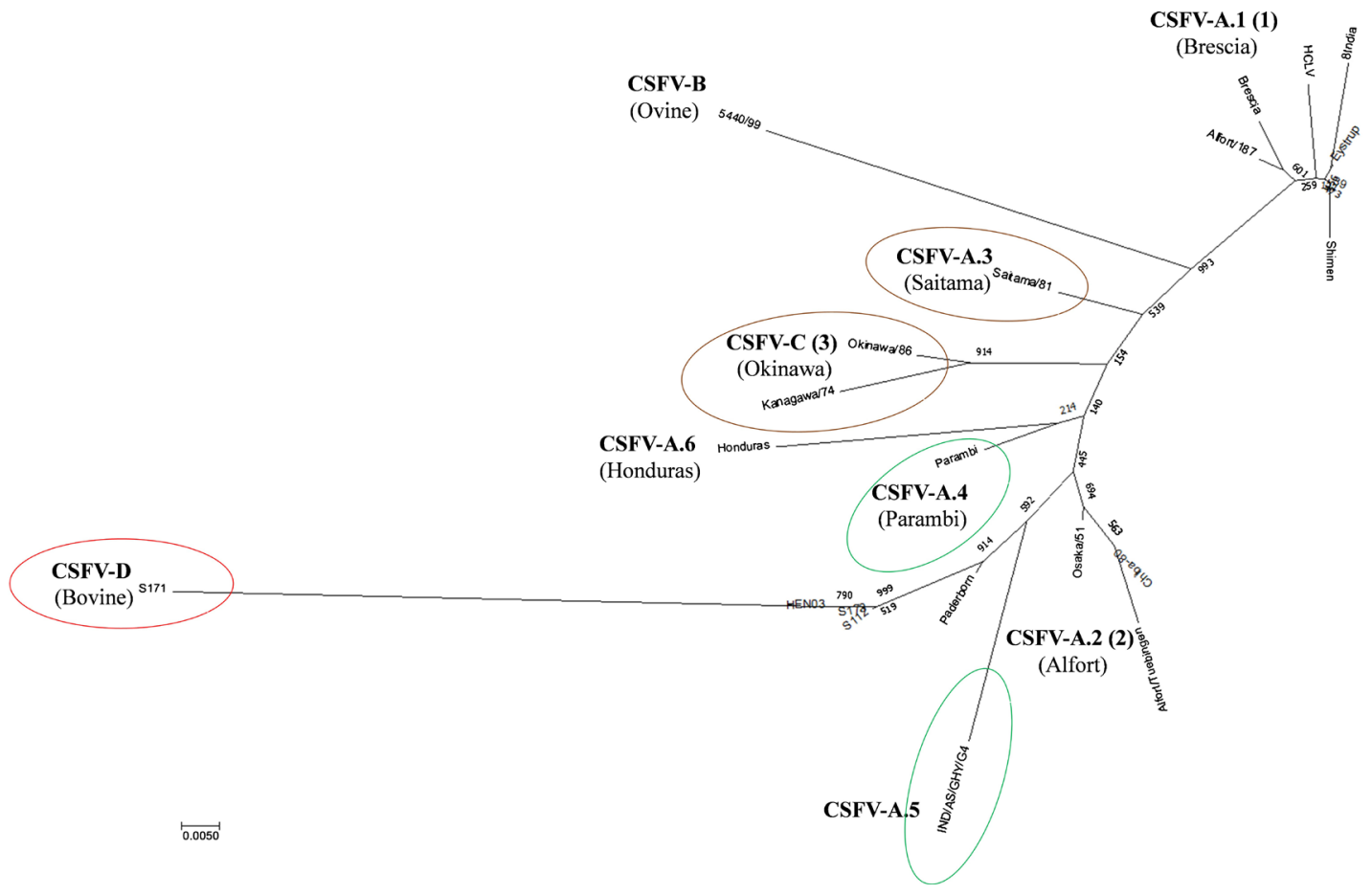

Figure 4. Phylogenetic tree based on the 5'-UTR comparison, suggesting a taxonomic position of the CSFV strains in the genus Pestivirus. Strains Brescia [M31768], 39 [AF407339], Eystrup [AF326963], Alfort/187 [X87939], HCLV [AF091507], Shimen [AF092448] and the bovine strain 8 India [MG813566] are references for the genotype CSFV-a variant 1. Strains Alfort/Tuebingen [J04358], Chiba-80 [AB019659], Osaka/51 [AB019174], Paderborn [AY072924] and the Chinese bovine strains S173 [KF006975], HEN03 [KC176778] and S112 [MK118725] are references for the genotype CSFV-a variant 2. Strain Saitama/81 [AB019178] is reference for the CSFV-a variant 3, and strains Parambi [KT239105] and Honduras [L42426] are reference for the CSFV-a variants 4 and 6. The Spanish ovine strain 5440/99 [AY159514] is reference for the genotype CSFV-b. The Japanese strains Kanagawa/74 [AB019166] and Okinawa/86 [AB019172] are references for the genotype CSFV-c. The Chinese bovine strain S171 [KF006974] represents the genotype CSFV-d. Scale bar indicates 10 nucleotide substitutions per 100 nucleotides. Genotypes present only in Asian countries are indicated in circles (red: China; green: India; brown: Japan and Taiwan). Nomenclature of identified genotypes is based on divergence in the genus. Clustering according to primary structure analysis by depositors is indicated under parenthesis.

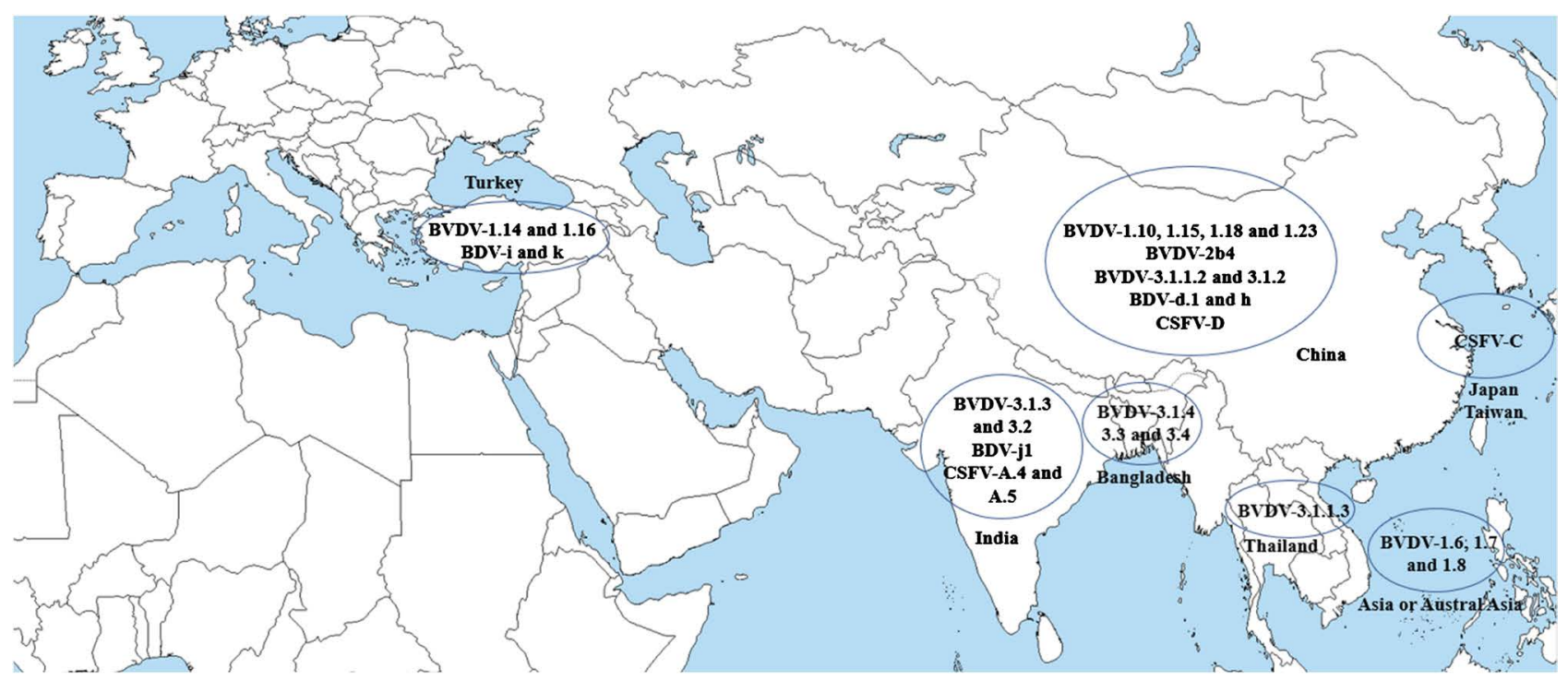

Figure 5. Schematic representation of geographic distribution of Asian Pestivirus species genotypes circulating in restricted areas. 
TR73 and TR75 belonging to the BVDV-1.14, reported by Yesilbag et al. [34] as genotype $\mathrm{R}$, were restricted to Turkey.

In the BVDV-2 species, while BVDV-2a showed cosmopolitan diffusion and $2 \mathrm{~b}$ and other related genotypes were more frequently distributed in South America, only one group appeared specific to Asia. The genotype b variant 4 (BVDV-2b4) included only Chinese isolates: the bovine strain SD-1301 [5] and the contaminants S143, S172 and S51 [8]. BVDV-3 species showed low heterogeneity with four different genotypes. Out of four, three (BVDV-3.2, BVDV-3.3 and BVDV-3.4) were specific to zebu and bovine isolates from India and Bangladesh, respectively [12] [13]. Strains IndMDV18963/12 and IndBHA6604/12, representative for BVDV-3.2 genotype, were divergent in the species, scoring divergence $96.66 \%$ when compared with other BVDV-3 strains. Similarly, with divergence values of $82.14 \%$ for BGD/ZS5 and $100 \%$ for BGD/ZS3, respectively, bovine strains from Bangladesh were divergent from genotypes 1 and 2, constituting a separate genotype in the BVDV-3 species. In the BVDV-3 genotype 1 , subgenotype 1 variants 2 (contaminant strain JS12/01) [35] and 3 (bovine strain Th/04_KhonKaen) [36] were reported from China and Thailand. BVDV-3 genotype 1 subgenotype 2 was specific to small ruminants from China (Shi et al., unpublished), with divergence $27.27 \%$, divergence mean value 6.82 from other BVDV-3.1 genotype strains. BVDV-3 genotype 1 subgenotypes 3 and 4 were specific to zebu and bovine isolates from India and Bangladesh, respectively [12] [13]. Strains IndABI16023/12 and IndABI16020/12, representative for BVDV-3.1.3, showed a divergence of $23.33 \%$ from strains of other genotype variants. BVDV-3.1.4 strain BGDZS1 showed a divergence of $40 \%$.

BDV species sequence characteristics of Chinese and Turkish strains were highly divergent from other genogroups, indicating geographic segregation. Chinese strains AH12-01, AH12-02 and AHHX15, reported as genotype BDV-3 (Ghiforn type-PNS BDV-j) [37], have been clustered as genotype BDV-d, sub genotype d1. These strains showed high homology with strain 297, also reported as BDV-3 [38], clustered in the same genotype, but as separate sub genotype BDV-d2. Similarly, other Chinese strains JS12/04, JSLS12-01 and JSYZ15, reported as genotype BDV-3 [37] have been clustered as genotype BDV-h. Both genotypes resulted partially related with $\mathrm{BDV}-\mathrm{j}$. However, qualitative non correspondence at root LVP level (BDV-d GC/GU/UA-AA-AG; BDV-h GC/GU-AU/UA-AA-AU; $B D V-j$ GC/AU/UA-UG-GC-UC) (exception AA in $V 3 / 8$ of strain BDV-j2 $\mathrm{CH}-\mathrm{BD} 1$, discriminated from $\mathrm{BDV}-\mathrm{h}$ with additional identification marker V1/3: BDV-j2 G-C; BDV-h A-U) and quantitative evaluation of divergence values justified separate clustering. Strains of sub genotype BDV-d1 were related only with BDV-j1 (India type) (no divergence values exceeding genotype limit value 9; divergence mean value 8.5 ) and divergent from sub genotypes BDV-j2 (divergence 92.85\%, divergence mean value 10.78) and BDV-j3 (divergence $100 \%$, divergence mean value 11.83). Strain 297 (sub genotype BDV-d2) resulted divergent from BDV-j3 (Ghiforn type) (divergence 100\%, divergence mean value 
11) and related with BDV-j1 and BDV-j2 (divergence mean values 8 and 8.14, respectively), but showing lowest divergence value mean of 6.5 with BDV-d1 strains. BDV-h resulted related only with BDV-j1 (homology 33.33\%; divergence mean value 8.67) and divergent from other BDV-j sub genotypes (BDV-j2: divergence $61.9 \%$, divergence mean value 9.67 ; $\mathrm{BDV}$-j3: divergence $100 \%$, divergence mean value 12.33).

Turkish strains TR-13 and TR-14, reported as distinct group in the BDV species [39], have been clustered as genotype BDV-i. Due to the divergence value of 9 , obtained comparing their respective sequences, related to nucleotide variations particularly in the V1 stem, but maintaining clear base pairing homology in the rest of the secondary structure, they have been considered as two separate sub genotypes. BDV-i1 strain TR-13 showed bulges in V1/8 C C and V1/9 G A and BDV-i2 strain TR-14 showed V1/4 G A bulge, V2/12 insertion U and V1/8 A $G$ bulge as characteristic bp in the sequence. Comparison with the other BDV sequences, applying species determination limit value 13 , resulted related to the majority of BDV genotypes, showing a divergence percentage of 36.08 , with divergence value range from 7 to 19 (mean 12.91). High divergence values have been observed with genotype BDV-f (including Aveyron and Pyrenean Chamois types) (values up to 17 - 19), BDV-g (Tunisian type), sub genotype 2 (values 15 16). High divergence values (16-19) have been obtained also with Pestivirus I (Turkish type Aydin-Burdur). However, strains TR-13 and TR-14 shared all BDV species markers. Comparison with the other BDV sequences, applying genotype determination limit value 9, resulted divergent from all other BDV genotypes, despite the partial relation scored with some members of genotype BDV-a, sub genotype 2, BDV-c, sub genotype 1 , and BVD-j, which, however, did not correspond to relatedness when considering the entire group to determine overall homology (out of 192 comparisons, only 19 values were 7 - 9 and 13 corresponded to the limit value). Also BDV-j1 included the Indian ovine strain Ind 830-09 [40].

In the CSFV species, secondary structure analysis revealed corresponding main genomic groups, defined by phylogenetic analysis, dividing CSFVs into three major lineages (Brescia, Alfort and Kanagawa/Okinawa types) and their sublineages [41]. Three genetic clusters referred specifically to Asian countries. The CSFV genotype a variant 4 (type Parambi) included only pig and wild boar strains from India (Ravishankar et al., Tomar et al., Chandramohan et al., unpublished) [42]. CSFV pig strains clustered into genotype C (type Okinawa) were reported only from Japan and Taiwan (Liu, unpublished) [41] [43] [44]. The Chinese strain S171 [8], isolated from bovine serum, was clustered as CSFV-d. The strain S171 showed affinity with genotype CSFV-a2, but the difference was marked at the level of the V3 locus nucleotide base pairings. Exception made for a partial relation with CSFV-a2, S171 was divergent from all other CSFV types showing divergence values from 10 to 15 . Divergence percentage with CSFV-a2 was $62.5 \%$, with a divergence mean value of 9.87 , resulting related, but belonging 
to a separate cluster. In addition, it was significantly less related to BDV species, a known characteristic of other CSFV strains (Figure 6 \& Figure 7). Base pairings comparison between strain S171 and strains of the different BDV genotypes scored high divergence values, ranging from 17 to 25 , with a mean value of 20.31. These values were very high in confront of the mean divergence value of 14.57 obtained comparing the other CSFV strains with BDV strains. Divergence from BDV-2 tentative species resulted also higher with CSFV-d (divergence values ranging from 21 to 22 ; mean divergence value 21.33), in confront of scores obtained with other CSFV genotypes CSFV-a, CSFV-b and CSFV-c (divergence values ranging from 15 to 20; mean divergence value 17.06). No relation was observed between CFSV and any other Pestivirus species (Figure 7). Other CSFV bovine strains have been reported from India and China, contaminants or associated to natural infection with clinical signs [8] [11] [45]. Indian strains, reported from the states of Tamil Nadu and Meghalaya, were genetically closely related to CSFV genotype a1 and a5 strains, respectively. However, a $G^{\star} U$ pairing in V1/3 was peculiar only in the Indian bovine strains from Tamil Nadu. In other CSFV strains this position is characterized by a conserved A-U pairing, while $\mathrm{G}-\mathrm{C}$ or $\mathrm{G}^{*} \mathrm{U}$ is conserved among BVDV-1, BVDV-2 and BVDV-3 strains (only 5 strains show exceptions, out of 1073 considered sequences).

\section{Discussion}

Different genetic characteristics were specific to Asian clusters in different

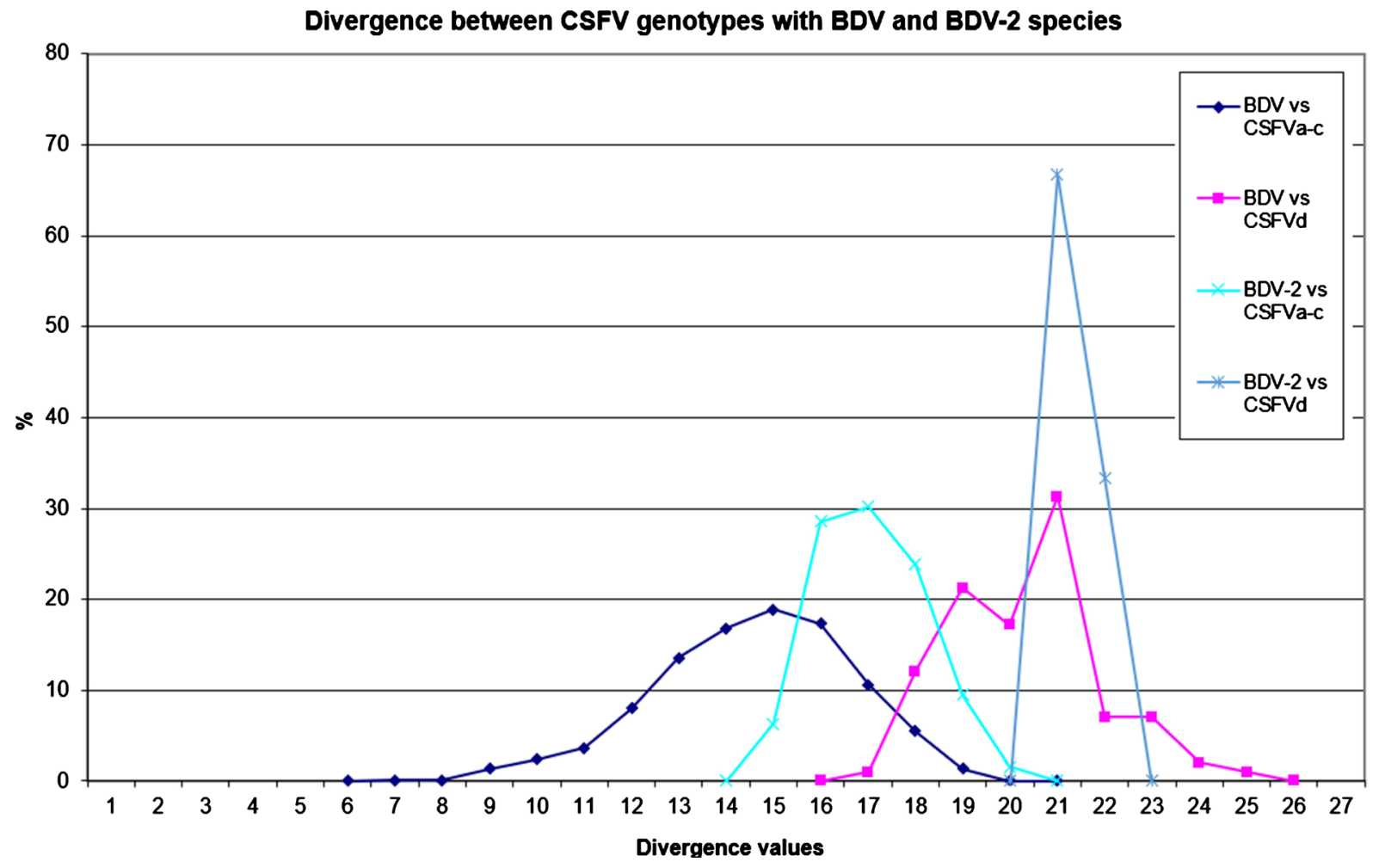

Figure 6. Graphical representation of PNS quantitative evaluation. Genotype CSFV-d is not related with BDV or BDV-2. 
Divergence between CSFV and other species

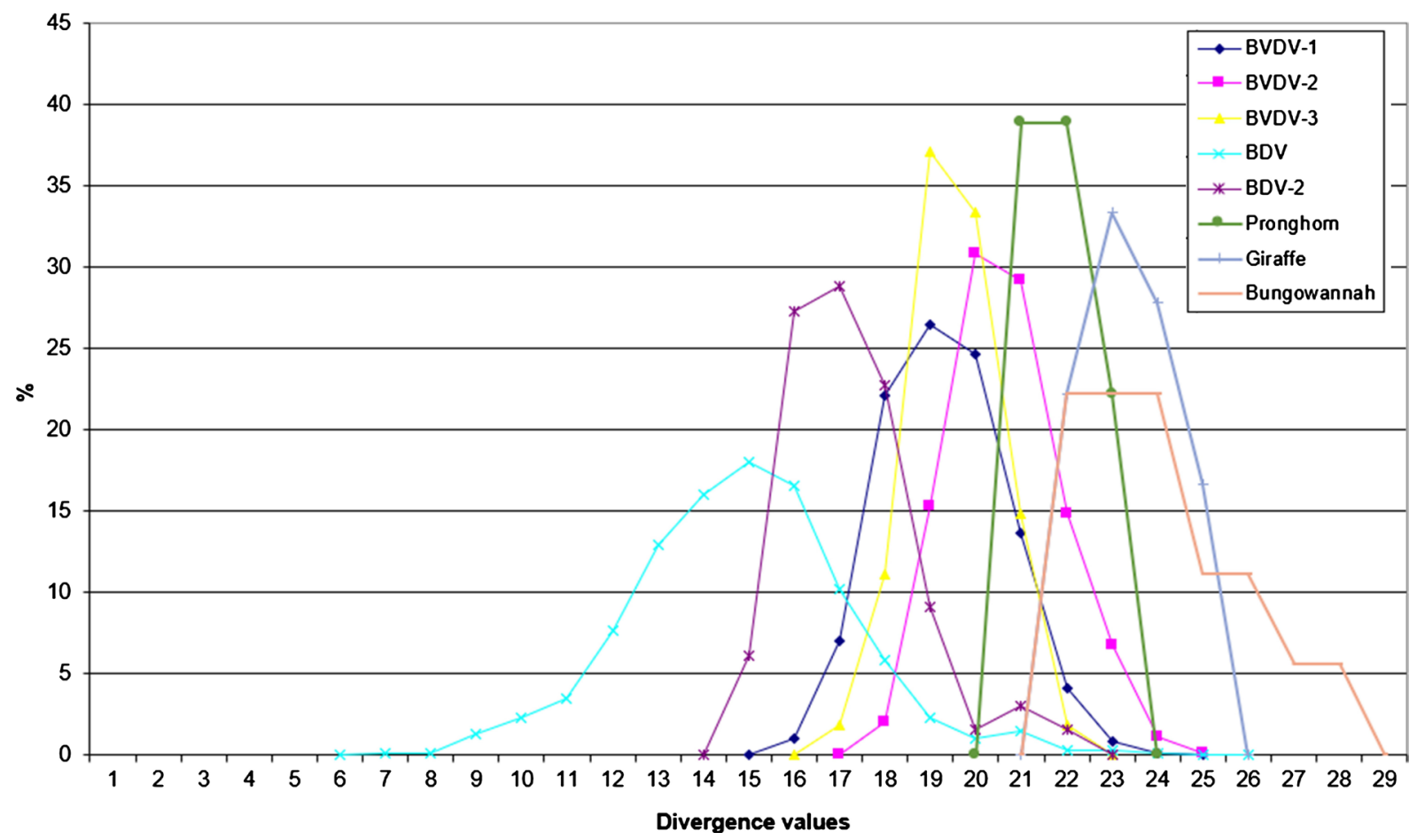

Figure 7. Graphical representation of PNS quantitative evaluation. CSFV is more related with BDV, showing higher relation with BDV than with BDV-2.

Pestivirus species, showing great potential in the evolution of the genus. Until 1997, only two BVDV-1 genotypes were known, 1a and 1b. Thanks to the development of virologic procedures and the increased interest in the pathogen, different new genomic types have been described. However, the heterogeneity of the species become even more evident in particular by the contribution of scientists from China and other Asian countries. BVDV-1 is known to have a cosmopolitan diffusion, but genotype geographic distribution may vary considerably. For example, while predominant in UK [46], in China, BVDV-1a genotype is rare, first reported in cattle in 2010 [5] and in pigs in 2012 [32], and shows also heterogeneity, suggesting that the virus have the great variation in evolution between the different host species [5]. BVDV-1 species resulted heterogeneous especially in China. The number of different BVDV-1 genotypes increased progressively. BVDV-1b and $1 \mathrm{~m}$ (PNS 1.15) are predominant [7]. Currently, BVDV-1 is represented by 12 types. Only in Italy, genetic diversity of BVDV-1 is higher, accounting for 14 different types [15]. Despite, the first description of the species in 1980 was related to $1 \mathrm{~b}$ infected cattle imported from Europe [10] [47], some of the genetic variants further described appeared to be restricted to China. For example, the phylogenetic reconstructions indicated that the clustering of the Chinese BVDV-1m genotype in the phylogenetic tree is a result of geographic isolation [7]. The Chinese strain ZM-95 for many years was the only representative of a separate cluster in the species, suggesting the circulation of a rare Pestivirus restricted in pigs. The strain was the first BVD virus isolated in 
China (Inner Mongolia) from pigs showing clinical symptoms and pathological lesions resembling mild classical swine fever [48], and characterized as genotype M [49] (PNS BVDV-1.15). Zhu et al. [10] reported a noncytopathic strain, genetically closely related to the pig strain ZM-95, associated with a disease characterized by high morbidity and mortality, a first BVDV- $1 \mathrm{~m}$ virus originated from cattle. In the present study, many other closely related strains have been considered from recent reports from China. Only the bovine strains BJ11_01 and S73 isolated in China in 2011 and 2013, 16 - 18 years later (Zhang et al., unpublished) [8], shared all the nucleotide characteristics of ZM-95, and all the other newly reported strains clarified genotype identification parameters. The genotype 1.15 resulted the most commonly diffused in China, and not restricted in pigs but circulating in cattle, buffaloes, yaks and Bactrian camels. The yak strain M31182 (Sun et al., unpublished) showed also characteristic nucleotide base pairs indicating radical sequence variations generating new genotype in the BVDV-1 species. Reported as genotype U, PNS 1.21, the genotype was identified also in other Chinese bovine and buffalo sequences.

The heterogeneity revealed in the BDV species was among the highest observed among pestiviruses. Only BVDV-1 accounted for more genotypic variants [14]. Sequence characteristics of Turkish and Chinese strains were highly divergent from other genogroups, indicating geographic segregation. HoBi-like pestiviruses (BVDV-3) was less heterogenous, but characterized by three Asian lineages. Strains from India and Bangladesh [12] [13] showed genetic diversity and molecular characteristics clearly distinct from those previously reported circulating globally, from bovine fetal serum or naturally infected cattle and buffaloes in America, Europe, Thailand and Australia, highlighting the independent evolution of the species in the Indian subcontinent. Similarly, the CSFV species showed low heterogeneity. However, two Asian genotypes were highly divergent. According to primary sequence analysis, the strain Kanagawa/74 (PNS genotype CSFV-c) was divergent in the species [50] [51], and proposed as harmonized outgroup strain for phylogenetic analyses [52]. This suggests that strain S171 (PNS genotype CSFV-d) should be considered as another outgroup of the species with even more enhanced divergence, with atypical characteristics, possibly related to host species adaptation or a result of geographic isolation, occurrences suggested also for BVDV-1 isolates in China [5] [7]. During a survey for the detection and genetic characterization of 5'-UTR and E2 gene of CSFV from bovine population of the northeastern region of Indian state of Meghalaya, out of 134 cattle serum samples tested, all were positive in reverse transcription-polymerase chain reaction (RT-PCR) for 5'-UTR region and 10 samples were positive for CSFV antigen by a commercial antigen capture enzyme linked immunosorbent assay (Ag-ELISA) [45]. Full length E2 region of CSFV were amplified from two positive samples, CS/ML/911/IDP/13 [KY860532] and CS/ML/AF/Umiam/14 [KY860531]. Phylogenetic analysis showed similarity with isolates reported from the neighboring state of Assam, as the pig strain 
IND/AS/GHY/G4 [KM362426] [53]. According to E2 region primary sequence analysis, these strains were clustered in the CSFV sub-genogroup 2.1 [45] [53]. The E2 regions of the bovine strains CS/ML/911/IDP/13 [KY860532] and CS/ML/AF/Umiam/14 [KY860531] showed 99\% nucleotide identity with IND/AS/GHY/G4 (PNS CSFV-a5) and 92\% with strain Paderborn [GQ902941] (PNS CSFV-a2). 5'-UTR evaluation by PNS from complete genome of Paderborn and IND/AS/GHY/G4 suggested the appurtenance of both bovine strains CS/ML/911/IDP/13 and CS/ML/AF/Umiam/14 to PNS CSFV-a5. As PNS CSFV-a4 (type Parambi), also this cluster was characteristic to India.

The high level of heterogeneity was reflected also in a certain confusion in the nomenclature of types. For example, the Chinese camel isolate 9, clustered as $M$ [31], diverged of only $2 \mathrm{bp}$ from the strain AQGN96BI5 of genotype $1.7(\mathrm{O})$, and $4 \mathrm{bp}$ from the 1.7 reference strain IS25CP/01 [28], thus justifying the reallocation in this BVDV group. Similarly, the Chinese camel isolate 5, reported as M [31] was reclustered in the present study, showing relation with genotype 1.17 (P) reference strain TJ06 [7] (divergence $6 \mathrm{bp}$ ). Confusion was also due to the use of same names to define different genetic clusters, as in the case of strains TR-2007-A-2368MS, TR-2007-Gu-175454-4695 [33], TR1, TR16 and TR72 [34], clustered as genotype L, reported from cattle in Turkey, genetically distant from homonymous isolates from continental Europe [54] [55] [56].

It is unlikely that geographic specificity of some types might be due to sampling bias, taking into account that strains have been reported from qualified laboratories, often identifying in the same epidemiological survey typical isolates. In certain cases, observations have been further validated at the level of the International Committee on Taxonomy of Viruses. For example, atypical ovine Turkish isolates BDV/Aydin/04-TR and BDV/Burdur/05-TR [57], previously considered a separate Pestivirus species [58], a new BDV subgroup [57] or BDV borderline candidate for reclustering as new species [59], have been recently classified as new genus Pestivirus member species (Pestivirus I) [1]. Furthermore, it is highly probable that future investigations will reveal the existence of new types in the genus since most of routinely conducted diagnostic work is based on serology and only few isolates are reported from certain regions. Similarly, despite evidence of Pestivirus seropositive animals in a large number of wild animal species [60], the number of reported isolates is very limited and related to few species as deer (Cervus elaphus) and roe deer (Capreolus capreolus) [61] [62], or the Japanese serow (Capricornis crispus) [63], Pyrenean chamois (Rupicapra pyrenaica) [64], Alpine chamois (Rupicapra rupicapra) [65], and captive mousedeer (Tragulus javanicus) [66], wisent (Bison bonasus) and reindeer (Rangifer tarandus) [67].

Also investigations in man, may reveal new aspects of Pestivirus epidemiology. Using mass spectrometry proteomics to analyze protein extracts from three Zika positive brains of deceased babies with severe brain lesions and arthrogryposis, peptides from the polyprotein of a Bovine-like viral diarrhea virus have 
been detected, suggesting that Zika virus may not be the only etiological agent responsible for microcephaly [68]. During the outbreak in Paraíba, in 2015, BVDV RNA was also found in the amniotic fluid collected from four mothers with babies affected by Zika and microcephaly. Two 5'-UTR RNA genomic sequences, have been provided by Prof Tanuri, Federal University of Rio de Janeiro, for further secondary structure analysis. The strain $4 \mathrm{p}$, considered in the present study, belonged to genotype BVDV-2b, typical genotype circulating in South America, genetically close to Brazilian strains LV56-1013 [69] and LVPatol0209 (Silveira et al., unpublished), but showing unreported variants in the IRES. The second isolate (12p) was a BVDV-1b1, genotype of cosmopolitan diffusion, very similar to bovine strains UEL9-BR/11 (Rodrigues et al., unpublished), isolated in Brazil in 2011, about 10 years later than the three other closely genetically related strains $133 / 02,4092 / 00$ and 3310/01 [70] previously identified in Spain in 2002. The human strain 12p showed also homology (98\% nucleotide identity) with four contaminant strains isolated in Mexico in 2012, the BVDV-1b1 NGR2, NGR3, NGR11 and NGR12 (Gomez-Romero et al., unpublished). The only strain previously reported in humans belonged to genotype BVDV-1.3 [71], another cluster with cosmopolitan diffusion.

Observed heterogeneity in the Pestivirus species has to be considered for potential implications on diagnostic tests, control and preventive measures, since commonly available tests and vaccines are based on viral antigenic substrate [72]. Due to possible geographical segregation, recognition of the molecular characteristics of field strains present in a population is fundamental for the control or eradication programs design, vaccine development or retracing infection sources in case of outbreaks [46] [73]. Laboratory testing difficulties may be due to serological cross-reactivity or divergence among pestiviruses. Serological surveillance of BVDV by ELISA does not distinguish between BVDV and BDV as source of infection. During routine genetic typing of pestiviruses in India, BDV was detected in sheep by real time RT-PCR [40]. All the samples yielded positive virus isolates in cell culture but were found negative by a BVDV antigen ELISA, suggesting that for diagnosis of BDV infection, the commercial BVDV Ag-ELISA should be used with caution. For adequate differentiation between BVDV and BDV, cross-serum neutralization test procedure has been recently developed [74]. Current BVDV diagnostic tests may fail to detect HoBi-like viruses or to differentiate between BVDV and HoBi-like viruses [75]. Furthermore, available commercial serological tests for BVDV do not reliably detect HoBi-like virus exposure, and cross protection against HoBi-like viruses conferred by current BVDV vaccines is likely limited [75]. Therefore, accurate genetic analysises are necessary for epidemiological studies, traceability and characterization of atypical strains.

The PNS method allowed an exhaustive representation of genomic variants in the genus, based on the comparative evaluation of about 1,400 Pestivirus strain sequences. The particularity of the PNS method is the exclusive consideration of 
strategic genomic sequences corresponding to the 5'-UTR IRES. Thus, at this level stable nucleotide variations assume high importance in terms of virus evolutionary history. Theoretically, applicable for the evaluation of all positive polarity RNA viruses, the PNS method was used for the determination of genotypes of the human hepatitis $\mathrm{C}$ virus [76], considering the unambiguous functional and genomic similitude between the two genera [77]. PNS sequence characterization easily discriminated species as Giraffe, Pronghorn or Bungowannah [78] [79]. Even if E2 glycoprotein gene is preferred in recent taxonomy of the species [80], CSFV was evaluated with 5'-UTR, and otherwise not comparable with 5'-UTR sequences of the other different species. Despite a limiting factor for differentiation of closely related isolates [80], short length target fragments in other regions of the viral genome as 5'-UTR or NS5B have been largely applied for genotyping purposes and molecular epidemiology [50] [52] [81]. In addition, according to the Terrestrial Manual of the World Organization for Animal Health (Chapter 2.8.3 CSFV; Paragraph 1.1.5.) [82], the 5'-UTR of the CSFV genome (150 nucleotides) is also foreseen for genetic typing and molecular epidemiology of CSF. However, in light of the movement towards whole genome sequencing and recent species typing [1], the routine work performed in veterinary laboratories in the future will possibly be based on full-length genome comparison to delineate Pestivirus genogroups and species, despite still problematic due to lack of a sufficient number of sequences representing existing genetic variants. For example, current classification of BVDV isolates is generated using short genome fragments, giving that $97.7 \%$ of deposited sequences are restricted to 5'-UTR or Npro, mainly [16].

In addition to accurate virologic investigations, it is important to understand ways of diffusion and in particular prevent spread of atypical genetic clusters. The major concern is represented by the fact that if genetically related types induce effective cross immunity, at the contrary, divergent genetically atypical types might pose problems in terms of diagnostic reliable detection or vaccine conferred cross protection against such viruses. In addition, even non-naïve local animal populations might be more exposed in case of heterogeneous strains due to non-sufficient cross immunity. Trade of live animals is generally considered the main route of pathogen diffusion. For example, in UK, only three BVDV-1 types were detected in 1999. In 2013, phylogenetic analysis demonstrated the existence of six genotypes of BVDV-1 circulating in the country. This indicated that restocking of cattle from continental Europe has increased the genetic diversity [46]. Unless illegal animal movements occur, trade is the relatively easier controllable and preventable among ways of introduction of the virus in free herds or free areas. Contamination of biological products and iatrogenic infection are probably also relevant aspects of the virus epidemiology. This recalls also the risk of spreading exotic strains, requiring not only controls on live animals, animal products, semen, ova and embryos, but also potentially contaminated biological products. Eighty-three strains contaminant of biological 
products, considered in the present study, were reported mainly from Asian countries. Commercialized batches of BVDV-1 contaminated bovine serum have been reported also in China [8]. The contamination of fetal bovine serum (FBS) as a vehicle for the rapid spread of the pathogen is a known and not really infrequent phenomenon [29] [83]. This might be the source of introduction of BVDV-3 in Italy, first identified in Europe in FBS imported from Brazil [84], and further reported in Italian cattle herds with respiratory distress and reproductive failures [85] [86] [87]. A similar hypothesis could be formulated for the BVDV-1 genotype 1.21. Some characteristic Asian strains of the genotype 1.21 have been shown to circulate also in southern Italy [88], suggesting ways of diffusion other than direct contact among animals. Being unlikely the direct import of live animals from China, other factors were implicated. The strain 441/09, isolated in 2009 in Puglia region, showed high similarity with the originally reported Chinese yak strain. The other two strains, 130/15-4215 and 130/15-5364, have been isolated six years later in 2015 in Sicily. Their sequences were very similar to each other. When compared to other BVDV-1 genotypes, both strains resulted divergent from most of other types, with high divergence values as 20 bp with the European genotype 1.22. However, the lowest divergence values were obtained with strains belonging to genotype 1.21 , justifying allocation in the cluster, but in a separate sub genotype 1.21.1. These observed genomic variations were coherent with virus evolutionary adaptation related to spatial temporal factors. Strain 441/09 may represent the evidence of the introduction of an exotic Pestivirus variant in the animal population in Italy, while strains 130/15-4215 and 130/15-5364 may represent the result of the virus adaptational evolution in the new environment, after an adequate period of time. This suggest the introduction of genotype 1.21 from China in 2009 and the potential risk of the endemic status of the virus in the area, supported by evidence of genomic characteristics mutations, possibly related to geographical situation or contact with virus populations circulating in the region. Interestingly, the Italian bovine strain SI/207/12 [89], reported as genotype T, was clustered in the genotype 1.6, scoring low divergence values with most of the strains in the group, all reported as genotype BVDV-1n, essentially of Asian origin, including the Japanese strains so CP/75 [90] and Shitara/02/06 [91] or the South Korean strains 06z71, 06z127 (Yang and Kweon, unpublished) and KB01 (Oem et al., unpublished).

Other ways of diffusion may be revealed by the unusual circulation of CSFV in animal host different from suids. CSFV is generally considered to be restricted to domestic and wild suids. Current knowledge indicates that experimental infections without clinical signs have been reported in cattle, sheep, goats and deer, but there is no evidence that these species become infected in nature [92] [93] [94] [95]. Only in one case, hog cholera virus has been identified in sheep [70]. In Spain, the reported 5440/99 ovine strain showed sequence similarities with CSFV vaccine strains used in pigs, suggesting spillovers from prophylactic campaigns. Another ovine isolate (strain 12 Ovine liver 113nt) [96], reported 
from United Arab Emirates in the framework of investigations for pestiviruses, was suspected as CSFV. CSFV bovine strains detected in China might find their origin from vaccine prophylaxis performed in domestic animal populations [11]. Anti BVDV killed virus vaccines are available in China. However, for long time there were no commercial BVDV vaccines in the Chinese market. In cattle and yaks, BVDV was prevented by the wide application of triple dose of the live attenuated hog cholera lapinized vaccine (HCLV) strain in different areas of China, and especially in Tibet and Qinghai provinces. Since previous experimental studies conducted by Yuan Qingzhi in 1957 on HCLV, the only one CSFV authorized vaccine in China, efficacy and safety of the prophylactic use for BVDV in lactating and pregnant cows, calves and yaks have been demonstrated [97]. In Tibet, the immunization with HCLV of bovine species resulted beneficial in reducing losses due to BVDV infection. However, long-term use of live attenuated HCLV gave the opportunity for vaccinal strains adaptation in cattle, with further natural diffusion as possible consequence.

\section{Conclusion}

In the present study, according to secondary structure analysis, the genus Pestivirus resulted heterogeneous. Sequence characteristics among Asian genomic clusters within the different Pestivirus species suggested geographic segregation and occurrence of micro-evolutive steps in the genus evolutionary history. This aspect was particularly evident in atypical sequences originated from China or Turkey, indicating risk of diffusion by animals and products trade or contamination of biological products as bovine calf serum, with potential diagnostic and control difficulties. Determination of species heterogeneity is important for diagnostic efficiency and prophylactic purposes, taking into account possible wider animal host range by different Pestivirus species, causing overlapping clinical features, potential influence on eradication programs, and eventual regulatory measures.

\section{Conflicts of Interest}

The authors declare no conflicts of interest regarding the publication of this paper.

\section{References}

[1] International Committee on Taxonomy of Viruses (2017) Virus Taxonomy. 10th Report 2017 Release EC 49, Singapore, July 2017.

[2] Deng, M., Ji, S., Fei, W., Raza, S., He, C., Chen, Y., Chen, H. and Guo, A. (2015) Prevalence Study and Genetic Typing of Bovine Viral Diarrhea Virus (BVDV) in Four Bovine Species in China. PLOS ONE, 10, e0121718.

https://doi.org/10.1371/journal.pone.0121718

[3] Gao, S., Du, J., Shao, J., Lang, Y., Lin, T., Cong, G., Zhao, F., Belak, S., Liu, L., Chang, H. and Yin, H. (2014) Genome Analysis Reveals a Novel Genetically Divergent Subgenotype of Bovine Viral Diarrhea Virus in China. Infection, Genetics and Evolution, 21, 489-491. https://doi.org/10.1016/j.meegid.2013.06.010 
[4] Gong, X., Cao, X., Zheng, F., Chen, Q., Zhou, J., Yin, H., Liu, L. and Cai, X. (2013) Identification and Characterization of a Novel Subgenotype of Bovine Viral Diarrhea Virus Isolated from Dairy Cattle in Northwestern China. Virus Genes, 46, 375-376. https://doi.org/10.1007/s11262-012-0861-3

[5] Wang, W., Shi, X., Tong, Q., Wu, Y., Xia, M.Q., Ji, Y., Xue, W. and Wu, H. (2014) A Bovine Viral Diarrhea Virus Type 1a Strain in China: Isolation, Identification, and Experimental Infection in Calves. Virology Journal, 11, 8. https://doi.org/10.1186/1743-422X-11-8

[6] Weng, X.G., Song, Q.J., Wu, Q., Liu, M.C., Wang, M.L. and Wang, J.F. (2015) Genetic Characterization of Bovine Viral Diarrhea Virus Strains in Beijing, China and Innate Immune Responses of Peripheral Blood Mononuclear Cells in Persistently Infected Dairy Cattle. Journal of Veterinary Science, 16, 491-500.

https://doi.org/10.4142/jvs.2015.16.4.491

[7] Xue, F., Zhu, Y.M., Li, J., Zhu, L.C., Ren, X.G., Feng, J.K., Shi, H.F. and Gao, Y.R. (2010) Genotyping of Bovine Viral Diarrhea Viruses from Cattle in China between 2005 and 2008. Veterinary Microbiology, 143, 379-383. https://doi.org/10.1016/j.vetmic.2009.11.010

[8] Zhang, S.Q., Tan, B., Guo, L., Wang, F.X., Zhu, H.W., Wen, Y.J. and Cheng, S. (2014) Genetic Diversity of Bovine Viral Diarrhea Viruses in Commercial Bovine Serum Batches of Chinese Origin. Infection, Genetics and Evolution, 27, 230-233. https://doi.org/10.1016/j.meegid.2014.07.021

[9] Zhong, F., Li, N., Huang, X., Guo, Y., Chen, H., Wang, X., Shi, C. and Zhang, X. (2011) Genetic Typing and Epidemiologic Observation of Bovine Viral Diarrhea Virus in Western China. Virus Genes, 42, 204-207. https://doi.org/10.1007/s11262-010-0558-4

[10] Zhu, L., Lu, H., Cao, Y., Gai, X., Guo, C., Liu, Y., et al. (2016) Molecular Characterization of a Novel Bovine Viral Diarrhea Virus Isolate SD-15. PLoS ONE, 11, e0165044. https://doi.org/10.1371/journal.pone.0165044

[11] Giangaspero, M., Kumar, S.K. and Zhang, S.Q. (2017) Classical Swine Fever Virus in Cattle. Veterinary Record, 181, 73.

[12] Haider, N., Rahman, M.S,, Khan, S.U., Mikolon, A., Gurley, E.S., Osmani, M.G., Shanta, I.S., Paul, S.K., Macfarlane-Berry, L., Islam, A., Desmond, J., Epstein, J.H., Daszak, P., Azim, T., Luby, S.P., Zeidner, N. and Rahman, M.Z. (2014) Identification and Epidemiology of a Rare HoBi-Like Pestivirus Strain in Bangladesh. Transboundary and Emerging Diseases, 61, 193-198. https://doi.org/10.1111/tbed.12218

[13] Mishra, N., Rajukumar, K., Pateriya, A., Kumar, M., Dubey, P., Behera, S.P., Verma, A., Bhardwaj, P., Kulkarni, D.D., Vijaykrishna, D. and Reddy, N.D. (2014) Identification and Molecular Characterization of Novel and Divergent HoBi-Like Pestiviruses from Naturally Infected Cattle in India. Veterinary Microbiology, 174, 239-246. https://doi.org/10.1016/j.vetmic.2014.09.017

[14] Giangaspero, M. and Apicella, C. (2018) Bovine Viral Diarrhea Virus Type 1 Current Taxonomy According to Palindromic Nucleotide Substitutions Method. Journal of Virological Methods, 256, 37-76. https://doi.org/10.1016/j.jviromet.2018.02.003

[15] Giangaspero, M., Yesilbag, K. and Apicella, C. (2018) Who's Who in the Bovine Viral Diarrhea Virus Type 1 Species: Genotypes L and R. Virus Research, 256, 50-75. https://doi.org/10.1016/j.virusres.2018.07.009

[16] Yesilbag, K., Alpay, G. and Becher, P. (2017) Variability and Global Distribution of Subgenotypes of Bovine Viral Diarrhea Virus. Viruses, 9, pii: E128. 
[17] Giangaspero, M. and Harasawa, R. (2007) Numerical Taxonomy of Genus Pestivirus Based on Palindromic Nucleotide Substitutions in the 5' Untranslated Region. Journal of Virological Methods, 146, 375-388. https://doi.org/10.1016/j.jviromet.2007.07.009

[18] Harasawa, R. and Giangaspero, M. (1998) A Novel Method for Pestivirus Genotyping Based on Palindromic Nucleotide Substitutions in the 5'-Untranslated Region. Journal of Virological Methods, 70, 225-230. https://doi.org/10.1016/S0166-0934(97)00180-8

[19] Deng, R. and Brock, K.V. (1993) 5' and 3' Untranslated Regions of Pestivirus Genome: Primary and Secondary Structure Analyses. Nucleic Acids Research, 21, 1949-1957. https://doi.org/10.1093/nar/21.8.1949

[20] Harasawa, R. (1994) Comparative Analysis of the 5' Non-Coding Region of Pestivirus RNA Detected from Live Virus Vaccines. The Journal of Veterinary Medical Science, 56, 961-964.

[21] Willcocks, M.M., Zaini, S., Chamond, N., Ulryck, N., Allouche, D., Rajagopalan, N., Davids, N.A., Fahnøe, U., Hadsbjerg, J., Rasmussen, T.B., Roberts, L.O., Sargueil, B., Belsham, G.J. and Locker, N. (2017) Distinct Roles for the IIId2 Sub-Domain In Pestivirus and Picornavirus Internal Ribosome Entry Sites. Nucleic Acids Research, 45, 13016-13028. https://doi.org/10.1093/nar/gkx991

[22] Zuker, M. and Stiegler, P. (1981) Optimal Computer Folding of Large RNA Sequences Using Thermodynamics and Auxiliary Information. Nucleic Acids Research, 9, 133-148. https://doi.org/10.1093/nar/9.1.133

[23] Freier, S.M., Kierzek, R., Jaeger, J.A., Sugimoto, N., Caruthers, M.H., Nielson, T. and Turner, D.H. (1986) Improved Free-Energy Parameters for Predictions of RNA Duplex Stability. Proceedings of the National Academy of Sciences of the United States of America, 83, 9373-9377. https://doi.org/10.1073/pnas.83.24.9373

[24] Giangaspero, M. and Apicella, C. (2014) Improved Palindromic Nucleotide Substitutions Software Version 2.0. Genotyping Based on the Secondary Structure Alignment in the 5 Untranslated Region of Pestivirus RNA. Journal of Bioinformatics and Intelligent Control, 3, 39-64. https://doi.org/10.1166/jbic.2014.1064

[25] Chenna, R., Sugawara, H., Koike, T., Lopez, R., Gibson, T.J., Higgins, D.G. and Thompson, J.D. (2003) Multiple Sequence Alignment with the Clustal Series of Programs. Nucleic Acids Research, 31, 3497-3500. https://doi.org/10.1093/nar/gkg500

[26] Saitou, N. and Nei, M. (1987) The Neighbor-Joining Method: A New Method for Reconstructing Phylogenetic Trees. Molecular Biology and Evolution, 4, 406-425.

[27] Kumar, S., Stecher, G. and Tamura, K. (2016) MEGA7: Molecular Evolutionary Genetics Analysis Version 7.0 for Bigger Datasets. Molecular Biology and Evolution, 33, 1870-1874. https://doi.org/10.1093/molbev/msw054

[28] Nagai, M., Hayashi, M., Itou, M., Fukutomi, T., Akashi, H., Kida, H. and Sakoda, Y. (2008) Identification of New Genetic Subtypes of Bovine Viral Diarrhea Virus Genotype 1 Isolated in Japan. Virus Genes, 36, 135-139.

https://doi.org/10.1007/s11262-007-0190-0

[29] Xia, H., Vijayaraghavan, B., Belak, S. and Liu, L. (2011) Detection and Identification of the Atypical Bovine Pestiviruses in Commercial Foetal Bovine Serum Batches. PLoS ONE, 6, e28553. https://doi.org/10.1371/journal.pone.0028553

[30] Harasawa, R. and Mizusawa, H. (1995) Demonstration and Genotyping of Pestivirus RNA from Mammalian Cell Lines. Microbiology and Immunology, 39, 979-985. https://doi.org/10.1111/j.1348-0421.1995.tb03301.x 
[31] Gao, S., Luo, J., Du, J., Lang, Y., Cong, G., Shao, J., Lin, T., Zhao, F., Belak, S., Liu, L., Chang, H. and Yin, H. (2013) Serological and Molecular Evidence for Natural Infection of Bactrian Camels with Multiple Subgenotypes of Bovine Viral Diarrhea Virus in Western China. Veterinary Microbiology, 163, 172-176. https://doi.org/10.1016/j.vetmic.2012.12.015

[32] Deng, Y., Sun, C.Q., Cao, S.J., Lin, T., Yuan, S.S., Zhang, H.B., Zhai, S.L., Huang, L., Shan, T.L., Zheng, H., et al. (2012) High Prevalence of Bovine Viral Diarrhea Virus 1 in Chinese Swine Herds. Veterinary Microbiology, 159, 490-493. https://doi.org/10.1016/j.vetmic.2012.04.023

[33] Oguzoglu, T.C., Muz, D., Yilmaz, V., Timurkan, M.O., Alkan, F., Akca, Y. and Burgu, I. (2012) Molecular Characteristics of Bovine Virus Diarrhoea Virus 1 Isolates from Turkey: Approaches for an Eradication Programme. Transboundary and Emerging Diseases, 59, 303-310. https://doi.org/10.1111/j.1865-1682.2011.01272.x

[34] Yesilbag, K., Förster, C., Ozyigit, M.O., Alpay, G., Tuncer, P., Thiel, H.-J. and König, M. (2014) Characterisation of Bovine Viral Diarrhoea Virus (BVDV) Isolates from an Outbreak with Haemorrhagic Enteritis and Severe Pneumonia. Veterinary Microbiology, 169, 42-49. https://doi.org/10.1016/j.vetmic.2013.12.005

[35] Mao, L., Li, W., Zhang, W., Yang, L. and Jiang, J. (2012) Genome Sequence of a Novel Hobi-Like Pestivirus in China. Journal of Virology, 86, 12444. https://doi.org/10.1128/JVI.02159-12

[36] Liu, L., Kampa, J., Belak, S. and Baule, C. (2009) Virus Recovery and Full-Length Sequence Analysis of Atypical Bovine Pestivirus Th/04_KhonKaen. Veterinary Microbiology, 138, 62-68. https://doi.org/10.1016/j.vetmic.2009.03.006

[37] Li, W., Mao, L., Zhao, Y., Sun, Y., He, K. and Jiang, J. (2013) Detection of Border Disease Virus (BDV) in Goat Herds Suffering Diarrhea in Eastern China. Virology Journal, 10, 80. https://doi.org/10.1186/1743-422X-10-80

[38] Leskova, V., Jackova, A., Vlasakova, M. and Vilcek, S. (2013) Genetic Characterization of a Border Disease Virus Isolate Originating from Slovakia. Acta Virologica, 57, 17-25. https://doi.org/10.4149/av_2013_01_17

[39] Toplu, N., Oguzoglu, T.C. and Albayrak, H. (2012) Dual Infection of Fetal and Neonatal Small Ruminants with Border Disease Virus and Peste des Petits Ruminants Virus (PPRV): Neuronal Tropism of PPRV as a Novel Finding. Journal of Comparative Pathology, 146, 289-297. https://doi.org/10.1016/j.jcpa.2011.07.004

[40] Mishra, N., Rajukumar, K., Vilcek, S., Kalaiyarasu, S., Behera, S.P., Dubey, P., Nema, R.K., Gavade, V.B., Dubey, S.C. and Kulkarni, D.D. (2016) Identification and Molecular Characterization of Border Disease Virus (BDV) from Sheep in India. Comparative Immunology, Microbiology \& Infectious Diseases, 44, 1-7. https://doi.org/10.1016/j.cimid.2015.11.001

[41] Lin, Y.J., Chien, M.S., Deng, M.C. and Huang, C.C. (2007) Complete Sequence of a Subgroup 3.4 Strain of Classical Swine Fever Virus from Taiwan. Virus Genes, 35, 737-744. https://doi.org/10.1007/s11262-007-0154-4

[42] Bhaskar, N., Ravishankar, C., Rajasekhar, R., Sumod, K., Sumithra, T.G., John, K., Mini, M., Ravindran, R., Shaji, S. and Aishwarya, J. (2015) Molecular Typing and Phylogenetic Analysis of Classical Swine Fever Virus Isolates from Kerala, India. VirusDisease, 26, 260-266. https://doi.org/10.1007/s13337-015-0271-y

[43] Harasawa, R. and Giangaspero, M. (1999) Genetic Variation in the 5' End and NS5B Regions of Classical Swine Fever Virus Genome among Japanese Isolates. Microbiology and Immunology, 43, 373-379. https://doi.org/10.1111/j.1348-0421.1999.tb02418.x 
[44] Sakoda, Y., Ozawa, S., Damrongwatanapokin, S., Sato, M., Ishikawa, K. and Fukusho, A. (1999) Genetic Heterogeneity of Porcine and Ruminant Pestiviruses Mainly Isolated in Japan. Veterinary Microbiology, 65, 75-86. https://doi.org/10.1016/S0378-1135(98)00284-3

[45] Chakraborty, A.K., Karam, A., Mukherjee, P., Barkalita, L., Borah, P., Das, S., Sanjukta, R., Puro, K., Ghatak, S., Shakuntala, I., Sharma, I., Laha, R.G. and Sen, A. (2018) Detection of Classical Swine Fever Virus E2 Gene in Cattle Serum Samples from Cattle Herds of Meghalaya. VirusDisease, 29, 89-95.

https://doi.org/10.1007/s13337-018-0433-9

[46] Booth, R.E., Thomas, C.J., El-Attar, L.M., Gunn, G. and Brownlie, J. (2013) A Phylogenetic Analysis of Bovine Viral Diarrhoea Virus (BVDV) Isolates from Six Different Regions of the UK and Links to Animal Movement Data. Veterinary Research, 44, 43. https://doi.org/10.1186/1297-9716-44-43

[47] Li, Y., Liu, Z. and Wu, Y. (1983) Isolation and Identification of Bovine Viral Diarrhea Virus-Mucosal Disease Virus Strain Changchun 184. Chinese Journal of Veterinary Science, 3, 546-553.

[48] Wang, X., Tu, C., Li, H., Jin, K., Xuan, H., Chang, G., Sun, H., Zhu, W., Fei, E. and Yin, Z. (1996) Detection and Isolation of Bovine Viral Diarrhea Virus from Classical Swine Fever Suspected Pigs. Chinese Journal of Veterinary Science, 16, 341-345.

[49] Xu, X., Zhang, Q., Yu, X., Liang, L., Xiao, C., Xiang, H. and Tu, C. (2006) Sequencing and Comparative Analysis of a Pig Bovine Viral Diarrhea Virus Genome. Virus Research, 122, 164-170. https://doi.org/10.1016/j.virusres.2006.05.005

[50] Beer, M., Goller, K.V., Staubach, C. and Blome, S. (2015) Genetic Variability and Distribution of Classical Swine Fever Virus. Animal Health Research Reviews, 16, 33-39. https://doi.org/10.1017/S1466252315000109

[51] Dreier, S., Zimmermann, B., Moennig, V. and Wilke, I.G. (2007) A Sequence Database Allowing Automated Genotyping of Classical Swine Fever Virus Isolates. Journal of Virological Methods, 140, 95-99. https://doi.org/10.1016/j.jviromet.2006.11.013

[52] Paton, D.J., McGoldrick, A., Greiser-Wilke, I., Parchariyanon, S., Song, J.Y., Liou, P.P., Stadejek, T., Lowings, J.P., Björklund, H. and Belák, S. (2000) Genetic Typing of Classical Swine Fever Virus. Veterinary Microbiology, 73, 137-157. https://doi.org/10.1016/S0378-1135(00)00141-3

[53] Ahuja, A., Bhattacharjee, U., Chakraborty, A.K., Karam, A., Ghatak, S., Puro, K., Das, S., Shakuntala, I., Srivastava, N., Ngachan, S.V. and Sen A. (2015) Complete Genome Sequence of Classical Swine Fever Virus Subgenogroup 2.1 from Assam, India. Genome Announcements, 3, e01437-14.

https://doi.org/10.1128/genomeA.01437-14

[54] Bachofen, C., Stalder, H., Braun, U., Hilbe, M., Ehrensperger, F. and Peterhans, E. (2008) Co-Existence of Genetically and Antigenically Diverse Bovine Viral Diarrhoea Viruses in an Endemic Situation. Veterinary Microbiology, 131, 93-102. https://doi.org/10.1016/j.vetmic.2008.02.023

[55] Jackova, A., Novackova, M., Pelletier, C., Audeval, C., Gueneau, E., Haffar, A., Petit, E., Rehby, L. and Vilcek, S. (2008) The Extended Genetic Diversity of BVDV-1: Typing of BVDV Isolates from France. Veterinary Research Communications, 32, 7-11. https://doi.org/10.1007/s11259-007-9012-z

[56] Luzzago, C., Lauzi, S., Ebranati, E., Giammarioli, M., Moreno, A., Cannella, V., Masoero, L., Canelli, E., Guercio, A., Caruso, C., Ciccozzi, M., De Mia, G.M., Acutis, P.L., Zehender, G. and Peletto, S. (2014) Extended Genetic Diversity of Bovine Viral 
Diarrhea Virus and Frequency of Genotypes and Subtypes in Cattle in Italy between 1995 and 2013. BioMed Research International, 2014, Article ID: 147145. https://doi.org/10.1155/2014/147145

[57] Oguzoglu, T.C., Tan, M.T., Toplu, N., Demir, A.B., Bilge-Dagalp, S., Karaoglu, T., Ozkul, A., Alkan, F., Burgu, I., Haas, L. and Greiser-Wilke, I. (2009) Border Disease Virus (BDV) Infections of Small Ruminants in Turkey: A New BDV Subgroup? Veterinary Microbiology, 135, 374-379. https://doi.org/10.1016/j.vetmic.2008.09.085

[58] Giammarioli, M., La Rocca, S.A., Steinbach, F., Casciari, C. and De Mia, G.M. (2011) Genetic and Antigenic Typing of Border Disease Virus (BDV) Isolates from Italy Reveals the Existence of a Novel BDV Group. Veterinary Microbiology, 147, 231-236. https://doi.org/10.1016/j.vetmic.2010.06.027

[59] Giangaspero, M. (2011) Genetic Variation of Border Disease Virus Species Strains. Veterinaria Italiana, 47, 415-435.

[60] Doyle, L.G. and Heuschele, W.P. (1983) Bovine Viral Diarrhea Virus Infection in Captive Exotic Ruminants. Journal of the American Veterinary Medical Association, 183, 1257-1259.

[61] Becher, P., Orlich, M., Kosmidou, A., Konig, M., Baroth, M. and Thiel, H-J. (1999) Genetic Diversity of Pestiviruses: Identification of Novel Groups and Implications for Classification. Virology, 262, 64-71. https://doi.org/10.1006/viro.1999.9872

[62] Frölich, K. and Hofmann, M. (1995) Isolation of Bovine Viral Diarrhea Virus-Like Pestiviruses from Roe Deer (Capreolus capreolus). Journal of Wildlife Diseases, 31, 243-246. https://doi.org/10.7589/0090-3558-31.2.243

[63] Harasawa, R., Aoyama, F., Goryo, M., Nishimura, T., Matsubara, K., Tsujimoto, T. and Giangaspero, M. (2006) Evidence for Pestivirus Infection in Free-Living Japanese Serows, Capricornis crispus. Microbiology and Immunology, 50, 817-821. https://doi.org/10.1111/j.1348-0421.2006.tb03858.x

[64] Arnal, M., Fernandez-de-Luco, D., Riba, L., Maley, M., Gilray, J., Willoughby, K., Vilček, S. and Nettleton, P.F. (2004) A Novel Pestivirus Associated with Deaths in Pyrenean chamois (Rupicapra pyrenaica pyrenaica). Journal of General Virology, 85, 3653-3657. https://doi.org/10.1099/vir.0.80235-0

[65] Casaubon, J., Vogt, H.R., Stalder, H., Hug, C. and Ryser-Degiorgis, M.P. (2012) Bovine Viral Diarrhea Virus in Free-Ranging Wild Ruminants in Switzerland: Low Prevalence of Infection Despite Regular Interactions with Domestic Livestock. BMC Veterinary Research, 8, 204. https://doi.org/10.1186/1746-6148-8-204

[66] Grondahl, C., Uttenthal, A., Houe, H., Rasmussen, T.B., Hoyer, M.J. and Larsen, L.E. (2003) Characterisation of a Pestivirus Isolated from Persistently Infected Mousedeer (Tragulus javanicus). Archives of Virolog, 148, 1455-1463. https://doi.org/10.1007/s00705-003-0130-9

[67] Giangaspero, M., Harasawa, R., Muschko, K. and Büttner, M. (2006) Characterisation of the 5'-Untranslated Region of Wisent (Bison bonasus) and Reindeer (Rangifer tarandus). Veterinaria Italiana, 42, 165-172.

[68] Nogueira, F.C.S., Velasquez, E., Melo, A.S.O. and Domont, G.B. (2016) Zika Virus May Not Be Alone: Proteomics Associates a Bovine-Like Viral Diarrhea Virus to Microcephaly. bioRxiv preprint.

[69] Weber, M.N., Silveira, S., Machado, G., Groff, F.H., Mosena, A.C., Budaszewski, R.F., Dupont, P.M., Corbellini, L.G. and Canal, C.W. (2014) High Frequency of Bovine Viral Diarrhea Virus Type 2 in Southern Brazil. Virus Research, 191, 117-124. https://doi.org/10.1016/j.virusres.2014.07.035

[70] Hurtado, A., Garcia-Perez, A.L., Aduriz, G. and Juste, R.A. (2003) Genetic Diversity 
of Ruminant Pestiviruses from Spain. Virus Research, 92, 67-73. https://doi.org/10.1016/S0168-1702(02)00315-5

[71] Giangaspero, M., Harasawa, R. and Verhulst, A. (1997) Genotypic Analysis of the 5'-Untranslated Region of a Pestivirus Strain Isolated from Human Leucocytes. Microbiology and Immunology, 41, 829-834. https://doi.org/10.1111/j.1348-0421.1997.tb01936.x

[72] Bolin, S.R., McClurkin, A.W., Cutlip, R.C. and Coria, M.F. (1985) Response of Cattle Persistently Infected with Noncytopathic Bovine Viral Diarrhea Virus to Vaccination for Bovine Viral Diarrhea and to Subsequent Challenge Exposure with $\mathrm{Cy}$ topathic Bovine Viral Diarrhea Virus. American Journal of Veterinary Research, 46, 2467-2470.

[73] Kuta, A., Polak, M.P., Larska, M. and Zmudzinski, J.F. (2013) Predominance of Bovine Viral Diarrhea Virus $1 \mathrm{~b}$ and 1d Subtypes during Eight Years of Survey in Poland. Veterinary Microbiology, 166, 639-644. https://doi.org/10.1016/j.vetmic.2013.07.002

[74] Kaiser, V., Nebel, L., Schüpbach-Regula, G., Zanoni, R.G. and Schweizer, M. (2017) Influence of Border Disease Virus (BDV) on Serological Surveillance within the Bovine Virus Diarrhea (BVD) Eradication Program in Switzerland. BMC Veterinary Research, 13, 21. https://doi.org/10.1186/s12917-016-0932-0

[75] Bauermann, F.V., Ridpath, J.F., Weiblen, R. and Flores, E.F. (2013) HoBi-Like Viruses: An Emerging Group of Pestiviruses. Journal of Veterinary Diagnostic Investigation, 25, 6-15. https://doi.org/10.1177/1040638712473103

[76] Prabdial-Sing, N., Giangaspero, M., Puren, A.J., Mahlangu, J., Barrow, P. and Bowyer, S.M. (2011) Palindromic-Nucleotide Substitutions (PNS) of Hepatitis C Virus Genotypes 1 and 5a from South Africa. Journal of Virological Methods, 175, 272-277. https://doi.org/10.1016/j.jviromet.2011.05.005

[77] Wang, C., Sarnow, P. and Siddiqui, A. (1993) Translation of Human Hepatitis C Virus RNA in Cultured Cells Is Mediated by an Internal Ribosome Binding Mechanism. Journal of Virology, 67, 3338-3344.

[78] Giangaspero, M. and Harasawa, R. (2011) Species Characterization in the Genus Pestivirus According to Palindromic Nucleotide Substitutions in the 5'-Untranslated Region. Journal of Virological Methods, 174, 166-172.

https://doi.org/10.1016/j.jviromet.2011.04.004

[79] Harasawa, R., Giangaspero, M., Ibata, G. and Paton, P.J. (2000) Giraffe Strain of Pestivirus. Its Taxonomic Status Based on the 5'-Untranslated Region. Microbiology and Immunology, 44, 915-921. https://doi.org/10.1111/j.1348-0421.2000.tb02583.x

[80] Postel, A., Schmeiser, S., Bernau, J., Meindl-Boehmer, A., Pridotkas, G., Dirbakova, Z., Mojzis, M. and Becher, P. (2012) Improved Strategy for Phylogenetic Analysis of Classical Swine Fever Virus Based on Full-Length E2 Encoding Sequences. Veterinary Research, 43, 50. https://doi.org/10.1186/1297-9716-43-50

[81] Lowings, P., Ibata, G., Needham, J. and Paton, D. (1996) Classical Swine Fever Virus Diversity and Evolution. Journal of General Virology, 77, 1311-1321. https://doi.org/10.1099/0022-1317-77-6-1311

[82] World Organisation for Animal Health (2014) Terrestrial Manual, Chapter 2.8.3. Paragraph 1.1.5. Paris, France.

[83] Giangaspero, M. (2013) Pestivirus Species Potential Adventitious Contaminants of Biological Products. Tropical Medicine \& Surgery, 1, 153.

https://doi.org/10.4172/2329-9088.1000153 
[84] Schirrmeier, H., Strebelow, G., Depner, K., Hoffmann, B. and Beer, M. (2004) Genetic and Antigenic Characterization of an Atypical Pestivirus Isolate, a Putative Member of a Novel Pestivirus Species. Journal of General Virology, 85, 3647-3652. https://doi.org/10.1099/vir.0.80238-0

[85] Decaro, N., Lucente, M.S., Mari, V., Cirone, F., Cordioli, P., et al. (2011) Atypical Pestivirus and Severe Respiratory Disease in Calves, Europe. Emerging Infectious Diseases, 17, 1549-1552. https://doi.org/10.3201/eid1708.101447

[86] Decaro, N., Lucente, M.S., Mari, V., Sciarretta, R., Pinto, P., et al. (2012) Hobi-Like Pestivirus in Aborted Bovine Fetuses. Journal of Clinical Microbiology, 50, 509-512. https://doi.org/10.1128/JCM.05887-11

[87] Decaro, N., Mari, V., Pinto, P., Lucente, M.S., Sciarretta, R., Cirone, F., Colaianni, M.L., Elia, G., Thiel, H.-J. and Buonavoglia, C. (2012) Hobi-Like Pestivirus: Both Biotypes Isolated from a Diseased Animal. Journal of General Virology, 93, 1976-1983. https://doi.org/10.1099/vir.0.044552-0

[88] Lanave, G., Decaro, N., Lucente, M.S., Guercio, A., Cavaliere, N., Purpari, G., Padalino, I., Larocca, V., Antoci, F., Marino, P.A., Buonavoglia, C. and Elia, G. (2017) Circulation of Multiple Subtypes of Bovine Viral Diarrhoea Virus Type 1 with No Evidence for HoBi-Like Pestivirus in Cattle Herds of Southern Italy. Infection, Genetics and Evolution, 50, 1-6. https://doi.org/10.1016/j.meegid.2017.02.009

[89] Giammarioli, M., Ceglie, L., Rossi, E., Bazzucchi, M., Casciari, C., Petrini, S. and De Mia, G.M. (2015) Increased Genetic Diversity of BVDV-1: Recent Findings and Implications Thereof. Virus Genes, 50, 147-151. https://doi.org/10.1007/s11262-014-1132-2

[90] Nagai, M., Ito, T., Sugita, S., Genno, A., Takeuchi, K., Ozawa, T., Sakoda, Y., Nishimori, T., Takamura, K. and Akashi, H. (2001) Genomic and Serological Diversity of Bovine Viral Diarrhea Virus in Japan. Archives of Virology, 146, 685-696. https://doi.org/10.1007/s007050170139

[91] Sato. A., Tateishi, K., Shinohara, M., Naoi, Y., Shiokawa, M., Aoki, H., Ohmori, K., Mizutani, T., Shirai, J. and Nagai, M. (2016) Complete Genome Sequencing of Bovine Viral Diarrhea Virus 1, Subgenotypes 1n and 1o. Genome Announcements, 4, e01744-15. https://doi.org/10.1128/genomeA.01744-15

[92] Biró, J., Oláh, P. and Palatka, Z. (1966) Attempt to Adapt the Lapinized Hog Cholera Virus Strain "Suvac" to Sheep. Acta Veterinaria Academiae Scientiarum Hungaricae, 16, 293-299.

[93] Center for Food Security and Public Health (2015) Classical Swine Fever. Factsheets. http://www.cfsph.iastate.edu/

[94] Loan, R.W. and Storm, M.M. (1968) Propagation and Transmission of Hog Cholera Virus in Non-Porcine Hosts. American Journal of Veterinary Research, 29, 807-811.

[95] Shimizu, M. and Kumagai, T. (1989) Experimental Infection of Pregnant Goats with Swine Fever Virus. Veterinary Microbiology, 20, 207-214. https://doi.org/10.1016/0378-1135(89)90044-8

[96] Mohamed, T.M. (2004) BVD Report UAE. https://www.researchgate.net/publication/236141896_BVD_ReportUAE2004

[97] Liu, Y., Yin, Z., Liu, S., Han, P., Hua, S., Yu, Y., Shi, Q., Ding, X., Liu, X. and Yu, R. (2003) Study on the Prevention of Bovine Viral Diarrhoea-Mucosal Disease of the Yak. Chinese Journal of Preventive Veterinary Medicine, No. 6.

[98] Cortez, A., Heinemann, M.B., de Castro, A.M.M.G., Soares, R.M., Pinto, A.M.V., Alfieri, A.A., Flores, E.F., Leite, R.C. and Richtzenhain, L.J. (2006) Genetic Charac- 
terization of Brazilian Bovine Viral Diarrhea Virus Isolates by Partial Nucleotide Sequencing of the 5'-UTR Region. Pesquisa Veterinária Brasileira, 26, 211-216. https://doi.org/10.1590/S0100-736X2006000400005

[99] Barros, S.C., Ramos, F., Paupério, S., Thompson, G. and Fevereiro, M. (2006) Phylogenetic Analysis of Portuguese Bovine Viral Diarrhoea Virus. Virus Research, 118, 192-195. https://doi.org/10.1016/j.virusres.2005.12.009

[100] Becher, P., Shannon, A.D., Tautz, N. and Thiel, H.-J. (1994) Molecular Characterization of Border Disease Virus, a Pestivirus from Sheep. Virology, 198, 542-551. https://doi.org/10.1006/viro.1994.1065

[101] Beer, M., Wolf, G. and Kaaden, O.R. (2002) Phylogenetic Analysis of the 5'-Untranslated Region of German BVDV Type II Isolates. Journal of Veterinary Medicine, Series B, 49, 43-47. https://doi.org/10.1046/j.1439-0450.2002.00536.x

[102] Caruso, C., Peletto, S., Cerutti, F., Modesto, P., Robetto, S., Domenis, L., Masoero, L. and Acutis, P.L. (2017) Evidence of Circulation of the Novel Border Disease Virus Genotype 8 in Chamois. Archives of Virology, 162, 511-515. https://doi.org/10.1007/s00705-016-3112-4

[103] Collett, M.S., Larson, R., Gold, C., Strick, D., Anderson, D.K. and Purchio, A.F. (1988) Molecular Cloning and Nucleotide Sequence of the Pestivirus Bovine Viral Diarrhea Virus. Virology, 165, 191-199. https://doi.org/10.1016/0042-6822(88)90672-1

[104] Couvreur, B., Letellier, C., Collard, A., Quenon, P., Dehan, P., Hamers, C., Pastoret, P.P. and Kerkhofs, P. (2002) Genetic and Antigenic Variability in Bovine Viral Diarrhea Virus (BVDV) Isolates from Belgium. Virus Research, 85, 17-28. https://doi.org/10.1016/S0168-1702(02)00014-X

[105] Decaro, N., Lucente, M.S., Lanave, G., Gargano, P., Larocca, V., Losurdo, M., Ciambrone, L., Marino, P.A., Parisi, A., Casalinuovo, F., Buonavoglia, C. and Elia, G. (2016) Evidence for Circulation of Bovine Viral Diarrhoea Virus Type 2c in Ruminants in Southern Italy. Transboundary and Emerging Diseases, 64, 1935-1944.

[106] De Moerlooze, L., Lecomte, C., Brown-Shimmer, S., Schmetz, D., Guiot, C., Vandenbergh, D., Allaer, D., Rossius, M., Chappuis, G., Dina, D., Renard, A. and Martial, J.A. (1993) Nucleotide Sequence of the Bovine Viral Diarrhoea Virus Osloss Strain: Comparison with Related Viruses and Identification of Specific DNA Probes in the 5' Untranslated Region. Journal of General Virology, 74, 1433-1438.

https://doi.org/10.1099/0022-1317-74-7-1433

[107] Deng, Y., Shan, T.L., Tong, W., Zheng, X.C., Guo, Y.Y., Zheng, H., Cao, S.J. and Wen, X.T. (2014) Genomic Characterization of a Bovine Viral Diarrhea Virus 1 Isolate from Swine. Archives of Virolog, 159, 2513-2517. https://doi.org/10.1007/s00705-014-2064-9

[108] Dubois, E., Russo, P., Prigent, M. and Thiéry, R. (2008) Genetic Characterization of Ovine Pestiviruses Isolated in France, between 1985 and 2006. Veterinary Microbiology, 130, 69-79. https://doi.org/10.1016/j.vetmic.2008.01.002

[109] Flores, E.F., Ridpath, J.F., Weiblen, R., Vogel, F.S.F. and Gil, L.H.V.G. (2002) Phylogenetic Analysis of Brazilian Bovine Viral Diarrhea Virus Type 2 (BVDV-2) Isolates: Evidence for a Subgenotype within BVDV-2. Virus Research, 87, 51-60. https://doi.org/10.1016/S0168-1702(02)00080-1

[110] Giammarioli, M., Pellegrini, C., Casciari, C., Rossi, E. and De Mia, G.M. (2008) Genetic Diversity of Bovine Viral Diarrhea Virus 1: Italian Isolates Clustered in at Least Seven Subgenotypes. Journal of Veterinary Diagnostic Investigation, 20, 783-788. https://doi.org/10.1177/104063870802000611 
[111] Giammarioli, M., Rossi, E., Casciari, C., Bazzucchi, M., Claudia, T. and De Mia, G.M. (2015) Genetic Characterization of Border Disease Virus (BDV) Isolates from Small Ruminants in Italy. Virus Genes, 50, 321-324. https://doi.org/10.1007/s11262-014-1165-6

[112] Giangaspero, M., Vacirca, G., Harasawa, R., Büttner, M., Panuccio, A., De Giuli Morghen, C., Zanetti, A., Belloli, A. and Verhulst, A. (2001) Genotypes of Pestivirus RNA Detected in Live Virus Vaccines for Human Use. The Journal of Veterinary Medical Science, 63, 723-733. https://doi.org/10.1292/jvms.63.723

[113] Gong, W., Zhang, L., Lu, Z., Jia, J., Wang, M., Peng, Z., Guo, H., Shi, J. and Tu, C. (2016) Complete Genome Sequence of a Novel Sub-Subgenotype 2.1g Isolate of Classical Swine Fever Virus from China. Archives of Virology, 161, 2613-2617. https://doi.org/10.1007/s00705-016-2932-6

[114] Grebennikova, T.V., Zaberezhnyi, A.D., Sergeev, V.A., Biketov, S.F., Aliper, T.I. and Nepoklonov, E.A. (1999) Genetic Characteristics of the KC Vaccine Strain of Hog Cholera Virus: Comparative Analysis of The primary Sequence of Surface Glycoprotein E(rns), E1, and E2 Genes. Molecular Genetics, Microbiology and Virology, 2, 34-40.

[115] Harasawa, R. (1995) Adventitious Pestivirus RNA in Live Virus Vaccines against Bovine and Swine Diseases. Vaccine, 13, 100-103. https://doi.org/10.1016/0264-410X(95)80018-9

[116] Harasawa, R., Hikiji, K., Tanabe, H., Takada, Y. and Mizusawa, H. (1993) Detection of Adventitious Pestivirus in Cell Cultures by Polymerase Chain Reaction Using Nested-Pair Primers. Tissue Culture Research Communications, 12, 215-220.

[117] Harasawa, R. and Tomiyama, T. (1994) Evidence of Pestivirus RNA in Human Virus Vaccines. Journal of Clinical Microbiology, 32, 1604-1605.

[118] Harasawa, R. and Sasaki, T. (1995) Sequence Analysis of the 5' Untranslated Region of Pestivirus RNA Demonstrated in Interferons for Human Use. Biologicals, 23, 263-269. https://doi.org/10.1006/biol.1995.0044

[119] Hofmann, M. and Bossy, S. (1998) Classical Swine Fever in 1993 in Switzerland: Molecular Epidemiologic Characterization of the Virus Isolate. Schweizer Archiv fur Tierheilkunde, 140, 365-370.

[120] Jiang, D.L., Liu, G.H., Gong, W.J., Li, R.C., Hu, Y.F., Tu, C. and Yu, X.L. (2013) Complete Genome Sequences of Classical Swine Fever Virus Isolates Belonging to a New Subgenotype, 2.1c, from Hunan Province, China. Genome Announcements, 1 , E00080-12. https://doi.org/10.1128/genomeA.00080-12

[121] Jones, L.R., Zandomeni, R.O. and Weber, E.L. (2001) Genetic Typing of Bovine Viral Diarrhea Virus Isolates from Argentina. Veterinary Microbiology, 81, 367-375. https://doi.org/10.1016/S0378-1135(01)00367-4

[122] Jones, L.R., Cigliano, M.M., Zandomeni, R.O. and Weber, E.L. (2004) Phylogenetic Analysis of Bovine Pestiviruses: Testing the Evolution of Clinical Symptoms. Cladistics, 20, 443-453. https://doi.org/10.1111/j.1096-0031.2004.00030.x

[123] Joo, S.K., Lim, S.I., Jeoung, H.Y., Song, J.Y., Oem, J.K., Mun, S.H. and An, D.J. (2013) Genome Sequence of Bovine Viral Diarrhea Virus Strain 10JJ-SKR, Belonging to Genotype 1d. Genome Announcements, 1, e00565-13. https://doi.org/10.1128/genomeA.00565-13

[124] Kamboj, A., Patel, C.L., Chaturvedi, V.K., Saini, M. and Gupta, P.K. (2014) Complete Genome Sequence of an Indian Field Isolate of Classical Swine Fever Virus Belonging to Subgenotype 1.1. Genome Announcements, 2, e00886-14.

[125] Kawanishi, N., Tsuduku, S., Shimizu, H., Ohtani, Y., Kameyama, K., Yamakawa, M., 
Tsutsui, T., Matsuura, K., Ohashi, S., Isobe, T. and Yamada, S. (2014) First Isolation of Border Disease Virus in Japan Is from a Pig Farm with No Ruminants. Veterinary Microbiology, 171, 210-214. https://doi.org/10.1016/j.vetmic.2014.03.032

[126] Leifer, I., Hoffmann, B., Hoper, D., Bruun Rasmussen, T., Blome, S., Strebelow, G., Horeth-Bontgen, D., Staubach, C. and Beer, M. (2010) Molecular Epidemiology of Current Classical Swine Fever Virus Isolates of Wild Boar in Germany. Journal of General Virology, 91, 2687-2697. https://doi.org/10.1099/vir.0.023200-0

[127] Li, X., Xu, Z., He, Y., Yao, Q., Zhang, K., Jin, M., Chen, H. and Qian, P. (2006) Genome Comparison of a Novel Classical Swine Fever Virus Isolated in China in 2004 with Other CSFV Strains. Virus Genes, 33, 133-142. https://doi.org/10.1007/s11262-005-0048-2

[128] Liu, H., Li, Y., Gao, M., Wen, K., Jia, Y., Liu, X., Zhang, W., Ma, B. and Wang, J. (2012) Complete Genome Sequence of a Bovine Viral Diarrhea Virus 2 from Commercial Fetal Bovine Serum. Journal of Virology, 86, 10233.

https://doi.org/10.1128/JVI.01581-12

[129] Liu, X., Mao, L., Li, W., Yang, L., Zhang, W., Wei, J. and Jiang, J. (2013) Genome Sequence of Border Disease Virus Strain JSLS12-01, Isolated from Sheep in China. Genome Announcements, 1, e00502-13. https://doi.org/10.1128/genomeA.00502-13

[130] Luzzago, C., Bandi, C., Bronzo, V., Ruffo, G. and Zecconi, A. (2001) Distribution Pattern of Bovine Viral Diarrhoea Virus Strains in Intensive Cattle Herds in Italy. Veterinary Microbiology, 83, 265-274. https://doi.org/10.1016/S0378-1135(01)00429-1

[131] Mishra, N., Pattnaik, B., Vilcek, S., Patil, S.S., Jain, P., Swamy, N., Bhatia, S. and Pradhan, H.K. (2004) Genetic Typing of Bovine Viral Diarrhoea Virus Isolates from India. Veterinary Microbiology, 104, 207-212.

https://doi.org/10.1016/j.vetmic.2004.08.003

[132] Meyers, G., Rümenapf, T. and Thiel, H.-J. (1989) Molecular Cloning and Nucleotide Sequence of the Genome of Hog Cholera Virus. Virology, 171, 555-567. https://doi.org/10.1016/0042-6822(89)90625-9

[133] Meyers, G., Sallmüller, A. and Büttner, M. (1999) Mutations Abrogating the Rnase Activity in Glycoprotein $\mathrm{E}^{\text {rns }}$ of the Pestivirus Classical Swine Fever Virus Lead to Virus Attenuation. Journal of Virology, 73, 10224-10235.

[134] Moormann, R.M.J., Warmerdam, P.A.M., van der Meer, B., Schaaper, W.M.M., Wensvoort, G. and Hulst, M.M. (1990) Molecular Cloning and Nucleotide Sequence of Hog Cholera Virus Strain Brescia and Mapping of Genomic Region Encoding Envelope Protein E1. Virology, 177, 184-198.

https://doi.org/10.1016/0042-6822(90)90472-4

[135] Moormann, R.M.J., van Gennip, H.G.P., Miedema, G.K.W., Hulst, M.M. and van Rijn, P.A. (1996) Infectious RNA Transcribed from an Engineered Full-Length cDNA Template of the Genome of a Pestivirus. Journal of Virology, 70, 763-770.

[136] Mosena, A.C.S., Cibulski, S.P., Weber, M.N., Silveira, S., Silva, M.S., Mayer, F.Q., Roehe, P.M. and Canal, C.W. (2017) Genomic and Antigenic Relationships between two "HoBi"-Like Strains and Other Members of the Pestivirus genus. Archives of Virology, 162, 3025-3034. https://doi.org/10.1007/s00705-017-3465-3

[137] Nagai, M., Aoki, H., Sakoda, Y., Kozasa, T., Tominaga-Teshima, K., Mine, J., Abe, Y., Tamura, T., Kobayashi, T., Nishine, K., Tateishi, K., Suzuki, Y., Fukuhara, M., Ohmori, K., Todaka, R., Katayama, K., Mizutani, T., Nakamura, S., Kida, H. and Shirai, J. (2014) Molecular, Biological, and Antigenic Characterization of a Border Disease Virus Isolated from a Pig During Classical Swine Fever Surveillance in Ja- 
pan. Journal of Veterinary Diagnostic Investigation, 26, 547-552.

https://doi.org/10.1177/1040638714541837

[138] Nagai, M., Sakoda, Y., Mori, M., Hayashi, M., Kida, H. and Akashi, H. (2003) Insertion of Cellular Sequence and RNA Recombination in the Structural Protein Coding Region of Cytopathogenic Bovine Viral Diarrhoea Virus. Journal of General Virology, 84, 447-452. https://doi.org/10.1099/vir.0.18773-0

[139] Nagai, M., Sato, M., Nagano, H., Pang, H., Kong, X., Murakami, T., Ozawa, T. and Akashi, H. (1998) Nucleotide Sequence Homology to Bovine Viral Diarrhea Virus 2 (BVDV 2) in the 5' Untranslated Region of BVDVs from Cattle with Mucosal Disease or Persistent Infection in Japan. Veterinary Microbiology, 60, 271-276. https://doi.org/10.1016/S0378-1135(98)00158-8

[140] Patil, S.S., Hemadri, D., Shankar, B.P., Raghavendra, A.G., Veeresh, H., Sindhoora, B., Chandan, S., Sreekala, K., Gajendragad, M.R. and Prabhudas, K. (2010) Genetic Typing of Recent Classical Swine Fever Isolates from India. Current Microbiology, 141, 367-373.

[141] Peletto, S., Caruso, C., Cerutti, F., Modesto, P., Zoppi, S., Dondo, A., Acutis, P.L. and Masoero, L. (2016) A New Genotype of Border Disease Virus with Implications for Molecular Diagnostics. Archives of Virology, 161, 471-477.

https://doi.org/10.1007/s00705-015-2696-4

[142] Peletto, S., Zuccon, F., Pitti, M., Gobbi, E., Marco, L.D., Caramelli, M., Masoero, L. and Acutis, P.L. (2012) Detection and Phylogenetic Analysis of an Atypical Pestivirus, Strain IZSPLV_To. Research in Veterinary Science, 92, 147-150. https://doi.org/10.1016/j.rvsc.2010.10.015

[143] Pellerin, C., Van den Hurk, J., Lecomte, J. and Tijssen, P. (1994) Identification of a New Group of Bovine Diarrhea Virus Strains Associated with Severe Outbreaks and High Mortalities. Virology, 203, 260-268. https://doi.org/10.1006/viro.1994.1483

[144] Pizarro Lucero, J., Celedón, M.O., Aguilera, M. and De Calisto, A. (2006) Molecular Characterization of Pestiviruses Isolated from Bovines in Chile. Veterinary Microbiology, 115, 208-217. https://doi.org/10.1016/j.vetmic.2006.02.009

[145] Rasmussen, T.B., Reimann, I., Uttenthal, A., Leifer, I., Depner, K., Schirrmeier, H. and Beer, M. (2010) Generation of Recombinant Pestiviruses Using a Full-Genome Amplification Strategy. Veterinary Microbiology, 142, 13-17. https://doi.org/10.1016/j.vetmic.2009.09.037

[146] Ridpath, J.F. and Bolin, S.R. (1997) Comparison of the Complete Genomic Sequence of the Border Disease Virus, BD31, to Other Pestiviruses. Virus Research, 50, 237-243. https://doi.org/10.1016/S0168-1702(97)00064-6

[147] Ridpath, J.F., Bolin, S.R. and Dubovi, E.J. (1994) Segregation of Bovine Viral Diarrhoea Virus into Genotypes. Virology, 205, 66-74.

https://doi.org/10.1006/viro.1994.1620

[148] Risatti, G.R., Borca, M.V., Kutish, G.F., Lu, Z., Holinka, L.G., French, R.A., Tulman, E.R. and Rock, D.L. (2005) The E2 Glycoprotein of Classical Swine Fever Virus Is a Virulence Determinant in Swine. Journal of Virology, 79, 3787-3796. https://doi.org/10.1128/JVI.79.6.3787-3796.2005

[149] Ruggli, N., Moser, C., Mitchell, D., Hofmann, M. and Tratschin, J.D. (1995) Baculovirus Expression and Affinity Purification of Protein E2 of Classical Swine Fever Virus Strain Alfort/187. Virus Genes, 10, 115-126. https://doi.org/10.1007/BF01702592

[150] Shen, H., Pei, J., Bai, J., Zhao, M., Ju, C., Yi, L., Kang, Y., Zhang, X., Chen, L., Li, Y., Wang, J. and Chen, J. (2011) Genetic Diversity and Positive Selection Analysis of 
Classical Swine Fever Virus Isolates in South China. Virus Genes, 43, 234-242. https://doi.org/10.1007/s11262-011-0625-5

[151] Stadejek, T., Warg, J. and Ridpath, J.F. (1996) Comparative Sequence Analysis of the 5' Noncoding Region of Classical Swine Fever Virus Strains from Europe, Asia, and America. Archives of Virology, 141, 771-777. https://doi.org/10.1007/BF01718335

[152] Stalder, H.P., Meier, Ph., Pfaffen, G., Wageck-Canal, C., Rüfenacht, J., Schaller, P., Bachofen, C., Marti, S., Vogt, H.R. and Peterhans, E. (2005) Genetic Heterogeneity of Pestiviruses of Ruminants in Switzerland. Preventive Veterinary Medicine, 72, 37-41. https://doi.org/10.1016/j.prevetmed.2005.01.020

[153] Sullivan, D.G., Chang, G.-J., Trent, D.W. and Akkina, R.K. (1994) Nucleotide Sequence Analysis of the Structural Gene Coding Region of the Pestivirus Border Disease Virus. Virus Research, 33, 219-228.

https://doi.org/10.1016/0168-1702(94)90104-X

[154] Tajima, M., Frey, H.R., Yamato, O., Maede, Y., Moenning, V., Scholz, H. and Greiser-Wilke, I. (2001) Prevalence of Genotypes 1 and 2 of Bovine Viral Diarrhea Virus in Lower Saxony, Germany. Virus Research, 76, 31-42.

https://doi.org/10.1016/S0168-1702(01)00244-1

[155] Tao, J., Wang, Y., Wang, J., Wang, J.Y. and Zhu, G.Q. (2013) Identification and Genetic Characterization of New Bovine Viral Diarrhea Virus Genotype 2 Strains in Pigs Isolated in China. Virus Genes, 46, 81-87. https://doi.org/10.1007/s11262-012-0837-3

[156] Thabti, F., Letellier, C., Hammami, S., Pepin, M., Ribière, M., Mesplède, A., Kerkhofs, P. and Russo, P. (2005) Detection of a Novel Border Disease Virus Subgroup in Tunisian Sheep. Archives of Virology, 150, 215-229. https://doi.org/10.1007/s00705-004-0427-3

[157] Tomar, N., Gupta, A., Arya, R.S., Somvanshi, R., Sharma, V. and Saikumar, G. (2015) Genome Sequence of Classical Swine Fever Virus Genotype 1.1 with a Genetic Marker of Attenuation Detected in a Continuous Porcine Cell Line. Genome Announcements, 3, e00375-15. https://doi.org/10.1128/genomeA.00375-15

[158] Topliff, C.L. and Kelling, C.L. (1998) Virulence Markers in the 5' Untranslated Region of Genotype 2 Bovine Viral Diarrhea Virus Isolates. Virology, 250, 164-172. https://doi.org/10.1006/viro.1998.9350

[159] Uttenthal, A., Le Potier, M.F., Romero, L., De Mia, G.M. and Floegel-Niesmann, G. (2001) Classical Swine Fever (CSF) Trial I. Challenge Marker Vaccine Studies in Weaner Pigs. Veterinary Microbiology, 83, 85-106. https://doi.org/10.1016/S0378-1135(01)00409-6

[160] Valdazo-González, B., Alvarez-Martínez, M. and Greiser-Wilke, I. (2006) Genetic Typing and Prevalence of Border Disease Virus (BDV) in Small Ruminant Flocks in Spain. Veterinary Microbiology, 117, 141-153. https://doi.org/10.1016/j.vetmic.2006.06.008

[161] Valdazo-González, B., Alvarez-Martínez, M. and Sandvik, T. (2007) Genetic and Antigenic Typing of Border Disease Virus Isolates in Sheep from the Iberian Peninsula. The Veterinary Journal, 174, 316-324. https://doi.org/10.1016/j.tvjl.2006.10.002

[162] Vilček, O.E., Durkovic, B., Bobakova, M., Sharp, G. and Paton, D.J. (2002) Identification of Bovine Viral Diarrhoea Virus 2 in Cattle in Slovakia. Veterinary Record, 151, 150-152. https://doi.org/10.1136/vr.151.5.150

[163] Vilček, S., Greiser-Wilke, I., Durkovic, B., Obritzhauser, W., Deutz, A. and Kofer, J. 
(2003) Genetic Diversity of Recent Bovine Viral Diarrhoea Viruses from the Southeast of Austria (Styria). Veterinary Microbiology, 91, 285-291. https://doi.org/10.1016/S0378-1135(02)00296-1

[164] Vilček, S., Nettleton, P.F., Paton, D.J. and Belák, S. (1997) Molecular Characterization of Ovine Pestiviruses. Journal of General Virology, 78, 725-735. https://doi.org/10.1099/0022-1317-78-4-725

[165] Vilcek, S., Paton, D.J., Durkovic, B., Strojny, L., Ibata, G., Moussa, A., Loitsch, A., Rossmanith, W., Vega, S., Scicluna, M. and Palfi, V. (2001) Bovine Viral Diarrhoea Virus Genotype 1 Can Be Separated into at Least Eleven Genetic Groups. Archives of Virology, 146, 99-115. https://doi.org/10.1007/s007050170194

[166] Widjojoatmodjo, M.N., van Gennip, H.G., de Smit, A.J. and Moormann, R.J. (1999) Comparative Sequence Analysis of Classical Swine Fever Virus Isolates from the Epizootic in The Netherlands in 1997-1998. Veterinary Microbiology, 66, 291-299. https://doi.org/10.1016/S0378-1135(99)00017-6

[167] Xia, H., Larska, M., Giammarioli, M., De Mia, G.M., Cardeti, G., Zhou, W., Alenius, S., Belak, S. and Liu, L. (2013) Genetic Detection and Characterization of Atypical Bovine Pestiviruses in Foetal Bovine Sera Claimed to Be of Australian Origin. Transboundary and Emerging Diseases, 60, 284-288. https://doi.org/10.1111/j.1865-1682.2012.01341.x

[168] Yamamoto, T., Kozasa, T., Aoki, H., Sekiguchi, H., Morino, S. and Nakamura, S. (2008) Genomic Analyses of Bovine Viral Diarrhea Viruses Isolated from Cattle Imported into Japan between 1991 and 2005. Veterinary Microbiology, 127, 386-391. https://doi.org/10.1016/j.vetmic.2007.08.020

[169] Zhou, W., Gao, S., Podgorska, K., Stadejek, T., Qiu, H.J., Yin, H., Drew, T. and Liu, L. (2014) Rovac Is the Possible Ancestor of the Russian Lapinized Vaccines LK-VNIVViM and CS Strains but Not the Chinese Strain (C-Strain) Vaccine against Classical Swine Fever. Vaccine, 32, 6639-6642.

https://doi.org/10.1016/j.vaccine.2014.09.058 